\title{
"ENVIRONMENTALLY FRIENDLY" RENORMALIZATION
}

\author{
Denjoe O' Connor \\ School of Theoretical Physics, \\ Dublin Institute for Advanced Studies, \\ 10 Burlington Road, \\ Dublin 4, Ireland. \\ C.R. Stephens \\ Institute for Theoretical Physics, \\ Rijksuniversiteit Utrecht, \\ Princetonplein 5, \\ 3508TA Utrecht, Netherlands.
}

\begin{abstract}
We analyze the renormalization of systems whose effective degrees of freedom are described in terms of fluctuations which are "environment" dependent. Relevant environmental parameters considered are: temperature, system size, boundary conditions, and external fields. The points in the space of "coupling constants" at which such systems exhibit scale invariance coincide only with the fixed points of a global renormalization group which is necessarily environment dependent. Using such a renormalization group we give formal expressions to two loops for effective critical exponents for a generic crossover induced by a relevant mass scale $g$. These effective exponents are seen to obey scaling laws across the entire crossover, including hyperscaling, but in terms of an effective dimensionality, $d_{\text {eff }}=4-\gamma_{\lambda}$, which represents the effects of the leading irrelevant operator. We analyze the crossover of an $O(N)$ model on a $d$ dimensional layered geometry with periodic, antiperiodic and Dirichlet boundary conditions. Explicit results to two loops for effective exponents are obtained using a [2,1] Padé resummed coupling, for: the "Gaussian model" $(N=-2)$, spherical model $(N=\infty)$, Ising Model $(N=1)$, polymers $(N=0)$, XY-model $(N=2)$ and Heisenberg $(N=3)$ models in four dimensions. We also give two loop Padé resummed results for a three dimensional Ising ferromagnet in a transverse magnetic field and corresponding one loop results for the two dimensional model. One loop results are also presented for a three dimensional layered Ising model with Dirichlet and antiperiodic boundary conditions. Asymptotically the effective exponents are in excellent agreement with known results.
\end{abstract}




\section{$\S$ 1. INTRODUCTION}

Field theory is our most powerful tool for describing systems with a large number of degrees of freedom, and in particular in situations where the latter can act collectively. In one guise or another such systems comprise a large proportion of current physics. Even though one starts at a certain energy scale with a "fundamental" set of degrees of freedom, which may be very elementary looking, e.g. QED, one knows that as one progresses to different (usually lower) energy scales, the original elementary degrees of freedom, though in principle offering a complete description of the low energy physics, become, in practice, unusable.

For example, one would be rather perverse to try and describe a phase transition in a ferromagnet in terms of QED. As is well known the transition is much better described in terms of a Landau-Ginzburg-Wilson Hamiltonian (LGW Hamiltonian) based on effective degrees of freedom associated with a magnetization density $\phi(x)$. This is an implementation of the philosophy of effective field theory, whereby the theory provides a good description only at energy scales where the effective degrees of freedom chosen offer a reasonably faithful representation of the physics. For instance, if we heated up the ferromagnet until it became a plasma of nuclei and electrons, then a description in terms of a LGW effective Hamiltonian would be rather inappropriate. The difficulty, of course, is that the effective degrees of freedom of the system are scale dependent, i.e. there is a "crossover" in them, and consequently in the physical behaviour of the system. Thus, if one requires a complete description of the physics at all scales one must be able to account for this fact. Our aim in this paper will be to develop further a formalism for describing such systems using field theoretic renormalization groups (RG). We will for the most part keep the formalism as general as possible, specializing in the latter half of the paper to specific crossovers of interest. Our reason for doing this is that the formalism is applicable to a very wide class of crossovers. By presenting the general results the reader is then at liberty to treat a crossover of particular interest to him/her merely by inserting into the appropriate parts of the formalism the particular details specific to that crossover. We will use a language that is hopefully accessible to people with some field theoretic background in both particle physics and statistical physics as the applications of the formalism are equally applicable to both areas.

To illustrate the ubiquity of the concept of crossover behaviour we will briefly mention (without detailed explanation) some physically pertinent examples. In QCD, at high energies, a description in terms of quark-gluon degrees of freedom is appropriate, whilst at low energies baryon-meson degrees of freedom are more suitable. In four dimensional finite temperature field theory, for $T \gg m(T)$, where $m(T)$ is a typical finite temperature mass scale, three dimensional degrees of freedom are appropriate, whereas for $T \ll m(T)$ they are four dimensional. In the early universe the effective degrees of freedom are time dependent due to the cosmological expansion. In fact, for quantum field theory in curved spacetime where one is concerned with "in" and "out" vacua, the difference between these vacua, which results in particle production, can be thought of as being representative of the difference between "in" and "out" effective degrees of freedom. In critical phenomena crossover can be induced by many different effects. One exciting much interest currently is the effect of randomness, e.g. a random magnetic field, random impurities etc. In such cases the effective degrees of freedom depend on the concentration of the impurities or the strength of the magnetic field. Another eliciting great interest concerns the effects of finite size. This is of relevance in lattice simulations and also in real experimental systems.

The physics of crossovers then, concerns systems which exhibit qualitatively different degrees of freedom at different scales. Our goal is to qualitatively and quantitatively describe such systems. For an exact model of course there is no problem, however, even 
though one has an exact answer that does not necessarily imply that one has a good intuitive understanding of the physics. Often with an exact model one can not see the wood from the trees. Generically one must resort to approximation techniques. The dominant one, of course, is perturbation theory, where one must answer the crucial question: what parameter should one perturb in? Often the difference between getting a nonsensical versus a reliable answer comes down to being able to pick and work with a good expansion parameter.

In crossover problems one starts with some interacting Hamiltonian which will come with a coupling constant, $\lambda$, which one would be tempted to use as an expansion parameter when computing the correlation functions associated with this Hamiltonian. However, the starting "bare" (microscopic) Hamiltonian will only offer an accurate perturbative description when one looks at scales $\kappa \sim \Lambda, \Lambda$ being the UV cutoff (inverse lattice spacing) for the theory. At scales $\kappa \ll \Lambda$, as is well known, the bare parameters offer a perturbatively useless description. One must therefore perform a renormalization to a new set of parameters. This is done by defining correlation functions at a fiducial scale $\kappa^{\prime}$, and using them as the parameters with respect to which one describes physics at a scale $\kappa \neq \kappa^{\prime}$. As long as the two scales $\kappa$ and $\kappa^{\prime}$ are not too different, the description of physics at the scale $\kappa$ in terms of parameters defined at the scale $\kappa^{\prime}$ will be perturbatively reliable. The crossover from bare degrees of freedom to renormalized ones, associated with taking the continuum limit or the removal of a regularizing UV cutoff, is perhaps the most elementary example of a crossover. In contrast to a scale invariant system where the bare degrees of freedom and the renormalized ones are relatively simply related they are quite different when the system is not scale invariant. In the presence of a cutoff the bare and renormalized degrees of freedom are always different as the system cannot be scale invariant.

So why are the bare and renormalized effective degrees of freedom so different? Due to the effects of fluctuations; the fluctuations "dress" the bare parameters. This dressing in principle can become infinite if one takes the $\Lambda \rightarrow \infty$ limit. For us the crucial difference is between a large dressing and a small dressing not between an infinite one and a finite one. Moreover, as far as the RG is concerned, we feel that there is no particular virtue in emphasizing UV dressings as opposed to IR ones. In particle physics it is the former which have received most attention, however, the machinery of the RG must be invoked any time one has large dressings of one's parameters, irrespective of from which particular end of the spectrum the dominant fluctuations arise. Large dressings imply that one is not tracking the effective degrees of freedom in the problem very well. Renormalization is a methodology by which one can use the freedom to reparametrize one's theory to find parameters which track the effective degrees of freedom more accurately. In the case of bare versus renormalized parameters, without interactions the bare parameters cannot get dressed and remain the same. Indeed, it is the presence of interactions that induces the crossover between them. The renormalization carried out was dependent on the interaction strength $\lambda$. That is why the renormalization worked in the first place, because it depended on the parameter that was inducing the crossover. We could have been perverse and tried to renormalize the sytem in a $\lambda$ independent way. The Gaussian theory would have a large dressing associated with the specific heat, or vacuum energy, for instance. This would have to be renormalized. After the renormalizations associated with the Gaussian theory were performed one would still find that connected correlation functions had large dressings. This is a reflection of the fact that the renormalization process, using only counterterms appropriate for the Gaussian theory, was not sufficient to make sense of the interacting theory.

Generically, in the renormalization process one takes fluctuations between scales $\Lambda$ and $\kappa^{\prime}$, contributing to a given correlation function, and absorbs them into a redefinition of a parameter, which is usually a vertex function at the scale $\kappa^{\prime}$. To investigate physics 
at a scale $\kappa$, one considers the fluctuations between $\kappa$ and $\kappa^{\prime}$, explicitly in perturbation theory, in terms of the coupling $\lambda$ defined at the scale $\kappa^{\prime}$ instead of the bare coupling. The important question of course is what fluctuations are being absorbed into redefinitions of the parameters? If we renormalized using only counterterms associated with the Gaussian theory then only non-interacting fluctuations are being absorbed into redefinitions. For the connected correlation functions this is equivalent to not having absorbed in anything. Obviously this won't help much. In the standard $\lambda$ dependent case all the fluctuations associated with interacting effective degrees of freedom between the scales $\Lambda$ and $\kappa^{\prime}$ have been absorbed. What we do know for a fact is that the effective degrees of freedom in the system are interacting, therefore one would expect to get more sense out of a renormalization procedure that explicitly takes account of this fact. The reader might wonder why we have gone to such lengths analyzing such a standard procedure, and why on earth one would ever try to renormalize an interacting theory using renormalization that was explicitly $\lambda$ independent. We hope by the end of the paper the reason will be clear. For the moment we will simply say that a successful renormalization is one that can take proper account of what the true effective degrees of freedom of a system are. In the above they are $\lambda$ dependent, therefore a good renormalization should be $\lambda$ dependent.

So, an important aspect of renormalization is that it is a methodology whereby through a suitable redefinition of parameters one can make perturbative sense out of something that originally was perturbative nonsense. How sensible the expansion actually becomes, however, is crucially dependent on what physics the renormalization process can capture. In the above the renormalization had to capture the fact that the effective degrees of freedom were interacting. If one considered an interacting field theory in a three dimensional box of size $L$, one could renormalize the theory in an $L$ independent fashion. When one considered physics on scales $\kappa \sim L^{-1}$ one would find that the theory was perturbatively ill defined, whereas an appropriate $L$ dependent renormalization made perturbative sense. The reason for this, of course, is that the effective degrees of freedom in the system are explicitly $L$ dependent. An $L$ independent renormalization ignores this important physical fact. The only fluctuations being absorbed into the renormalized parameters in this case are $L$ independent, no matter what renormalization scale one chooses. $L$ here is the parameter which induces the crossover and therefore a good renormalization scheme should be $L$ dependent.

More generally we will speak of a crossover being induced by a particular "environmental" variable. The idea to understand here is that the fluctuations in a system "feel" out the environment they are in. For instance in the above case of physics in a box, the size of the box $L$ is a relevant environmental variable, as clearly effective degrees of freedom associated with length scales $\ll L$ and $\sim L$ are completely different. The effective degrees of freedom are thus sensitive to the environment. There are many, many different environmental parameters describing all sorts of crossover systems. In this paper we will consider the effects of a generic environmental variable $g$, which could represent variously: finite size, temperature, spin anisotropy, long range interactions, cosmological constant, Hubble parameter, magnetization, electric/magnetic field and so on. What we will be showing is how to describe qualitatively and quantitatively the response of a system to changes in the environment. We will show that in order to obtain a perturbative description of such systems one must implement an RG which is explicitly dependent on the environment. We will call such RGs "environmentally friendly". If an environment "unfriendly", that is to say, environment independent, RG is implemented, we will see that this generically leads to breakdown of perturbation theory, wherein the theory becomes strongly coupled in terms of the effective degrees of freedom characteristic of the environmentally unfriendly $\mathrm{RG}$, and that large perturbative dressings appear which can actually become divergent. 
Crossover behaviour, as mentioned, is quite ubiquitous. It is not our intention here to give a review of what has been done, consequently we will be rather selective in our comments, and, more apologetically, in our references. In critical phenomena it has recieved much more attention than in particle physics (for an early review see [1]), though many problems in particle physics are essentially crossover problems. In principle, basically any laboratory system will exhibit crossover behaviour in some regime. Some of the more experimentally accessible ones are: uniaxial dipolar ferromagnets [2], systems exhibiting a bicritical point [3], dimensional crossover in liquid $\mathrm{He}^{4}$ [4], quantum ferromagnets ([5] and references therein), bulk/surface crossovers [6]. Dimensional crossover, one of the chief concerns of this paper, has been studied mainly in the context of finite size scaling [7]. Much work has been done on the latter, and finite size effects in general, in the context of lattice simulations; quantum Monte Carlo methods [8] are also closely related.

From a more theoretical standpoint, and more particularly from the point of view of RG theory, much work has also been done. For a typical crossover induced by a generic anisotropy parameter $g$, scaling formulations have been almost invariably based on RG's that are $g$ independent [9]. This automatically leads to critical and crossover exponents that are defined with respect to the isotropic $g=0$ fixed point. Such RG's would be incapable, in and of themselves, of bridging the crossover to the anisotropic $g=\infty$ fixed point. What we mean by this is that the fixed points of such RG's will not yield all the possible points of scale invariance of the system, in particular the anisotropic scale invariant system will be inaccessible. The fundamental length scale utilized is $\xi_{0}$, the correlation length of the isotropic system. It is pertinent to note that this is not the physical correlation length in the crossover system. The desire to make accessible another fixed point besides the isotropic one has often entailed the matching of asymptotic expansions around the anisotropic and isotropic fixed points, or the use of high temperature expansions in conjunction with an ansatz for the scaling function [10] [11]. Exact models have also played a role, for instance, the two dimensional Ising model [12] and the spherical model [13] in the context of finite size scaling.

An RG approach, due to Riedel and Wegner [14], and used by others, utilizes "model recursion relations". Here it is assumed known what the two fixed points of the crossover are, subsequently RG equations for the scaling fields are postulated that interpolate between the two fixed points. As there is no reference to the underlying microscopic Hamiltonian such a ploy can only offer qualitative information. In addition there are many different crossover systems that exhibit crossover between exactly the same two fixed points. Universality implies that two systems that lie in the same universality class can via a suitable rescaling of variables be shown to have exactly the same IR properties, e.g. a simple fluid and an Ising ferromagnet. For crossovers the asymptotic fixed points do not determine "crossover universality classes". The scaling functions associated with different crossovers between the same two fixed points cannot generally be transformed into one another by a change of scaling variable. However, there is a concept of two systems being in the same crossover universality class - a $(d-1)$-dimensional Ising model in a transverse magnetic field and a $d$-dimensional layered Ising model with periodic boundary conditions being a case in point. Wilsonian RGs of the "momentum shell integration" type have been frequently employed with varying success. These methods generally have two main defects: ease of extension beyond first order, and using too rough an approximation in the shell integration. Given that many physically different systems can crossover between the same asymptotic fixed points it is important to be able to pick out the details of the crossover curves in order to distinguish between them. By using an approximation on the momentum shell integration it is easy to blur such distinctions.

From a field theory point of view much less work has been done. This is mainly because 
of the preoccupation with renormalization in the context of UV divergences, though there are one or two notable exceptions. Amit and Goldschmidt [15] introduced the concept of generalized minimal subtraction (GMS) in the context of crossover at a bicritical point. Their results for $\gamma_{e f f}$, however, differ significantly from those found by Nelson and Domany [16] and independently by Seglar and Fisher [17] using momentum shell integration, in particular in that the latter find a characteristic "dip" in the curves. In this case the results of our methodology applied to a bicritical crossover [18] agree with the latter. We believe this to be due to a deficiency of GMS which fails to capture the true behaviour of the leading irrelevant operator which in our crossover formalism plays a crucial role. GMS was also applied to uniaxial dipolar ferromagnets in [19], once again the results of our analysis [20][18] are somewhat different. Schmeltzer [21] calculated $\gamma_{e f f}$ to one loop for three dimensional quantum ferroelectrics. Lawrie [22] considered dimensional crossover for $d$-dimensional quantal and $d+1$ dimensional finite-sized Ising models for $3<d<4$. Unlike our methods the $\varepsilon$ expansion methodology he used could not capture the crossover between two non-trivial fixed points as such an expansion is around the upper critical dimension which changes across the crossover. Nemirovsky and Freed ([23] and references therein) used minimal subtraction techniques but failed to be able to access the full crossover. Field theoretic results for dimensional crossover in a fully finite geometry or a cylinder have been obtained [24] but the techniques used have not been extended to the case of a system with more than one fixed point. In a particle physics context some interesting work has been done in the context of the finite temperature RG [25] (see [26] for some recent applications of our work to finite temperature field theory).

In this paper we will present a field theoretic formalism which is applicable to a very wide class of crossovers associated with a field theory in a particular environment. We have previously presented some one loop results in the case of dimensional crossover, where the environmental variable was $L$, the finite size of the system, both in the case of above [27] [28] and below [29] the critical point. In the latter the background magnetization was also a relevant environmental variable inducing a crossover. We have also considered, at one loop, uniaxial dipolar ferromagnets and bicritical systems in [20] and [18], where the environmental variables were $\alpha_{0}$ and $m$ respectively. Here $\alpha_{0}$ is the dipole-dipole coupling strength and $m$ is the mass of the $O(N-M)$ components for the case $O(N) \rightarrow O(M)$. The main thrust of the present paper is to generalize the techniques used to a generic environment induced crossover, and in particular, to extend the methods beyond one loop as there are many subtleties involved in doing so, not least of which is the application of resummation techniques. We present two loop expressions for effective exponents and some scaling functions, which are set up in just such a fashion that the reader interested in a specific crossover, may take them, insert the relevant environment dependence in the Feynman diagrams, crank the handle (analytically if possible, numerically if not), and spit out the answers. To explicitly demonstrate the efficacy of the methodology we consider dimensional crossover for a $d$ dimensional $O(N)$ model on a layered geometry with periodic, Dirichlet or antiperiodic boundary conditions. We give explicit, surprisingly simple, expressions for two loop effective exponents for a four dimensional layered system with periodic boundary conditions, and corresponding (not so simple) expressions for a three dimensional layered system. We also give results for Dirichlet and periodic boundary conditions. Two loop results for the effective exponents of a three dimensional Ising model in a transverse magnetic field are presented, as are one loop results for the corresponding two dimensional model. Graphical displays of our results can be found throughout the paper.

The format of the paper will be as follows: in section 2 we consider some formal aspects of renormalization, mainly to set notation. In section 3 we consider the relationship between the RG and the environment. Specifically, we compare and contrast the field 
theoretic and "Wilsonian" RGs asking and answering the question of what does one require of a "good" RG, and in particular addressing which RGs will access all the points of scale invariance of a physical system. We illustrate these ideas by comparing massless versus massive renormalization showing that the former yields an RG which has fewer fixed points than the latter. We discuss precisely why a good RG should be environmentally friendly. In sections 4 we derive formal two loop expressions for the Wilson functions of our generic $g$ dependent crossover. In section 5 we discuss further the idea of an environmentally friendly versus environmentally unfriendly RG, showing that the former offers a more global description of the crossover sytem, the latter being incapable of accessing some of the points of scale invariance of the system. We also discuss an RG where the environment itself "runs", which is useful for addressing questions such as: what is the shift in critical temperature due to changes in the environment? In section 6 we consider the consequences of an environmentally friendly RG, defining effective critical exponents and showing that they obey scaling laws, including effective hyperscaling with respect to an effective dimensionality which is a measure of the importance of the leading irrelevant operator across the crossover. We exhibit formal scaling forms for the crossover equation of state and crossover coexistence curve in terms of effective exponents. We exhibit nonlinear scaling fields which interpolate across the crossover becoming at the endpoints of the crossover the associated linear scaling fields. We discuss the concepts of crossover universality and crossover universality class. In section 7 we address the important question of "what should we perturb in?" We argue that an appropriate perturbation parameter is the resummed solution of the $\beta$ function of the environmentally friendly RG. We emphasize that in the context of the RG, perturbation theory is carried out at the level of the Wilson functions, all of which as perturbative expansions should be resummed. We resum these series using a [2,1] Padé approximant which is appropriate at the two loop level. When the Wilson functions are integrated (exponentiated) the resulting functions should not then be themselves expanded. We show that an environmentally friendly RG is most potent when all environment dependent fluctuations are absorbed into reparametrizations of the parameters. In section 8 we discuss the floating fixed point which yields a good approximation to crossovers via the solution of the crossover $\beta$ function as an algebraic equation instead of as a differential equation. In section 9 we present two loop diagramatic representations for effective exponents and scaling fields. These can be treated as "black box" type expressions where one inserts the particular environmental dependence in the Feynman diagrams and reads off the corresponding exponent. In section 10 we consider one and two loop results for an $O(N)$ model on a layered geometry with periodic boundary conditions paying special attention to some models of interest. We also discuss the effects of boundary conditions on the crossover. In section 11 we consider the quantum/classical crossover in a $d$ dimensional Ising model in a transverse magnetic field. We present both one and two loop results. In section 12 we draw some conclusions and make some speculations. There are two appendices containing various expressions for diagrams needed in the calculations.

\section{$\S$ 2. FORMAL RENORMALIZATION}

In this section we present a formal approach to the problem of renormalization. We begin with the action (LGW Hamiltonian)

$$
S\left[\varphi_{B}\right]=\int d^{d} x\left[\frac{1}{2}\left(\partial \varphi_{B}\right)^{2}+\frac{1}{2} m_{B}^{2} \varphi_{B}^{2}+\frac{1}{2} t_{B}(x) \varphi_{B}^{2}+\frac{\lambda_{B}}{4 !} \varphi_{B}^{4}-H_{B}(x) \varphi_{B}+g_{B} O_{B}\right]
$$

which represents the "microscopic" theory. The term $g_{B} O_{B}$ is used to abstractly refer to an "anisotropy" in the system, $O_{B}$ being an operator conjugate to the coupling $g_{B}$. We 
will think of $g_{B}=0$ as representing an isotropic system. Physically this anisotropy could be of diverse origin. The general methodology espoused in this paper is not confined to sytems described by the Hamiltonian (2.1), though the latter is general enough for most of our considerations. From time to time, however, we will extend our attention to a general Hamiltonian who's parameters live on an abstract "space of coupling constants" $\mathcal{M}$. Each of the parameters in (2.1) is associated with a coordinate direction in this space. We will for convenience tend to use the terminology associated with viewing (2.1) as the Hamiltonian of a magnetic system.

The partition function $Z$ is defined by the functional integral

$$
Z=e^{-W\left[J_{B}+H_{B}, t_{B}+m_{B}^{2}\right]}=\int[d \varphi] e^{-S\left[\varphi_{B}\right]+\int d^{d} x J_{B}(x) \varphi_{B}(x)}
$$

where $W$ is the generating functional of connected Greens functions (free energy for prescribed sources). $J_{B}$ is to be understood here as some formal source, differentiation with respect to which generates the connected Greens functions (correlation functions), and which will after generation of the correlation functions be put to zero. $H_{B}$ will represent a real magnetic field in the critical phenomena context. The effective action (free energy for prescribed magnetization), $\Gamma\left[\phi_{B}, t_{B}+m_{B}^{2}\right]$, is the generating functional of one particle irreducible $N$-point vertex functions and is given by the Legendre transform

$$
\Gamma\left[\phi_{B}, t_{B}+m_{B}^{2}\right]=W\left[J_{B}+H_{B}, t_{B}\right]+\int d^{d} x\left(J_{B}(x)+H_{B}(x)\right) \phi_{B}(x)
$$

where $\phi_{B}=<\varphi_{B}>_{J, H}=\bar{\phi}_{B}+<\varphi_{B}>_{J}, \bar{\phi}_{B}$ being the magnetization when $J=0$. The vertex functions are then defined in terms of functional derivatives of $\Gamma\left[\phi_{B}, t_{B}+m_{B}^{2}\right]$ with respect to $\phi_{B}$ and $t_{B}$. Specifically

$$
\begin{gathered}
\Gamma\left[\phi_{B}, t_{B}+m_{B}^{2}\right]=\sum_{N=0}^{\infty} \frac{1}{N !} \Gamma_{B}^{(N)}\left(\bar{\phi}_{B}, t_{B}+m_{B}^{2}\right)\left(\phi_{B}-\bar{\phi}_{B}\right)^{N} \\
\Gamma_{B}^{(N)}\left(\bar{\phi}_{B}, t_{B}+m_{B}^{2}\right)=\sum_{M=0}^{\infty} \frac{1}{M !} \Gamma_{B}^{(N, M)}\left(\bar{\phi}_{B}, m_{B}^{2}\right) t_{B}^{M}
\end{gathered}
$$

where implicit multiple integrations over position or momenta are understood.

Note that $\bar{\phi}_{B}=0$ above the critical temperature when $H_{B}=0$, and hence the quantities $\Gamma^{(N)}$ are independent of $\bar{\phi}_{B}$ in this domain. For $H_{B} \neq 0$, or below the critical temperature, there is a non-zero spontaneous magnetization $\bar{\phi}_{B}$. Deviations from this magnetization are responses to the externally applied source $J_{B}$, they again will be set to zero once the vertex functions are obtained. The equation of state is given by

$$
\Gamma_{B}^{(1)}=H_{B}
$$

when $J_{B}=0$. This is the basic equation of the effective action and serves to determine the background magnetization $\bar{\phi}_{B}$.

The calculation of correlation functions more often than not requires the introduction of an ultraviolet (UV) cutoff (physical or otherwise). An infrared (IR) cutoff is frequently also 
necessary. We renormalize by imposing normalization conditions, though for the moment we will be somewhat nebulous about the precise normalization point. Two quantities required in this procedure are the wave function renormalization constant $Z_{\phi}$, where $\phi=$

$Z_{\phi}^{-\frac{1}{2}} \phi_{B}$, and the composite operator renormalization constant $Z_{\phi^{2}}$. The relation between the bare and renormalized vertex functions is

$$
\Gamma_{B}^{(N, M)}=Z_{\phi}^{-\frac{N}{2}} Z_{\phi^{2}}^{-M} \Gamma^{(N, M)}
$$

our convention being that the unsubscripted quantity is the renormalized one. The parameters of the theory $t_{B}$ and $\lambda_{B}$ are multiplicatively renormalized, the relation between the bare and renormalized quantities being

$$
t=Z_{\phi^{2}}^{-1} t_{B} \quad \lambda=Z_{\lambda} \lambda_{B}
$$

$m_{B}^{2}$ must be additively renormalized, $m^{2}=m_{B}^{2}+\delta m_{B}^{2}$.

The renormalization factors are determined by the following generic normalization conditions

$$
\begin{gathered}
\left.\Gamma^{(2)}\right|_{\frac{p^{2}}{\kappa^{2}}=\alpha_{1}, \tau=\alpha_{2}}=\left(\alpha_{1}+\alpha_{2}\right) \kappa^{2} \\
\left.\frac{\partial \Gamma^{(2)}}{\partial p^{2}}\right|_{\frac{p^{2}}{\kappa^{2}}=\alpha_{1}, \tau=\alpha_{2}}=1 \\
\left.\Gamma^{(2,1)}\right|_{S P=\alpha_{1}, \tau=\alpha_{2}}=1 \\
\left.\Gamma^{(4)}\right|_{S P=\alpha_{1}, \tau=\alpha_{2}}=\lambda
\end{gathered}
$$

where $\tau=\frac{t}{\kappa^{2}}$, and $S P$ denotes $p_{i} \cdot p_{j}=\frac{\kappa^{2}}{4}\left(4 \delta_{i j}-1\right), p_{i}$ being the momentum entering through the $j$ th external leg, and $\kappa$ is an arbitrary renormalization scale. For the moment we will not specify the values of the various parameters involved in the normalization conditions. The alert reader might wonder why there is no condition corresponding to a renormalization of $g_{B}$. The answer is that in this paper we take it to be a RG invariant (i.e. a non-linear scaling field in the sense of Wegner [30]), though this in no way restricts the generality of our approach. We define the correlation length via the relation

$$
\xi^{2}=\frac{\int d^{d} x x^{2} G^{(2)}(x, 0)}{2 d \int d^{d} x G^{(2)}(x, 0)}
$$

where $G^{(2)}(x, 0)=\left.\frac{\delta^{2} W}{\delta J(x) \delta J(0)}\right|_{J=0}$. The conditions (2.7) and (2.8), together with $\alpha_{1}=0$, imply that $\xi$ defined by $(2.11)$ is $t^{-\frac{1}{2}}$ at the normalization point.

The wavefunction renormalization constant $Z_{\phi}$ is obtained from (2.8) by observing that since $Z_{\phi}$ is independent of $p$, and $\Gamma_{B}^{(2)}=Z_{\phi}^{-1} \Gamma^{(2)}$, then

$$
\left.\partial_{p^{2}} \Gamma_{B}^{(2)}\right|_{\frac{p^{2}}{\kappa^{2}}=\alpha_{1}, \tau=\alpha_{2}}=Z_{\phi}^{-1}
$$


Similarly (2.9) and (2.5) imply that

$$
Z_{\phi^{2}}^{-1}=\left.Z_{\phi} \Gamma_{B}^{(2,1)}\right|_{S P=\alpha_{1}, \tau=\alpha_{2}}
$$

whilst (2.10) and (2.5) imply that

$$
Z_{\lambda}=\left.Z_{\phi}^{2} \frac{\Gamma_{B}^{(4)}}{\lambda_{B}}\right|_{S P=\alpha_{1}, \tau=\alpha_{2}}
$$

The RG equation can be viewed as a simple consequence of the fact that the bare theory is independent of the arbitrary renormalization scale $\kappa$ at which we choose to define our parameters. Thus

$$
\kappa \frac{d}{d \kappa} \Gamma_{B}^{(N)}=0
$$

Using the relation between the bare and renormalized vertex functions (2.15) becomes

$$
\kappa \frac{d \Gamma^{(N)}}{d \kappa}-\frac{N}{2} \gamma_{\phi} \Gamma^{(N)}=0
$$

where

$$
\gamma_{\phi}=\frac{1}{Z_{\phi}} \kappa \frac{d Z_{\phi}}{d \kappa}
$$

Equation (2.16) has the formal solution

$$
\Gamma^{(N)}(\kappa)=e^{-\frac{N}{2} \int_{\kappa_{0}}^{\kappa} \gamma_{\phi}(x) \frac{d x}{x}} \Gamma^{(N)}\left(\kappa_{0}\right)
$$

We can further expand (2.16) by noting that there is implicit dependence on $\kappa$ through the renormalized couplings, and possible explicit dependence also. The differential equation then becomes

$$
\left(\kappa \frac{\partial}{\partial \kappa}+\beta \frac{\partial}{\partial \lambda}+\gamma_{\phi^{2}} t \frac{\partial}{\partial t}-\frac{1}{2} \gamma_{\phi}\left[N+\bar{\phi} \frac{\partial}{\partial \bar{\phi}}\right]\right) \Gamma^{(N)}=0
$$

with

$$
\gamma_{\phi^{2}}=\frac{1}{Z_{\phi^{2}}^{-1}} \kappa \frac{d Z_{\phi^{2}}^{-1}}{d \kappa}
$$

and

$$
\beta(\lambda)=\kappa \frac{d \lambda}{d \kappa}
$$

where

$$
\frac{\beta(\lambda)}{\lambda}=\gamma_{\lambda}=\frac{1}{Z_{\lambda}} \kappa \frac{d Z_{\lambda}}{d \kappa}
$$

$\lambda$ being the dimensionful coupling constant. The functions $\gamma_{\phi}, \gamma_{\phi^{2}}$ and $\gamma_{\lambda}$ are the Wilson functions. Note that a term $\frac{\partial}{\partial g}$ cannot appear due to the assumed non-renormalization of $g$. If we had chosen to parametrize the anisotropy by a coupling other than a non-linear 
scaling field, however, (2.19) would then be augmented by a term $\gamma_{g} g \frac{\partial}{\partial g}$. Equation (2.19) in conjunction with the dimensional analysis equation

$$
\rho \frac{d \Gamma^{(N)}}{d \rho}=N\left(\frac{d}{2}-1\right) \Gamma^{(N)}
$$

can be used to relate the correlation functions at two different scales.

\section{$\S$ 3. RENORMALIZATION GROUP AND THE "ENVIRONMENT"}

\section{$\S \S 3.1$ Comparison of Field Theoretic and "Wilsonian" RG's}

Before proceeding further let us consider the meaning of the RG equation (2.19). The RG, in the field theoretic context [31], expresses the invariance of physical quantities under a change of parameter. More generally it can be viewed as a reparametrization invariance of a differential equation and its boundary conditions [32]. It is an exact symmetry and therefore is a true invariance group of field theory. It is not unlike other reparametrization invariances. Viewed passively it expresses a triviality. Its power is that it can be used in conjunction with (2.23) to express a relationship between a parametrized system at two different scales. In contrast the RG transformation developed by Wilson and Kadanoff [33] is a mapping between probability distributions [34], realized as a mapping between renormalized Hamiltonians, and implemented by a "coarse graining" procedure such as momentum shell integration [35]. This Wilsonian RG is in fact a semi-group and only asymptotically represents an invariance of the system. That the two different approaches have led to essentially the same conclusions is a highly non-trivial result. The advantage of the Wilsonian methodology is that it is conceptually very clear, whilst the advantage of the field theoretic approach is that it is much easier to implement systematically, and perturbatively. The general motivation for using an RG approach is as a tool in understanding how systems with many degrees of freedom behave differently at different scales, one of the most fundamental facts about our universe being that it is full of "scales".

In the Wilson approach it is well known that there are many different types of RG, e.g. momentum shell integration, block spinning, majority rule etc. (see [36] for a recent review of the Wilsonian approach.) They are all realizable on some sufficiently large space of probability distributions, and correspond to maps from measures to measures. Usually, however, the Wilsonian RG is taken to be a map from Hamiltonians to Hamiltonians, and realizable as a flow on a sufficiently large parameter space $\mathcal{M}$. The general intuitive idea behind these transformations is that they are mappings (almost invariably approximate) between different effective degrees of freedom, represented by an effective Hamiltonian associated with different scales. If, for a particular system, each different scheme could be implemented, then universal quantities ought to be independent of the particular scheme used. An example of such a quantity would be a critical exponent, which is associated physically with a system that is almost scale invariant and is determined by a point in $\mathcal{M}$ which is associated with the scale invariant system (usually scale invariance also implies conformal invariance [37]). If the action of the RG operator is to be a "good" representation of the action of the dilatation operator then the fixed points of the RG transformation should be coincident in $\mathcal{M}$ with the points of scale invariance. That is not to say that they must have the same coordinates, but that the intrinsic geometry of the RG flows and the dilatation flows should be the same. For instance, if there were five points of scale invariance in $\mathcal{M}$, whereas the $\mathrm{RG}$ used only had three fixed points, then one could not think of the latter as being a very successful representation of scale changes. Additionally, 
the properties of the fixed points should be independent of the "coarse graining" procedure one uses, i.e. of the particular choice of RG.

Many systems, as a function of the parameters of the Hamiltonian describing the system, can exhibit scale invariance at more than one point of $\mathcal{M}$. Generally one then expects to see a crossover between different asymptotic regimes of the theory as governed by the various fixed points, hence, one is generically looking at the interpolation between two or more scale invariant field theories. If one considered a "coarse graining" procedure, such as momentum shell integration, for a system which possesses another length scale $g^{-1}$, other than the correlation length $\xi$, it is clear that the qualitative nature of the iterations of the RG should change as one considers momentum shells with $k \ll g$ and $k \gg g$. If one starts iterating at a scale $\Lambda \gg g$, then as the iteration proceeds into the IR, the RG flow will pass close to the $g=0$ fixed point (it will only actually hit it for $g=0$ ) before proceeding on to the $g=\infty$ fixed point. What is manifest is that a "physically sensible coarse graining" procedure will show up the qualitative change in the effective degrees of freedom as one considers different regimes of the iteration. The action of the "physically sensible coarse graining" follows in a reasonably faithful fashion the action of scale changes, hence one more generally may reasonably expect that there exists an RG that can successfully describe the change in behaviour of systems as one changes scale, mass scale, temperature scale, momentum scale etc. That the Wilson RG has been immensely successful is beyond question. Our purpose in the following is to construct such a field theoretic RG.

\section{$\S \S$ 3.2 Massive Versus Massless Renormalization}

Let us now turn back to the question of the field theoretic RG. If one considered a massive $\lambda \phi^{4}$ theory in three dimensions, one sets up the field theoretic RG formally in the same manner as shown in section 2. If one wishes to investigate the theory under changes in scale, and capture the points of scale invariance in $\mathcal{M}$, then one should look for fixed points of a suitable RG. There immediately arises the important question of what set of parameters will one's RG transformation depend on. This was not such an issue in the Wilsonian approach. There, in principle the RG transformation naturally depends on all the parameters of the effective Hamiltonian, in principle an infinite number, though usually it is sufficient to only consider it as a function of the relevant and marginal couplings, and these are usually finite in number.

In perturbative renormalization in field theory the story is somewhat different. In this setting there is a division of the role played by the Wilsonian RG transformation into two parts, represented by equations (2.19) and (2.23). If one accepts the philosophy that renormalization is solely a way of accounting for UV divergences, then one has a great deal of freedom as to what parameters one's counterterms should depend on, as the counterterms are effectively released from any dependence on IR scales. The idea then is to find the simplest form of (2.19) by making use of this freedom. The ultimate version of this type of approach is minimal subtraction, where one chooses as counterterms only those parts of diagrams that survive in the extreme UV limit. For the Hamiltonian (2.1), for instance, one knows that in the extreme UV counterterms that are mass independent are as good as mass dependent counterterms, if one's purpose is solely to perturbatively take the UV cutoff $\Lambda$ to infinity. This is because mass is an irrelevant coupling in the UV.

In our three dimensional example, only two fixed point are perturbatively accessible using mass independent schemes, the Gaussian and Wilson-Fisher fixed points with zero mass. If one uses mass dependent schemes, another fixed point becomes accessible - the infinite mass (temperature) Gaussian fixed point [38]. In fact these statements go beyond 
perturbation theory - if mass independent renormalization is used then the infinite mass Gaussian fixed point cannot be seen from the RG flow of the Wilson functions, if mass dependent renormalization is used then it can. In other words, in the mass independent renormalization the infinite mass Gaussian fixed point is not captured by the differential generator of (2.19), but relegated to a perturbative analysis of the vertex functions. Of course one can do such straight perturbation theory near the infinite mass limit, and in that sense there was no necessity to implement the RG. The reason one can get away with it, in the case of a mass operator, is that the critical exponents associated with the infinite mass Gaussian fixed point are mean field exponents, and therefore there was nothing extra to exponentiate, however, one is not always so lucky. Typically, if one tries to track the theory back into the IR having used a minimal subtraction scheme, in the presence of an additional mass scale, $g$, perturbation theory will break down, and some other procedure will be necessary. It is only the $g$ dependent $\mathrm{RG}$ which is capable of giving globally valid perturbative information.

More generally, if one has a field theory parametrized by a set of parameters, $P \equiv\left\{g^{i}\right\}$, corresponding to a point in $\mathcal{M}$, it might occur that different subsets of the parameters, relevant for describing the theory at different scales, are taken into one another by the RG flow on $\mathcal{M}$. If one's renormalization depends only on a subset of the parameters, one is restricting one's flow to take place only in a subspace $\mathcal{T}$ of $\mathcal{M}$. The resultant $\mathrm{RG}, R G_{\mathcal{T}}$, depends only on a subset $K$ of the parameters, and the RG flows take place only on $\mathcal{T}$. If any of the $P-K$ parameters are relevant in the RG sense, then the true RG flows of the theory, $R G_{\mathcal{M}}$, thought of as true scale changes, will wish to flow off $\mathcal{T}$ into $\mathcal{M}$. However, the use of $R G_{\mathcal{T}}$ does not allow for such flows. Such a state of affairs would be shown up by the perturbative unreliability of the results based on $R G_{\mathcal{T}}$. If none of the parameters $K$ are relevant then there should be no problem. However, one can only say what parameters are relevant when one knows the full fixed point structure of the theory! In principle it is obviously better to work with $R G_{\mathcal{M}}$. If a certain parameter was important then one has

made sure that its effects are treated properly, and if it wasn't then that will come out of the analysis. There can be no danger, except for extra work, from keeping a parameter in, but there can be severe problems if it is left out.

The message then is that in the field theoretic context one's choice of renormalization can be quite crucial, some points of scale invariance in $\mathcal{M}$ being inaccessible with respect to certain RG's. One might be rather worried by this, given that physics should be renormalization scheme independent. One must be careful to make a distinction between the points in $\mathcal{M}$, where the system under consideration is scale invariant, and those points which are fixed points of a particular RG. They are not necessarily the same. If one has chosen an RG which is a good representation of scale changes then they will be. We are not saying that there are conformally invariant systems that can only be accessed utilising certain RG's. What we are saying is that there exist RG's which are sufficient alone to describe the system. On the other hand, there are others that must be supplemented by extra non-perturbative information. For our massive field theory, the Wilson functions derived using mass dependent renormalization are sufficient on their own to access the mean field fixed point, whereas for mass independent schemes one has to supplement the Wilson functions with extra information from some other source, e.g. summing up possibly infinite sets of Feynman diagrams, in order to access the fixed point.

\section{$\S \S$ 3.3 The RG Should be Environment Dependent}

We can now perhaps take a somewhat broader view. In the above we discussed the fact that there were inequivalent field theoretic RG's, in the sense that the sets of fixed points of these RG's were not the same. Intuitively why is this, and why, in particular, does it 
happen in the field theoretic RG? Consider a physical system as we observe it at different scales. If we start at a very small scale $\Lambda^{-1}$, characterizing the system by a set of degrees of freedom (bare parameters), then try to describe the effective physics at some much larger scale $\kappa^{-1}$, it is almost invariably true, in a system with many degrees of freedom, that the physics at the scale $\kappa$ is very complicated in terms of the physics at the scale $\Lambda$. More often than not a better prescription is in terms of effective degrees of freedom, more appropriate for scales $\sim \kappa$. This is the whole raison d'etre behind effective field theory. Of course, experimentally one will find that the effective degrees of freedom are very different at different scales. In the above, the effective degrees of freedom at scales $\kappa \gg m$ are essentially massless with power law correlations, whereas for $\kappa \ll m$ they are essentially decoupled. This is a statement about what one would see experimentally, different sorts of correlations in different asymptotic regimes. Clearly one would like one's calculational schemes to capture this experimental fact - different effective degrees of freedom at different scales. Though the Wilson RG was originally developed to tackle systems with just the opposite problem - effective degrees of freedom that are the same at all scales (!) - a "good coarse graining" procedure will pick up the change in effective degrees of freedom. Similarly, we will show that a "good" field theoretic RG can follow the changing effective degrees of freedom.

We mentioned above that the field theoretic formalism is calculationally much simpler, but have gone into some detail about potential pitfalls in using it. In the rest of the paper we will see these pitfalls discussed in a much less heuristic fashion than here and also see how they can be avoided. The goal then is clear: to develop field theoretic RGs who's fixed points encompass all the points of scale invariance for a particular system. The above discussion of massless versus massive field theoretic RGs is just an illustrative example, our main concern in this paper will be dimensional crossover, however, similar considerations hold much more generally as we will now discuss.

What one considers to be fluctuations in a system are not unique. Fluctuations are usually defined with respect to some "background", or in the terminology we will adopt here, some "environment". The environment can have many different properties. One of the first things one might ask is what space (or spacetime) does the environment live in. Here we want to be more general and consider the space itself as part of the environment. Thus if one works in infinite flat space in three dimensions, $R^{3}$, or in a "box" of size $L$ $\left(L^{3}\right)$, then the properties of the space should be counted as part of the environment. The reason is obvious. Fluctuations of a field are qualitatively different in $R^{3}$ and $L^{3}$. If one's only concern is UV divergences then certainly fluctuations with momenta $k \gg L^{-1}$ are qualitatively the same on either $R^{3}$ or $L^{3}$, however, for momenta $k \sim L^{-1}$ the qualitative difference is great, and for $k \ll L^{-1}$ it is absolutely profound as such modes don't even exist on $L^{3}$ ! Clearly then the effects of the environment are very scale dependent. If one renormalizes one's field theory on $L^{3}$ using only minimal subtraction say, then, the information one gets out from the consequent RG will be information appropriate to the field theory on $R^{3}$, not $L^{3}$. Hence we will say that minimal subtraction is not environmentally friendly on $L^{3}$, the environmental variable here being $L$. One could have, of course, an anisotropic box with different size sides, then one would have three environmental variables. A proper description of the fluctuations from a RG standpoint would demand that the RG depended on these three variables. We could consider a space with curvature (constant or not), this would add additional environment dependence. This would be of some relevance in the early universe, where the cosmological constant can also be regarded as an environmental variable. The IR behaviour of quantum fields in inflation would only be accessible using RG methods using an RG that depended on the cosmological constant. 
So, one of the most important types of environmental variable comes from properties of the space one works in. A second type comes from what sort of background fields are present. A gravitational background field is obviously related to the above question of what space one is working in. One could also consider the fluctuations of a charged field say, coupled to a background electric or magnetic field. The spectrum of fluctuations in a uniform magnetic field $B$, for instance, is completely different to that for $B=0$. The $B$ field sets a length scale, and fluctuations with wavelengths very large or small relative to it are very different. In fact one can think of the $B$ field as making the fluctuations live in a "box" [39]. The particular background could of course be much more complicated than a simple constant field. One example would be the Abrikosov flux lattice, a background of monopoles another, the latter possibly being of great relevance for QCD. In other words when one is looking at QCD, then the way quarks and gluons behave in a background of monopoles will be very different to their behaviour in no background. The standard renormalization of QCD, using minimal subtraction, is really only appropriate for the situation where there is no background, or one is looking at length scales much shorter than the characteristic scale of the background, i.e. at high energy - asymptotic freedom - where quarks and gluons are a good representation of the effective degrees of freedom.

In the above we have given some examples of environment and maintained that the environment on certain length scales will greatly influence the nature of the effective degrees of freedom which are a description of the system. Quite often the environmental variables can be thought of as setting a fixed length scale, or set of scales, which lead to fluctuations in the presence of this environment that are qualitatively different at different scales. In this paper we will be exhibiting a RG methodology which can describe such changes. However, the above example of QCD illustrates another important point, and that is that in many cases in practise, one might not be able to make a very clean distinction between fluctuations and environment as they interact dynamically. Not only does the environment influence the fluctuations but also the fluctuations feed back into the environment. Quite often this feedback is negligible, however, when it is not then there needs to be a feedback mechanism between the RG as a function of the environment and the environment as a function of the fluctuations. A simple example of such a state of affairs is $\lambda \phi^{4}$ below the critical point. In such a situation the fluctuations influence the background field and one can investigate this explicitly via the equation of state. It is the equation of state that tells one how the fluctuations are reacting back on the environment, the environment in this case being the background magnetization. For more complicated systems there will be more "equations of state", these must be solved in conjunction with the environment dependent RG equations to get a closed system. Obviously more often than not this will not be easy.

We hope the idea of environmentally friendly renormalization is understandable and hope the reader by the end of the paper will see the necessity of it.

\section{§ 4. FORMAL PERTURBATION THEORY}

\section{$\S \S 4.1$ Determination of Renormalization Constants}

In this section we will present formal diagramatic series for the correlation functions and the renormalization constants. We will assume the generic normalization conditions of section 2, but for now will not specify the precise parameter/environment dependence of them. The following considerations will be largely independent of such things and therefore applicable to a large class of crossover problems. The expressions apply to crossovers where 
the number of symmetry components of the order parameter doesn't change, however, the changes needed to encapsulate this larger class of crossovers are small [18].

The effective action (free energy) (2.3) is given by

$$
\Gamma=S+\Sigma
$$

where $S$ is the classical action (mean field Hamiltonian) and $\Sigma$ represents the contribution due to fluctuations. The diagramatic series for the effective action can be constructed in a loop expansion which gives a series ordered by the number of loops. For the $O(N)$ model under consideration the quadratic part of the Hamiltonian is defined by an operator

$$
A^{a b}=A_{1} P_{1}^{a b}+A_{2} P_{2}^{a b}
$$

where the two projection operators $P_{1}$ and $P_{2}$ are

$$
P_{1}^{a b}=\frac{\phi_{B}^{a} \phi_{B}^{b}}{\phi_{B}^{2}} \quad \text { and } \quad P_{2}^{a b}=\delta^{a b}-\frac{\phi_{B}^{a} \phi_{B}^{b}}{\phi_{B}^{2}}
$$

We denote the inverse of $A^{a b}$

$$
G^{a b}=G_{1} P_{1}^{a b}+G_{2} P_{2}^{a b}
$$

For the case of dimensional crossover which we will consider in section 11

$$
A^{a b}=\left(\boldsymbol{\square}+m_{B}^{2}+t_{B}+\frac{\lambda_{B}}{2} \phi_{B}^{2}\right) P_{1}^{a b}+\left(\boldsymbol{\square}+m_{B}^{2}+t_{B}+\frac{\lambda_{B}}{6} \phi_{B}^{2}\right) P_{2}^{a b}
$$

which gives

$$
G_{1}=\frac{1}{\square+m_{B}^{2}+t_{B}+\frac{\lambda_{B}}{2} \phi_{B}^{2}} \quad \text { and } \quad G_{2}=\frac{1}{\square+m_{B}^{2}+t_{B}+\frac{\lambda_{B}}{6} \phi_{B}^{2}}
$$

where $\square$ is the Laplace operator in the geometry of interest. The effective action to two loops is given by

$$
\begin{aligned}
\Gamma= & \int d x \frac{1}{2}\left[\phi_{B}\left(\square+m_{B}^{2}+t_{B}\right) \phi_{B}(x)+\frac{\lambda_{B}}{4 !} \phi_{B}^{4}(x)\right]+\frac{1}{2} \operatorname{Tr} \ln \left[A_{1}\right]+\frac{1}{2}(N-1) \operatorname{Tr} \ln \left[A_{2}\right] \\
& +\frac{\lambda_{B}}{4 !} \int d x\left[3 G_{1}^{2}(x, x)+2(N-1) G_{1}(x, x) G_{2}(x, x)+\left(N^{2}-1\right) G_{2}^{2}(x, x)\right] \\
& -\frac{\lambda_{B}^{2}}{36} \int d x \int d y \phi_{B}(x)\left[3 G_{1}^{3}(x, y)+(N-1) G_{1}(x, y) G_{2}^{2}(x, y)\right] \phi_{B}(y)
\end{aligned}
$$

The contributions to the respective $\Gamma^{(N)}$ can be obtained by differentiating with respect to $\phi_{B}(x)$ then setting $\phi_{B}=\bar{\phi}_{B}$, where $\bar{\phi}_{B}$ is the solution of $\Gamma_{B}^{(1)}\left(\bar{\phi}_{B}\right)=H_{B}$. The $\Gamma^{(N, M)}$ can further be generated by differentiation with respect to $t_{B}(x)$. Specializing now to constant magnetic field $H_{B}$; the magnetization $\bar{\phi}_{B}$ will also be constant for a homogenous 
and isotropic environment, which we will now assume for simplicity. The effective potential (free energy density) to two loops is then given by

$$
\begin{aligned}
\mathcal{F}= & \frac{1}{2}\left(m_{B}^{2}+t_{B}\right) \bar{\phi}_{B}^{2}+\frac{\lambda_{B}}{4 !} \bar{\phi}_{B}^{4}+\frac{1}{2} \operatorname{tr} \ln \left[A_{1}\right]+\frac{1}{2}(N-1) \operatorname{tr} \ln \left[A_{2}\right] \\
+ & \frac{\lambda_{B}}{4 !} \frac{1}{V} \int d x\left[3 G_{1}^{2}(x, x)+2(N-1) G_{1}(x, x) G_{2}(x, x)+\left(N^{2}-1\right) G_{2}^{2}(x, x)\right] \\
& -\frac{\lambda_{B}^{2}}{36} \frac{1}{V} \int d x \int d y \bar{\phi}_{B}\left[3 G_{1}^{3}(x, y)+(N-1) G_{1}(x, y) G_{2}^{2}(x, y)\right] \bar{\phi}_{B}
\end{aligned}
$$

where $V$ is the volume of the system and $t r=\frac{T r}{V}$.

We will now restrict our considerations to situations where $\bar{\phi}_{B}=0$, which corresponds to being above the critical temperature with $H_{B}=0$, once again this in no way restricts the generality of our methodology [29]. In this case we also have $G_{1}=G_{2}=G$, and the vertex functions are proportional to $O(N)$ symmetric tensors. We will concentrate on the coefficient of the relevant tensor eliminating the tensorial structure altogether for simplicity. Thus adopting a suggestive diagramatic notation (see appendix A for an explanation and for the actual expressions in the dimensional crossover problem) and working with dimensionless diagrams by pulling out powers of an arbitrary scale $\kappa$, one finds the one loop contributions are given by

$$
\begin{gathered}
\mathcal{F}_{1}=\frac{N}{2} \kappa^{d} \bigcirc \\
\Gamma_{1}^{(2)}=\frac{(N+2)}{6} \lambda_{B} \kappa^{d-2} \bigcirc \\
\Gamma_{1}^{(4)}=-\frac{3}{2}\left(\frac{N+8}{9}\right) \lambda_{B}^{2} \kappa^{4-d} \bigcirc
\end{gathered}
$$

The two loop contribution is

$$
\begin{gathered}
\mathcal{F}_{2}=\frac{\lambda_{B}}{4 !} N(N+2) \kappa^{2 d-4} \bigcirc^{2} \\
\Gamma_{2}^{(2)}=-\frac{\lambda_{B}^{2}}{36} \kappa^{2 d-6}(N+2)((N+2) \bigcirc \bigcirc+2 \ominus)
\end{gathered}
$$

and

$$
\left.\Gamma_{2}^{(4)}=\frac{\lambda_{B}^{3}}{36} \kappa^{2(d-4)}\left(\left(N^{2}+6 N+20\right) \bigcirc^{2}+2(N+2)(N+8) \bigcirc \bigcirc+4(5 N+22) \downarrow\right)\right)
$$

Putting the zero, one and two loop contributions together, we are in a position to construct the transformation to renormalized parameters, however, our main concern here is to generate the renormalization constants so that the Wilson functions may be calculated. An important observation is that for an amputated diagram it is only the number of insertions that is relevant not the operator inserted. We have not explicitly exhibited the different momentum combinations that contribute to $\Gamma^{(4)}$ as separate diagrams, the sum over the different combinations of momenta being implicit. The diagrams as written are therefore to be understood as averaged over the Mandelstam variables, which at the symmetric point all become equal. Thus the diagramatic series formally take on their 
symmetric point form. The reader should however be careful to remember this point when implementing expressions for the formal series at a non-symmetric point.

Adding the different loop contributions together gives

$$
\begin{gathered}
\mathcal{F}=\frac{N}{2} \kappa^{d}\left(\bigcirc+\frac{\lambda_{B}}{12} \kappa^{d-4}(N+2) \bigcirc^{2}\right) \\
\Gamma_{B}^{(2)}=p^{2}+m_{B}^{2}+t_{B}+\frac{\lambda_{B}}{6}(N+2) \kappa^{d-2}\left(\bigcirc-\frac{\lambda_{B} \kappa^{d-4}}{6}((N+2) \bigcirc \bigcirc+2 \bigcirc)\right) \\
\Gamma_{B}^{(2,1)}=1-\frac{\lambda_{B} \kappa^{d-4}}{6}(N+2)\left(\bigcirc-\frac{\lambda_{B}}{3} \kappa^{d-4}(N+2) \bigcirc: \bigcirc\right) \\
\left.+\frac{\lambda_{B}^{2}}{36}(N+2) \kappa^{2(d-4)}\left((N+2) \bigcirc^{2}+6 \sqrt{ }\right)\right) \\
\frac{\partial \Gamma_{B}^{(2)}}{\partial p^{2}}=1-\frac{\lambda_{B}^{2}}{18} \kappa^{2(d-4)}(N+2) \phi
\end{gathered}
$$

and

$$
\begin{array}{r}
\Gamma_{B}^{(4)}=\lambda_{B}-\frac{\lambda_{B}^{2}}{6}(N+8) \kappa^{d-4}\left(\bigcirc-\frac{\lambda_{B}}{3} \kappa^{d-4}(N+2) \bigcirc: \bigcirc\right) \\
\left.+\frac{\lambda_{B}^{3}}{36} \kappa^{2(d-4)}\left(\left(N^{2}+6 N+20\right) \bigcirc^{2}+4(5 N+22) \downarrow\right)\right)
\end{array}
$$

where we have regrouped the two loop tadpole terms with the corresponding one loop terms.

The Wilson functions are derived from the renormalization constants $Z_{\phi}, Z_{\phi^{2}}$ and $Z_{\lambda}$, however, as mentioned in section 2 an additive mass renormalization must also be performed, which corresponds in critical phenomena language to a shift in "reference" temperature from the mean field critical temperature to some other, usually the "true" critical temperature, if such exists. We begin with

$$
\Gamma_{B}^{(2)}\left(p, m_{B}^{2}+t_{B}\right)=p^{2}+m_{B}^{2}+t_{B}+\Sigma_{B}^{(2)}\left(p, m_{B}^{2}+t_{B}\right)
$$

where $\Sigma_{B}^{(2)}$ is the contribution from fluctuations. There are two undetermined parameters in $\Gamma^{(2)}, m_{B}^{2}$ and the scale of $t_{B}$. We find these by imposing two normalization conditions. The first to be determined is the additive renormalization given by $m^{2}=m_{B}^{2}+\delta m_{B}^{2}$, where $m^{2}$ is the value of $\Gamma^{(2)}$ at $t=0$ and $p=0$. We will in fact take $m^{2}=0$. Therefore we determine $\delta m_{B}^{2}$ by requiring that

$$
\delta m_{B}^{2}=\Sigma_{B}^{(2)}\left(0, \delta m_{B}^{2}\right)
$$

An important point to make here is that both $m_{B}^{2}$ and $\delta m_{B}^{2}$ are $p$ and $t$ independent, a fact that we will use to our advantage later. Condition 4.18, perturbatively for $d<4$, is very difficult to treat to the presence of IR divergences. The requirement that $\Gamma^{(2)}$ be zero 
when $t=0$ and $p=0$ can only be used, of course, if the system exhibits critical behaviour in the specific environmental setting under study. We then have, as usual, that $t_{B}$ is a linear measure of the deviation from the critical temperature.

Recall that

$$
\Gamma_{B}^{(2)}\left(p, m_{B}^{2}+t_{B}\right)=\sum_{M} \frac{1}{M !} \Gamma^{(2, M)}\left(p, m_{B}^{2}\right) t_{B}^{M}
$$

This implies that

$$
0=\Gamma_{B}^{(2,0)}\left(0, m_{B}^{2}\right)
$$

which is our specification of $\delta m_{B}^{2}$, however, we can equally well write (4.19) in the form

$$
\Gamma_{B}^{(2)}\left(p, m_{B}^{2}+t_{B}\right)=\sum_{M} \frac{1}{M !} \Gamma_{B}^{(2, M)}\left(p, m_{B}^{2}+\Lambda^{2}\right)\left(t_{B}-\Lambda^{2}\right)^{M}
$$

where we have just chosen to Taylor expand around a different point, $\Lambda$ here being some fixed scale. This latter equation implies that at $t_{B}=\Lambda^{2}$

$$
\Gamma_{B}^{(2)}\left(0, m_{B}^{2}+\Lambda^{2}\right)=\Gamma_{B}^{(2,0)}\left(0, m_{B}^{2}+\Lambda^{2}\right)
$$

All this says is that we can choose any fiducial ( $R G$ invariant) point to measure deviations in temperature/mass with respect to. It just so happens that if there exists a critical point the critical temperature is in some sense a preferred point around which to measure deviations.

Another condition which could be used to determine $\delta m_{B}^{2}$ is

$$
\Gamma^{(2)}\left(0, t\left(\kappa_{c}\right)=\kappa_{c}^{2}\right)=\kappa_{c}^{2}
$$

which corresponds to $\alpha_{1}=0, \alpha_{2}=\frac{\kappa_{c}^{2}}{\kappa^{2}}$. Here the scale $\kappa_{c}$ is a fixed scale being the value of the renormalization scale which satisfies the equation $t\left(\kappa_{c}\right)=\kappa_{c}^{2}, t\left(\kappa_{c}\right)$ being the running temperature evaluated at the scale $\kappa_{c}$. Equation (4.20) gives $\delta m_{B}^{2}$ to be a solution of

$$
Z_{\phi}^{-1} \kappa_{c}^{2}=m^{2}+\delta m_{B}^{2}+Z_{\phi^{2}}^{-1} \kappa_{c}^{2}+\Sigma\left(0, m^{2}+\delta m_{B}^{2}+Z_{\phi^{2}}^{-1} \kappa_{c}^{2}\right)
$$

If there exists a true critical point, then as the critical temperature is approached, one finds that, as $\kappa_{c} \rightarrow 0$, the above condition becomes equivalent to (4.18).

The condition (4.18) is to be solved perturbatively to yield the "shift" from the mean field critical temperature to another critical temperature. The particular temperature one shifts to depends on the values of the environmental parameters, such as $g$, entering the normalization condition. This is natural because $T_{c}$ generically depends on environmental variables, therefore as the environment changes, the relevant $T_{c}$ to which it is appropriate to shift to changes accordingly. The shift, although intersting, is not something we will consider in this paper. The upshot of all the above is that $\bigcirc . \bigcirc$ in (4.14) and (4.16) cancel with the mass counterterm associated with the propagators in the one loop diagrams $\bigcirc$ and $\bigcirc$. In fact a similar cancellation will occur order by order in perturbation theory. 
Using the condition $\Gamma^{(2)}(p=0, t=0)=0$ gives

$$
\begin{aligned}
& \Gamma_{B}^{(2)}=p^{2}+t_{B} \\
& \quad-\frac{\lambda_{B}^{2}}{36}(N+2) \kappa^{2 d-6}\left\{(N+2)\left(\bigcirc \bigcirc-\left.\left.\bigcirc\right|_{0} \bigcirc\right|_{0}\right)+2\left(\ominus-\left.\ominus\right|_{0}\right)\right\}
\end{aligned}
$$

where the subscript 0 indicates $p=t_{B}=0$. However, as mentioned previously this has potential problems with IR divergences. An analogous expression which is free of this problem follows from (4.21).

$\Gamma_{B}^{(4)}$ and $\Gamma_{B}^{(2,1)}$ at the generic normalization points of section 2 are

$$
\begin{aligned}
\Gamma_{B}^{(4)}= & \lambda_{B}-\frac{(N+8)}{6} \lambda_{B}^{2} \kappa^{d-4} \bigcirc \\
& \left.+\frac{(5 N+22)}{9} \lambda_{B}^{3} \kappa^{2(d-4)} \downarrow\right)+\frac{\left(N^{2}+6 N+20\right)}{36} \lambda_{B}^{3} \kappa^{2(d-4)} \bigcirc^{2}
\end{aligned}
$$

and

$$
\left.\Gamma_{B}^{(2,1)}=1-\frac{(N+2)}{6} \lambda_{B} \kappa^{d-4} \bigcirc+\frac{(N+2)}{6} \lambda_{B}^{2} \kappa^{2(d-4)}(\mathcal{V})+\frac{(N+2)}{6} \bigcirc^{2}\right)
$$

Remember that the diagrams in these expressions are now to be evaluated at the appropriate normalization point, explicit expressions for the case of a layered geometry can be found in the appendices.

Differentiating (4.22) with respect to $p^{2}$, then setting the condition (2.8) yields in the two loop approximation

$$
Z_{\phi}=1+\frac{(N+2)}{18} \lambda_{B}^{2} \kappa^{2(d-4)} \phi+\ldots
$$

From (4.24) one finds

$$
\begin{aligned}
Z_{\phi}^{-1} Z_{\phi^{2}}^{-1}= & -\frac{(N+2)}{6} \lambda_{B} \kappa^{d-4} \bigcirc \\
& +\frac{(N+2)^{2}}{36} \lambda_{B}^{2} \kappa^{2(d-4)} \bigcirc^{2}+\frac{(N+2)}{6} \lambda_{B}^{2} \kappa^{2(d-4)}
\end{aligned}
$$

and hence

$$
\begin{aligned}
Z_{\phi^{2}}^{-1}=1- & \frac{(N+2)}{6} \lambda_{B} \kappa^{d-4} \bigcirc+\frac{(N+2)^{2}}{36} \lambda_{B}^{2} \kappa^{2(d-4)} \bigcirc^{2} \\
+ & \left.\frac{(N+2)}{6} \lambda_{B}^{2} \kappa^{2(d-4)}(v)+\frac{1}{3} \phi\right)
\end{aligned}
$$

The renormalized four point function, including wavefunction renormalization yields

$$
\begin{aligned}
\Gamma_{B}^{(4)} & =\lambda_{B}-\frac{(N+8)}{6} \lambda_{B}^{2} \kappa^{d-4} \bigcirc \\
& \left.+\frac{\lambda_{B}^{3}}{9} \kappa^{2(d-4)}((5 N+22) \mathcal{V})+\frac{\left(N^{2}+6 N+20\right)}{4} \bigcirc^{2}+(N+2) \phi\right)
\end{aligned}
$$


therefore

$$
\begin{aligned}
Z_{\lambda}= & -\frac{(N+8)}{6} \lambda_{B} \kappa^{d-4} \bigcirc \\
& \left.+\lambda_{B}^{2} \kappa^{2(d-4)}\left(\frac{(5 N+22)}{9} \downarrow\right)+\frac{\left(N^{2}+6 N+20\right)}{36} \bigcirc^{2}+\frac{(N+2)}{9} \phi\right)
\end{aligned}
$$

It is obviously trivial to obtain the renormalization constants in terms of the renormalized coupling. We leave them in terms of the bare coupling as this coupling that must be kept constant when evaluating the Wilson functions.

\section{$\S \S 4.2$ Diagramatic Expansion of the Wilson Functions}

Having derived the renormalization constants we are now in a position to calculate the Wilson functions. From the definition of $\gamma_{\phi^{2}}$ (2.20), using (4.26) one finds

$$
\begin{aligned}
\gamma_{\phi^{2}} & =-\frac{(N+2)}{6}\left(A_{(4-d)} \bigcirc\right) \kappa^{d-4} \lambda \\
& +\frac{(N+2)}{6}\left(A_{2(4-d)}\left(()-\frac{1}{2} \bigcirc^{2}\right)+\frac{1}{3} A_{2(4-d)} \phi\right) \kappa^{2(d-4)} \lambda^{2}
\end{aligned}
$$

where we have defined

$$
A_{\nu}=\kappa^{\nu} \kappa \frac{d}{d \kappa} \kappa^{-\nu}=\kappa \frac{d}{d \kappa}-\nu
$$

and we now work in terms of the renormalized coupling $\lambda$. Similarly, from the definition of $\gamma_{\phi}(2.17)$, and using (4.25) one gets

$$
\gamma_{\phi}=\frac{(N+2)}{18}\left(A_{2(4-d)} \phi\right) \kappa^{2(d-4)} \lambda^{2}
$$

and finally from (2.21), using (4.28) one obtains for $\gamma_{\lambda}$

$$
\begin{aligned}
\gamma_{\lambda}= & -\frac{(N+8)}{6}\left(A_{(4-d)} \bigcirc\right) \kappa^{(d-4)} \lambda \\
& \left.+\left(\frac{(5 N+22)}{9} A_{2(4-d)}(\mathbb{N})-\frac{1}{2} \bigcirc^{2}\right)+\frac{(N+2)}{9} A_{2(4-d)} \phi\right) \kappa^{2(d-4)} \lambda^{2}
\end{aligned}
$$

From the definition of $\gamma_{\lambda}$ and its relation to the $\beta$ function one obtains the $\beta$ function of the dimensionful coupling

$$
\begin{aligned}
\kappa \frac{d \lambda}{d \kappa}= & -\frac{(N+8)}{6}\left(A_{(4-d)} \bigcirc\right) \kappa^{(d-4)} \lambda^{2} \\
& \left.+\left(\frac{(5 N+22)}{9} A_{2(4-d)}(1)-\frac{1}{2} \bigcirc^{2}\right)+\frac{(N+2)}{9} A_{2(4-d)} \phi\right) \kappa^{2(d-4)} \lambda^{3}
\end{aligned}
$$

We now introduce the dimensionless coupling constant $\bar{\lambda}=\lambda \kappa^{d-4}$, the $\beta$ function of which is

$$
\begin{aligned}
\kappa \frac{d \bar{\lambda}}{d \kappa}= & -(4-d) \bar{\lambda}-\frac{(N+8)}{6}\left(A_{(4-d)} \bigcirc\right) \bar{\lambda}^{2} \\
& +\left(\frac{(5 N+22)}{9} A_{2(4-d)}\left(\mathcal{V}-\frac{1}{2} \bigcirc^{2}\right)+\frac{(N+2)}{9} A_{2(4-d)} \phi\right) \bar{\lambda}^{3}
\end{aligned}
$$


We stress that these expressions for the Wilson functions are totally finite for $d \leq 4$.

We now introduce another coupling [27] by performing a coordinate transformation on $\mathcal{M}$. The new coupling $h$, which we dub the floating coupling, is defined so as to make the coefficient of the quadratic term in $\beta(h)$ unity. From (4.33) this is achieved via the redefinition $h=-\frac{(N+8)}{6} A_{4-d} \bigcirc \bar{\lambda}$. Thus the $\beta$-function for this coupling is

$$
\beta(h)=-\varepsilon(\bigcirc) h+h^{2}+\frac{4\left((5 N+22) A_{2(4-d)}\left(\mathcal{V}-\frac{1}{2} \bigcirc^{2}\right)+(N+2) A_{2(4-d)} \phi\right)}{\left((N+8) A_{4-d} \bigcirc\right)^{2}} h^{3}
$$

where

$$
\varepsilon(\bigcirc)=4-d-\kappa \frac{d \ln A_{4-d} \bigcirc}{d \kappa}
$$

Note that in the limit $N \rightarrow \infty$ the $\beta$-function for this coupling becomes exact at the quadratic order. The condition $\beta(h)=0$ defines what we call the floating fixed point, the significance of which will be seen shortly. Note that in fixed dimension flat space for massless propagators, or where the mass is taken to be the scale of our normalization point, $A_{4-d} \bigcirc$ and $A_{2(4-d)}\left(\mathcal{V}-\frac{1}{2} \bigcirc^{2}\right)$ are just numbers. Finally we introduce for later convenience two combinations of diagrams, $f_{1}$ and $f_{2}$ defined by

$$
\begin{gathered}
f_{1}=-\frac{\left.A_{2(4-d)}(\downarrow)-\frac{1}{2} \bigcirc^{2}\right)}{\left(A_{4-d} \bigcirc\right)^{2}} \\
f_{2}=\frac{A_{2(4-d)} \phi}{\left(A_{4-d} \bigcirc\right)^{2}}
\end{gathered}
$$

\section{$\S$ 5. ENVIRONMENT DEPENDENT RENORMALIZATION}

\section{$\S \S 5.1$ "Running" Mass/Temperature or Momentum}

In sections 2 through 4 we have set up the machinery for investigating crossovers in a formal fashion and without specializing to a particular one. At this point we want to put some flesh on the bare bones of the formalism. Remember that our ultimate goal is to be able to describe systems that exhibit qualitatively different effective degrees of freedom at different scales. This necessarily implies that the effective degrees of freedom are sensitive to the environment. In section 3 we had a preliminary discussion of this in the context of comparing and contrasting the field theoretic RG with the Wilson RG. The previous sections were the embodiment of the former implemented perturbatively. So how does one see the concept of different RG's and enviroment dependence coming out here? Obviously it is the fluctuations of the system that are sensitive to the environment and it is these very fluctuations that we are cavalierly calling effective degrees of freedom. It should be clear then that it is the actual functional forms of the Feynman diagrams themselves that exhibit this dependence.

So how do different RG's appear? This depends crucially on one's normalization conditions. If, as discussed in section 2 , there exists an important mass scale $g$, then physics at scales $\kappa \ll g$ and $\kappa \gg g$ will be very different. We said earlier that if one's goal is 
to describe systems at different scales then the RG is in principle an ideal tool for the investigation. A good RG will be one who's fixed points represent all the points of scale invariance of the physical system in $\mathcal{M}$. If we chose renormalization conditions which were explicitly $g$ independent, i.e. we used the generic normalization conditions of section 2 with $g=0$, for example

$$
\left.\Gamma^{(2)}(p, t, \lambda, g=0, \kappa)\right|_{\frac{p^{2}}{\kappa^{2}}=\alpha_{1}, \tau=\alpha_{2}}=\left(\alpha_{1}+\alpha_{2}\right) \kappa^{2}
$$

and analogously for the other normalization conditions, then the Wilson functions would be found to be independent of $g$. This we emphasize is not just a perturbative failing. If the renormalization schemes used are $g$ independent then so are the resulting Wilson functions and that is that. If we use $g$ dependent renormalization conditions, however, then the resulting Wilson functions will be explicitly dependent on $\frac{g}{\kappa}$.

To be more concrete: consider $g$ to be a fixed "physical" scale (i.e. it doesn't get renormalized as mentioned in section 1 ). We will choose the normalization conditions

$$
\begin{gathered}
\Gamma^{(2)}\left(p=0, t=\kappa^{2}, \bar{\lambda}, g, \kappa\right)=\kappa^{2} \\
\left.\frac{\partial \Gamma^{(2)}}{\partial p^{2}}\left(p, t=\kappa^{2}, \bar{\lambda}, g, \kappa\right)\right|_{p=0}=1 \\
\Gamma^{(4)}\left(p=0, t=\kappa^{2}, \bar{\lambda}, g, \kappa\right)=\bar{\lambda} \kappa^{4-d} \\
\Gamma^{(2,1)}\left(p=0, t=\kappa^{2}, \bar{\lambda}, g, \kappa\right)=1
\end{gathered}
$$

Condition (5.2), together with the multiplicative renormalization of $t$, implies that $t$ is proportional to $T-T_{c}(g)$, i.e. that one is measuring temperature relative to the $g$ dependent critical point (we are assuming that the system can exhibit critical behaviour for any value of $g$ ). If an analogous condition with $g=0$ had been used, temperature would be consequently measured relative to $T_{c}(0)$, the critical temperature of the isotropic system. As these conditions are explicitly $g$ dependent, the RG equation (2.19) for an $N$-point vertex function takes the form

$$
\left(\kappa \frac{\partial}{\partial \kappa}+\beta\left(\bar{\lambda}, \frac{g}{\kappa}\right) \frac{\partial}{\partial \bar{\lambda}}+\gamma_{\phi^{2}}\left(\bar{\lambda}, \frac{g}{\kappa}\right) t \frac{\partial}{\partial t}-\frac{1}{2} \gamma_{\phi}\left(\bar{\lambda}, \frac{g}{\kappa}\right)\left(N+\bar{\phi} \frac{\partial}{\partial \bar{\phi}}\right)\right) \Gamma^{(N)}=0
$$

where now the Wilson functions $\beta, \gamma_{\phi}$ and $\gamma_{\phi^{2}}$ not only depend on $\bar{\lambda}$, the dimensionless renormalized $\phi^{4}$ coupling, in the standard fashion, but also on $\frac{g}{\kappa}$. Notice that 5.2-5.5 imply that the parameter which is generating the flow here is the renormalized mass/temperature parameter. We could equally well of course have used external momentum to generate the flow.

The RG equation can be solved in the standard manner using the method of characteristics to yield

$$
\begin{gathered}
\Gamma^{(N)}\left(p_{i}, t, \bar{\phi}, \bar{\lambda}, g, \kappa\right)=(\kappa \rho)^{d-N \frac{(d-2)}{2}} \exp \left(-\frac{N}{2} \int_{1}^{\rho} \gamma_{\phi}\left(\bar{\lambda}(x), \frac{g}{\kappa x}\right) \frac{d x}{x}\right) \\
\Gamma^{(N)}\left(\frac{p_{i}}{\rho \kappa}, \frac{t(\rho)}{\rho^{2} \kappa^{2}}, \frac{\bar{\lambda}(\rho) \bar{\phi}^{2}(\rho)}{2(\rho \kappa)^{d-2}}, \bar{\lambda}(\rho), \frac{g}{\rho \kappa}, 1\right)
\end{gathered}
$$


where $t(\rho), \bar{\lambda}(\rho)$, and $\bar{\phi}(\rho)$; the running temperature, coupling and magnetization respectively, satisfy

$$
\begin{gathered}
\rho \frac{d \bar{\lambda}(\rho)}{d \rho}=\beta\left(\bar{\lambda}(\rho), \frac{g}{\kappa \rho}\right) \\
\rho \frac{d t(\rho)}{d \rho}=\gamma_{\phi^{2}}\left(\bar{\lambda}(\rho), \frac{g}{\kappa \rho}\right) t(\rho) \\
\rho \frac{d \bar{\phi}(\rho)}{d \rho}=-\frac{1}{2} \gamma_{\phi}\left(\bar{\lambda}(\rho), \frac{g}{\kappa \rho}\right) \bar{\phi}(\rho)
\end{gathered}
$$

With a $g$ dependent renormalization condition such as (5.4) the $\beta$ function for the dimensionless coupling will generically have the form

$$
\rho \frac{d \bar{\lambda}(\rho)}{d \rho}=-(4-d) \bar{\lambda}(\rho)+a_{2}\left(\frac{g}{\kappa \rho}\right) \bar{\lambda}^{2}(\rho)+\sum_{n=3}^{\infty} a_{n}\left(\frac{g}{\kappa \rho}\right) \bar{\lambda}^{n}(\rho)
$$

Thus we have immediately $g$ independent and $g$ dependent RGs.

One can now ask how are they different? What is the criterion? Well, let's think of the fixed points of the groups. How does one find them in the field theoretic formalism? One looks for zeros of the $\beta$ functions. The fixed points of (5.8-5.10) are fixed points of the $g$ dependent RG. Fixed points of scale transformations are found by looking for fixed points of the dimensionless couplings $\bar{\lambda}, \bar{t}=\frac{t(\rho)}{\rho^{2} \kappa^{2}}$ and the dimensionless field $\bar{\phi}^{\prime}=\frac{\bar{\phi}(\rho)}{(\rho \kappa)^{\frac{d}{2}-1}}$. For the $g$ independent RG the equation (5.11) is

$$
\rho \frac{d \bar{\lambda}(\rho)}{d \rho}=-(4-d) \bar{\lambda}(\rho)+a_{2}(0) \bar{\lambda}^{2}(\rho)+\sum_{n=3}^{\infty} a_{n}(0) \bar{\lambda}^{n}(\rho)
$$

The $a_{n}$ are all just numbers so one gets out a fixed point to lowest order $\bar{\lambda}^{*}=\frac{(4-d)}{a_{2}(0)}$ (the higher orders can be found in the standard fashion [40][41]). Remember that a fixed point of the RG does not just consist of a zero of one of the $\beta$ functions, but actually of all of them. In the present case this means one must also consider the equation for the running temperature/mass and the running magnetization. Using the renormalization condition (5.1) when, $g=0$, implies that $t(\rho)=0$ is a fixed point. One can formally solve (5.9) to get $t(\rho)=t \exp \int \gamma_{\phi^{2}}$. Now, $t(\rho)=0$ implies $t=0$ due to the multiplicative renormalizability of $t$. The $g$ independence of the normalization condition (5.2) implies that $t=T-T_{c}(0)$. Thus, when $\bar{\phi}=0$, the fixed point being found here is the non-trivial fixed point of the isotropic system. An unstable Gaussian fixed point at $T=T_{c}(0)$, and a stable Gaussian fixed point at $T=\infty$, are also accessible to this $g$ independent RG. Clearly no anisoropic fixed point is accessible.

Turning now to the $g$ dependent RG one might think naively that to lowest order $t(\rho)=0$ (i.e. $T=T_{c}(g)$ ) and $\bar{\lambda}(\rho)=\frac{(4-d)}{a_{2}\left(\frac{g}{\kappa \rho}\right)}$ is a fixed point. However, it is hardly a fixed point as the last condition defines a function of $\rho$ and therefore cannot be preserved under the RG flow. Let us change variables to the floating coupling $h=a_{2}\left(\frac{g}{\kappa \rho}\right) \bar{\lambda}(\rho)$, the $\beta$ function of which is

$$
\beta(h)=-\varepsilon\left(\frac{g}{\kappa \rho}\right) h(\rho)+h^{2}(\rho)+\sum_{n=3}^{\infty} \frac{a_{n}\left(\frac{g}{\kappa \rho}\right)}{\left(a_{2}\left(\frac{g}{\kappa \rho}\right)\right)^{2}} h^{n}(\rho)
$$


where $\varepsilon\left(\frac{g}{\kappa \rho}\right)=4-d-\frac{d \ln a_{2}}{d \ln \rho}$. To find the fixed points of the system one must remember that there is really one more $\beta$ function equation. The dimensionless anisotropy $\frac{g}{\kappa \rho}$ actually plays the role of a new coupling in the problem, $\bar{g}$. The flow equation for it is

$$
\rho \frac{d \bar{g}}{d \rho}=-\bar{g}
$$

The fixed points of all three equations are to lowest order (we omit consideration of the Gaussian fixed points)

$$
\bar{g}=0, \quad h=\varepsilon(0), \quad t(\bar{g}=0)=0
$$

and

$$
\bar{g}=\infty, \quad h=\varepsilon(\infty), \quad t(\bar{g}=\infty)=0
$$

The first one of course is the same fixed point accessible to the $g$ independent RG. The second one, however, is totally inaccessible to this RG. If under favourable circumstances one has a good idea of what the effective degrees of freedom associated with the $g=\infty$ fixed point are, then one might impose normalization conditions at $g=\infty$. Once again the consequent RG would be $g$ independent, only this time the fixed point accessed would be (5.15) not (5.14) as in the case of the $g=0$ RG.

The question naturally arises as to the role of the minimally subtracted RG, which is loosely related to the field theoretic $\varepsilon$ expansion. It is used quite ubiquitously in particle physics. The answer is that it is the RG associated with the $g=0, t=0$ fixed point. It may be used to assist in defining the microscopic theory, in a fashion that is independent of an UV cutoff. However, in reality this fixed point plays no more preferred role than any of the others. Our formulation makes this manifest.

The RG that is capable of encapsulating both fixed points is the $g$ dependent RG. It is of course by no means unique. Just as in the non-crossover case where different renormalization schemes can exhibit the same physics, e.g. minimal subtraction and massless normalization conditions, so here there will be different schemes. The key is that they will differ only by relatively unimportant reparametrizations of the crossover variables. These differences are the field theoretic analog of the differences between block spinning, decimation, blockspinning on different lattices etc. in the Wilson type RG. In the space of coupling constants, $t, h$ and $g$, the physical system exhibits many points of scale invariance, two of which are (5.14) and (5.15). One of these is accessible to a $g=0 \mathrm{RG}$, one to a $g=\infty \mathrm{RG}$, and only for a $g$ dependent RG are both accessible. In this sense the $g$ dependent RG is global in the space of couplings whereas the others aren't. The reader should understand that we are not saying that using a $g$ independent RG precludes accessing the other fixed point, it is rather that the $g$ independent RG on its own cannot access it, i.e. the points of scale invariance and the fixed points of the $g$ independent RG are not the same. If one is able to supplement the $g$ independent RG with extra information then perhaps the other fixed point could become accessible. Almost certainly this will require the extra information to be non-perturbative with respect to the $g=0$ fixed point.

\section{$\S \S 5.2$ "Running" the Environment}

In the last section we considered RG flow generated by mass/temperature or momentum. Here we will consider briefly the concept of RG flows generated by the environment itself. Previously the environment was held fixed as far as the RG flow was concerned. One could examine what happened when one changed the environment by examining RG flows 
with respect to this new changed environment, one could not easily however go directly from one environment to another by the action of the flow itself. For the generic anisotropy $g$ this means that a particular value of $g$ fixes a curve, one then examines RG flow along this curve. Another value of $g$ yields another, distinct curve along which one can also examine RG flows. It is possible however to also generate an RG flow in the "direction" of the environment itself. This can generically be done by implementing normalization conditions with respect to some "fiducial" environment. We will assume that the parameter describing the environment does not itself renormalize and therefore there is no distinction between bare and renormalized $g$. For example consider the normalization conditions

$$
\begin{gathered}
\Gamma^{(2)}\left(p=0, t, \lambda, g=g_{0}\right)=t \\
\left.\frac{\partial \Gamma^{(2)}}{\partial p^{2}}\left(p, t, \lambda, g=g_{0}\right)\right|_{p=0}=1 \\
\Gamma^{(4)}\left(p=0, t, \lambda, g=g_{0}\right)=\lambda \\
\Gamma^{(2,1)}\left(p=0, t, \lambda, g=g_{0}, \kappa\right)=1
\end{gathered}
$$

The fiducial environment, $g_{0}$, is abitrary therefore the bare vertex functions must be independent of $g_{0}$. Thus

$$
\left(g_{0} \frac{\partial}{\partial g_{0}}+\beta\left(\lambda, \frac{g_{0}}{|t|^{\frac{1}{2}}}\right) \frac{\partial}{\partial \lambda}+\gamma_{\phi^{2}}\left(\lambda, \frac{g_{0}}{|t|^{\frac{1}{2}}}\right) t \frac{\partial}{\partial t}-\frac{1}{2} \gamma_{\phi}\left(\lambda, \frac{g_{0}}{|t|^{\frac{1}{2}}}\right)\left(N+\bar{\phi} \frac{\partial}{\partial \bar{\phi}}\right)\right) \Gamma^{(N)}=0
$$

where the Wilson functions $\beta, \gamma_{\phi}$ and $\gamma_{\phi^{2}}$ depend on $\lambda$ and $g_{0} /|t|^{\frac{1}{2}}$. The absence of a term $\gamma_{g} g \frac{\partial}{\partial g}$ is accounted for by the non-renormalization of $g$. More generally such a term would be necessary.

The RG equation can be solved in the standard manner using the method of characteristics to yield

$$
\begin{gathered}
\Gamma^{(N)}\left(p_{i}, t, \bar{\phi}, \lambda, g, g_{0}\right)=\left(g_{0} \rho\right)^{d-N \frac{(d-2)}{2}} \exp \left(-\frac{N}{2} \int_{1}^{\rho} \gamma_{\phi}\left(\lambda(x), \frac{g_{0} x}{|t|^{\frac{1}{2}}}\right) \frac{d x}{x}\right) \\
\Gamma^{(N)}\left(\frac{p_{i}}{\rho g_{0}}, \frac{t(\rho)}{\left(\rho g_{0}\right)^{2}}, \frac{\lambda(\rho) \bar{\phi}^{2}(\rho)}{2\left(\rho g_{0}\right)^{2}}, \lambda(\rho), \frac{g}{g_{0} \rho}, 1\right)
\end{gathered}
$$

where $t(\rho), \lambda(\rho)$, and $\bar{\phi}(\rho)$; the running temperature, coupling and magnetization respectively, satisfy

$$
\begin{gathered}
\rho \frac{d \bar{\lambda}(\rho)}{d \rho}=\beta\left(\lambda(\rho), \frac{g_{0} \rho}{|t|^{\frac{1}{2}}}\right) \\
\rho \frac{d t(\rho)}{d \rho}=\gamma_{\phi^{2}}\left(\lambda(\rho), \frac{g_{0} \rho}{|t|^{\frac{1}{2}}}\right) t(\rho) \\
\rho \frac{d \bar{\phi}(\rho)}{d \rho}=-\frac{1}{2} \gamma_{\phi}\left(\lambda(\rho), \frac{g_{0} \rho}{|t|^{\frac{1}{2}}}\right) \bar{\phi}(\rho)
\end{gathered}
$$


It should be clear here that in this RG, as distinct from that of the last section, it is the environment itself that is running. One would implement the same RG strategy however, i.e. use the arbitrariness in the scale $\rho$ to choose a value that allows for a perturbative evaluation of the right hand side of (5.21). The appropriate condition to set is $g=g_{0} \rho$. The beauty of running the environment is that, by an appropriate choice of initial condition for the characteristic equations, one can relate parameters associated with the environment $g$ to those associated with the environment $g=0$. Thus one can answer questions about the "shift" in a perturbatively controllable manner using RG techniques. It should also be clear that we could combine the two RG's and run the environment and mass/temperature together. We will return to these important matters in a future publication.

\section{$\S$ 6. FORMAL SCALING FORMS}

In this section, working still with an abstract anisotropy mass scale $g$, we will investigate the formal consequences of using a $g$ dependent RG. Consider first the solution (5.7) of the RG equation (5.6), where we work now with the floating coupling directly

$$
\begin{aligned}
\Gamma^{(N)}(t, \bar{\phi}, h, g, \kappa) & =(\kappa \rho)^{N+d-\frac{1}{2} N d} \\
& \exp \left(-\frac{N}{2} \int_{1}^{\rho} \gamma_{\phi}\left(h\left(x, \frac{g}{x}\right) \frac{d x}{x}\right) \Gamma^{(N)}\left(\frac{t(\rho)}{(\kappa \rho)^{2}}, \frac{\lambda \bar{\phi}^{2}(\rho)}{2(\kappa \rho)^{2}}, h(\rho), \frac{g}{\kappa \rho}, 1\right)\right.
\end{aligned}
$$

RG invariance implies that the right hand side of (6.1) is independent of $\rho$, therefore we are at liberty to choose a value of it that suits us. We will fix it using the condition

$$
t\left(\rho_{c}\right)=\rho_{c}^{2} \kappa^{2}
$$

$\rho_{c}$ being the particular value of the RG running scale which satisfies this equation. With the generic conditions (2.7) and (2.8), when $\bar{\phi}=0$, one finds $\rho^{2} \kappa^{2}=\xi^{-2}$, $\xi$ being the correlation length. We must then ask which correlation length, as this is sensitive once again to what normalization conditions are used. $g$ independent conditions would lead to the isotropic correlation length. Here we will assume that $g$ dependent renormalization is used and therefore the appropriate correlation length is $\xi_{g t}$, where $t=T-T_{c}(g)$. We append a $t$ to it also because of the assumption that $\bar{\phi}=0$ in the normalization conditions, i.e we are using the correlation length in the symmetric (disordered) phase.

We could have used other conditions to determine $\rho_{c}$ such as

$$
\begin{gathered}
\frac{\lambda\left(\rho_{c}\right)}{2} \bar{\phi}^{2}\left(\rho_{c}\right)=\rho_{c}^{2} \kappa^{2} \\
t\left(\rho_{c}\right)+\frac{\lambda\left(\rho_{c}\right)}{2} \bar{\phi}^{2}\left(\rho_{c}\right)=\rho_{c}^{2} \kappa^{2}
\end{gathered}
$$

The former with appropriate normalization conditions for $\Gamma^{(2)}$ and $\frac{\partial \Gamma^{(2)}}{\partial p^{2}}$ corresponds to a parametrization in terms of $\xi_{g \bar{\phi}}$, the correlation length in the ordered phase when $T=T_{c}(g)$. If $g$ independent renormalization was used then the corresponding correlation length would be that in the ordered phase of the isotropic system at $T=T_{c}(0)$. The condition (6.4) would yield a parametrization in terms of the "true" correlation length of the system, either above or below $T_{c}(g)$. From a purely formal standpoint any of the correlation lengths will do, they all correspond to non-linear scaling fields, however, as we shall see, a particular 
correlation length is naturally associated with a particular field theoretic RG, and within that RG certain fixed points of the system might be inaccessible, hence the utility of that correlation length from an RG standpoint is diminished. The optimum correlation length to use should be the true physical correlation length in the system which is associated with the RG that depends on all environmental parameters. Naturally, working with this correlation length might computationally be quite difficult. One is always at liberty to omit a parameter from the renormalization to try to simplify matters, however, by so doing one risks making a particular crossover in $\mathcal{M}$ inaccessible. Generically a breakdown in perturbation theory will then take place, indicating that one is trying to access a region of $\mathcal{M}$ which is inaccessible to the particular perturbation theory one is implementing.

An extremely useful way to parametrize a crossver is via the introduction of effective critical exponents [14], which are natural generalizations of the standard critical exponents associated with one particular fixed point. We define the effective critical exponent for the correlation length

$$
\nu_{e f f}=-\left.\frac{d \ln \xi_{g t}}{d \ln |t|}\right|_{g}
$$

With the condition (6.2) one sees that

$$
\nu_{e f f}=\frac{1}{2-\gamma_{\phi^{2}}}
$$

One can also define an effective exponent for the susceptibility

$$
\gamma_{e f f}=\left.\frac{d \ln \chi^{-1}}{d \ln |t|}\right|_{g}
$$

where $\chi^{-1}=\Gamma^{(2)}(t, \bar{\phi}, h, g, \kappa)$ is the inverse susceptibility. An effective exponent $\eta_{\text {eff }}$ is defined naturally via

$$
\eta_{e f f}=2-\left.2 \frac{d \ln \Gamma^{(2)}}{d \ln p^{2}}\right|_{t=0, g}
$$

which is equivalent via the condition (2.8) to $\eta_{\text {eff }}=\gamma_{\phi}(g / p)$. This $\gamma_{\phi}$, however, is exactly the same one that appears in (5.10), only its argument is different. For the exponents $\delta$ and $\beta$ associated with the ordered phase one can define effective exponents [29]

$$
\delta_{e f f}=\left.\frac{d \ln H}{d \ln \bar{\phi}}\right|_{t=0, g}
$$

and

$$
\beta_{e f f}=\left.\frac{d \ln \bar{\phi}}{d \ln |t|}\right|_{g}
$$

the latter being defined on the crossover coexistence curve. Finally, one can introduce an effective exponent for the specific heat

$$
\alpha_{e f f}=\left.\frac{d \ln \Gamma^{(0,2)}}{d \ln |t|}\right|_{g}
$$


There is one last concept we need before proceeding further. Consider in fixed dimension $d$, without $g$ dependence, how $\Gamma^{(4)}$ varies with temperature near a fixed point

$$
\frac{d \ln \Gamma^{(4)}}{d \ln |t|}=(4-d-2 \eta) \nu
$$

We use here the scaling behaviour of the four point coupling to get information about the dimensionality $d$ of the system. One can introduce the concept of an effective dimensionality, $d_{e f f}$, through the natural analog of (6.11)

$$
\left.\frac{d \ln \Gamma^{(4)}}{d \ln |t|}\right|_{g}=\left(4-d_{e f f}-2 \eta_{e f f}\right) \nu_{e f f}
$$

$d_{\text {eff }}$ is in fact simply related to $\gamma_{\lambda}, d_{\text {eff }}=4-\gamma_{\lambda}$, and can therefore be thought of as a measure of how important the leading irrelevant operator $\phi^{4}$ is. In the vicinity of a particular fixed point $\gamma_{\lambda}$ plays very little role, merely governing the corrections to scaling about that fixed point. In a small neighbourhood of this fixed point these are negligible. In the case of a crossover this will not be true as it is the corrections to scaling that actually interpolate from one fixed point to another. This is why $\gamma_{\lambda}$ takes on an important role. In fact, for crossovers where the different asymptotic regimes correspond to systems with different upper critical dimensions, $d_{\text {eff }}$, in the absence of transients, can correctly give the change in upper critical dimension as one interpolates between the two fixed points.

From the $g$ dependent RG equations for the various vertex functions one can derive relations between these effective exponents:

$$
\begin{gathered}
\gamma_{e f f}=\nu_{e f f}\left(2-\eta_{e f f}\right) \\
\delta_{e f f}=\left(\frac{d_{e f f}+2-\eta_{e f f}}{d_{e f f}-2+\eta_{e f f}}\right) \\
\beta_{e f f}=\frac{\nu_{e f f}}{2}\left(d_{e f f}-2+\eta_{e f f}\right) \\
\alpha_{e f f}=2-\nu_{e f f} d_{e f f}
\end{gathered}
$$

and combinations thereof. (RG derivations of these relations can be found in [28], [18] and [29]. As long as $g \ll \kappa$ and $\xi \gg \kappa^{-1}$, where $\kappa$ is a typical microscopic scale, these exponent relations will be universal, below the upper critical dimension. Just as in the standard case where the scaling relations imply that there are really only two independent critical exponents, so here there are really only two independent effective exponents. The difference with the crossovers considered here is that it is necessary to know one more function, $\gamma_{\lambda}=4-d_{e f f}$, which represents the effects of the leading irrelevant operator. In the case of a crossover for which the dimensionality does not change, e.g. crossover in a bicritical system, the effects of the leading irrelevant operator can be subsumed into an $N_{\text {eff }}$, which is a measure of the effective number of components of the order parameter [18].

Utilizing (6.2) in (6.1) yields

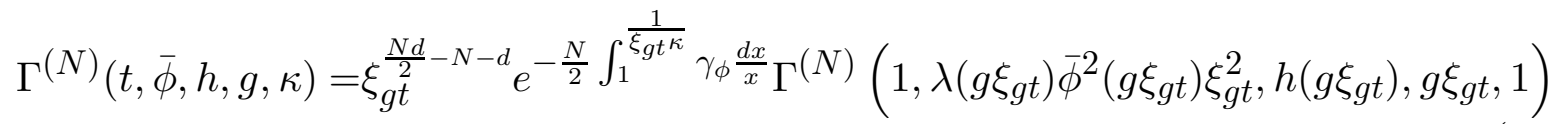


where using the running equations (5.8) and (5.10)

$$
\lambda\left(g \xi_{g t}\right) \bar{\phi}^{2}\left(g \xi_{g t}\right)=\lambda \bar{\phi}^{2} \exp \int_{1}^{\frac{1}{\kappa \xi_{g t}}}\left(\gamma_{\lambda}-\gamma_{\phi}\right) \frac{d x}{x}
$$

We can rewrite (6.17) in terms of the effective exponents

$$
\Gamma^{(N)}=\xi_{g t}^{\frac{N d}{2}-N-d} e^{-\frac{N}{2} \int_{\kappa}^{\xi_{g t}^{-1}} \eta_{e f f} \frac{d x}{x}} \mathcal{F}_{t}^{(N)}\left(\bar{\phi}^{2} e^{-\int_{\kappa}^{\xi_{g t}^{-1}}\left(d_{e f f}-2+\eta_{e f f}\right) \frac{d x}{x}}, g \xi_{g t}\right)
$$

where $\mathcal{F}_{t}^{(N)}$ is a universal function. Similarly, in terms of $\xi_{g \bar{\phi}}$ one finds

$$
\Gamma^{(N)}=\xi_{g \bar{\phi}}^{\frac{N d}{2}-N-d} e^{-\frac{N}{2} \int_{\kappa}^{\xi_{g \bar{\phi}}^{-1}} \eta_{e f f} \frac{d x}{x}} \mathcal{F}_{\bar{\phi}}^{(N)}\left(t e^{-\int_{\kappa}^{\xi_{g \bar{\phi}}^{-1}} \frac{1}{\nu_{e f f}} \frac{d x}{x}}, g \xi_{g \bar{\phi}}\right)
$$

In the various integrals in (6.19) and (6.20) one can change variables via the exponent definitions, exponent laws, and the conditions fixing $\rho$. For example using (6.5) and (6.6) one finds

$$
\int_{\kappa}^{\xi_{g t}^{-1}}\left(d_{e f f}-2+\eta_{e f f}\right) \frac{d x}{x}=\int_{\kappa}^{t}\left(d_{e f f}-2+\eta_{e f f}\right) \nu_{e f f} \frac{d t^{\prime}}{t^{\prime}}
$$

Thus

$$
\Gamma^{(N)}=e^{\int_{1}^{t}\left(N+d-\frac{N}{2}\left(d+\eta_{e f f}\right)\right) \nu_{e f f} \frac{d t^{\prime}}{t^{\prime}}} \mathcal{F}_{t}^{(N)}\left(\bar{\phi} e^{-\int_{1}^{t} \beta_{e f f} \frac{d t^{\prime}}{t^{\prime}}}, g e^{-\int_{1}^{t} \nu_{e f f} \frac{d t^{\prime}}{t^{\prime}}}\right)
$$

Similarly, one can also find that

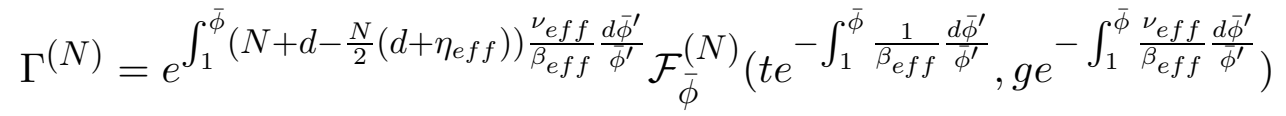

A particularly interesting case is the equation of state which can be written as

$$
H=\exp \left(\int_{1}^{\bar{\phi}} \delta_{e f f} \frac{d \bar{\phi}^{\prime}}{\bar{\phi}^{\prime}}\right) \mathcal{G}\left(t e^{-\int_{1}^{\bar{\phi}} \frac{1}{\beta_{e f f}} \frac{d \bar{\phi}^{\prime}}{\phi^{\prime}}}, g e^{-\int_{1}^{\bar{\phi}} \frac{\nu_{e f f}}{\beta_{e f f}} \frac{d \bar{\phi}^{\prime}}{\phi^{\prime}}}\right)
$$

where $\mathcal{G}$ is a universal function. The crossover coexistence curve is

$$
t=\exp \left(\int_{1}^{\bar{\phi}} \frac{1}{\beta_{\text {eff }}} \frac{d \bar{\phi}^{\prime}}{\bar{\phi}^{\prime}}\right)
$$

Thus using the effective exponents one can derive natural non-linear scaling fields (we use the term non-linear here to refer to the fact that they are non-linearly related to the linear scaling fields associated with the individual fixed points not as in the sense of Wegner that they are eigenfunctions of the dilatation operator). The natural non-linear scaling fields that appear above are the various correlation lengths, $\xi_{g t}=\exp \int_{1}^{t} \nu_{e f f}(x) \frac{d x}{x}, \xi_{g \bar{\phi}}$, 
etc. and $g$ (these are also non-linear scaling fields in the sense of Wegner). Note that the non-linear scaling field $\xi_{g t}$, interpolates between the linear scaling fields $t^{-\nu(0)}$ and $t^{-\nu(\infty)}$ associated with the $g=0$ and $g=\infty$ fixed points in the limits $g \rightarrow 0, \xi_{g t} \rightarrow \infty\left(g \xi_{g t} \rightarrow 0\right)$ and $g \xi_{g t} \rightarrow \infty$ respectively. In the equation of state there is another non-linear scaling field $t e^{-\int_{1}^{\bar{\phi}} \frac{1}{\beta_{e f f}} \frac{d \bar{\phi}^{\prime}}{\phi^{\prime}}}$ which interpolates between the two linear scaling fields $\frac{t}{\phi^{1 / \beta(0)}}$ and $\frac{t}{\phi^{1 / \beta(\infty)}}$ in the limits $g \rightarrow 0, \xi_{\bar{\phi}} \rightarrow \infty\left(g \xi_{\bar{\phi}} \rightarrow 0\right)$ and $g \xi_{\bar{\phi}} \rightarrow \infty$ respectively.

We hope the general pattern is clear. The natural non-linear scaling fields for the crossover can be found by taking the natural linear scaling fields for one of the fixed points, writing it in exponential form, e.g. $t^{\nu}=e^{\int_{1}^{t} \nu \frac{d x}{x}}$, then replacing the exponent with the effective one, e.g. $t^{\nu} \rightarrow e^{\int_{1}^{t} \nu_{e f f}}$. Note the effective exponent cannot be taken outside the integral to recover a form $t^{\nu_{e f f}}$. In this way one also arrives at the concept of effective crossover exponents. Standardly a crossover exponent involves the ratio of two eigenvalues of the RG operator linearized around the isotropic fixed point. The generalization of this involves exponentiating the integral of the difference of two effective exponents. Naturally, one must derive what these effective exponents are. This will be the task of most of the rest of the paper. The key is that they should be evaluated using an appropriate RG, i.e. one that is appropriate to the crossover in question - one that is environmentally friendly!

Finally, in this section we would like to discuss universality in the context of crossover behaviour. We have stated that in the regime where all length scales are much bigger than the lattice spacing $a$, that the scaling functions $\mathcal{F}^{(N)}, \mathcal{G}$, etc. and the effective exponents will all be universal functions. The meaning of universality here is just the standard one. For example, two layered Ising models with different lattice structures will exhibit precisely the same crossover curves (up to a trivial constant rescaling) as long as the finite size of the system $L \gg a$. However, the crossover curves for a layered Ising model of size $L$ but with different boundary conditions for instance will not be the same. Neither can they be made the same by any rescaling.

The environment affects the infrared behaviour of the theory and therefore the universality class. Universality in the non-crossover case is generically governed only by the dimensionality of the system and the symmetry of the order parameter. The environment gives us extra "labels" for delineating different crossover universality classes. The label "boundary condition" for instance allows us to classify, say, three dimensional Ising models with one finite dimension into different classes. The effective exponents are different for different crossover universality classes. However, the effective exponent laws have a much wider universality, being valid irrespective of crossover universality class. For example, the effective exponents obey scaling laws even when one of the fixed points is associated with the upper critical dimension. Logarithmic corrections to scaling are naturally incorporated in the effective exponents, as indeed are power law corrections coming from the leading irrelevant operator. Furthermore, in the simplified context of crossover from the Wilson-Fisher to Gaussian fixed points it was found [42] that effective exponents obeyed "all the thermodynamic scaling relations (e.g., $\alpha_{\text {eff }}+2 \beta_{\text {eff }}+\gamma_{\text {eff }}=2$ ) but not those of hyperscaling". In fact if one generalizes $d$ in this crossover to $4-\gamma_{\lambda}$, we find that all scaling laws including hyperscaling are obeyed by the effective exponents. Clearly the notion of $d_{e f f}$ is inappropriate here, however, the effects of the leading irrelevant operator must still be accounted for. This is achieved by the incorporation of $\gamma_{\lambda}$ into the scaling laws as above. In the context of the general type of crossover discussed in this paper we have seen that the scaling laws are always obeyed.

We are making an issue of these considerations here for the following reason: often it 
is the case that the asymptotic scaling behaviour at both ends of a crossover is known, i.e. the fixed points and critical exponents at either end are known. Given this knowledge Riedel and Wegner [14] wrote down Wilson functions based purely on the requirement that the function should allow for an interpolation between the two known fixed points. In light of our discussion of universality we regard this as a potentially dangerous procedure. To understand exactly why consider the Gaussian fixed point and the Wilson-Fisher fixed point for a three dimensional Ising model. These fixed points are the extremal fixed points for several crossover systems: three dimensional uniaxial dipolar ferromagnets, three dimensional Ising model in a transverse magnetic field, four dimensional layered Ising model (with possible different boundary conditions) as well as the standard Gaussian $\rightarrow$ Wilson-Fisher crossover of a three dimensional Ising model. If one adopts the approach of constructing a crossover curve by simply demanding that the two fixed points are captured in the RG flow then there is no way of distinguishing all these physically different crossovers. They are induced by different environmental variables which enter the theory in different ways. Only via an environmentally friendly RG can one distinguish the different behaviours of these systems and see whether they fall into different crossover universality classes.

In the context of a Wilsonian RG, such as momentum shell integration it is quite possible to obliterate real distinctions between crossover curves by implementing the momentum shell integrations using too rough an approximation, or by over emphasizing the high momenutum shells. For instance, Hertz [43] when considering the crossover between quantum and classical critical behaviour, derived approximate RG equations which were identical in form to those for the Gaussian Wilson-Fisher crossover, by ignoring the relevance of the gap in Matsubara frequencies relative to the momentum shell being integrated over, which difference is significant at the classical end of the crossover. As mentioned previously these two crossovers lie in two crossover universality classes.

The reader might indeed wonder if the concept of environment is so stringent as to make every crossover fall into its own universality class (apart from the standard trivial changes in lattice structure etc. one can engender). This is not so. For instance, in the above cited examples, it is known [44][43][8] that a four dimensional layered Ising model with periodic boundary conditions, and a three dimensional Ising model in transverse magnetic field, are equivalent. In the language used here they are in the same crossover universality class. We will show in section 11 that they ehhibit the same effective exponents.

\section{$\S$ 7. WHAT SHOULD WE PERTURB IN?}

In the last section we once again worked at a formal level, here we would like to discuss the explicit implementation of perturbation theory. We have explained that the main idea in crossovers is trying to quantitatively describe systems that exhibit different degrees of freedom at different scales. If one cannot solve a model exactly one must resort to an approximation procedure. Perturbation theory is a ubiquitous one. However, there is always the perennial question - perturbation theory in what? In applications of Wilsonian RGs this is often a very difficult question. In the field theory approach it looks much simpler, however, our discussion of the different field theoretic RGs should give one pause for thought. If one uses $g$ independent renormalization to eliminate UV problems then one discovers two things: firstly, that direct perturbation theory fails badly in the regime $g \xi \gg 1$. New "divergences" appear, which at a given order in perturbation theory are generically of the form $(g \xi)^{n}$ as $g \xi \rightarrow \infty$. Secondly, if one uses a $g$ independent RG one finds a running coupling which, in the same regime, becomes very strong. For example, for $\lambda \phi^{4}$ theory on $S^{1} \times R^{3}$ one finds

$$
\lambda(L \kappa)=\frac{\lambda}{1+\frac{3 \lambda}{16 \pi^{2}} \ln L \kappa}
$$


where $\kappa$ is the momentum/mass scale of interest. Clearly in the IR regime, $L \kappa \rightarrow 0$, one is entering a strongly coupled regime reminiscent of what occurs in QCD. The words "divergences" and "strong coupling" lead invariably to the invocation of "non-perturbative" techniques. It sometimes seems that what is meant by the latter is something which cannot be formulated perturbatively in terms of any coupling, such as doing a lattice simulation. We believe that quite often the case is overstated. To us, at least in many cases, "divergences" and "strong coupling" are symptomatic of the fact that one is implementing a perturbation theory which is not capturing the correct qualitative nature of the effective degrees of freedom. One of the main purposes of this paper is to show that more often than not these deficiences can be overcome perturbatively by finding an appropriate environment dependent expansion parameter.

In the case of a theory with an $O(N)$ symmetry, if one uses a $\frac{1}{N}$ expansion, one finds that if a $g$ independent renormalization is used, the $\frac{1}{N}$ expansion breaks down in the $g \xi \rightarrow \infty$ limit, except when $N \rightarrow \infty$ before $g \xi$ does. This is the spherical model limit, which is exactly solvable. One could also consider summing up sets of Feynman diagrams such as is done in summing up the daisy diagrams (Hartree/Fock approximation) in finite temperature field theory [45]. Naturally, this is always a tricky proposition as certain diagrams that are dominant in one regime might not be so dominant in another. One must still also address the question of how to renormalize. Additionally, one faces the problem that any crossover accessed by the resummation might not be the one of interest. This is in fact what happens in finite temperature field theory where the resummation of daisy diagrams in an attempt to get an improved description of a phase transition in a relativistic field theory merely accesses a mean field fixed point instead of the fixed point associated with the transition.

The key to understanding whether a particular perturbation parameter is suitable or not is to see how it gets dressed by fluctuations. If an effective coupling is small in one regime there is no guarantee that it will be small in some other. That is why one implements renormalization in the first place. One could consider a bare coupling to be small consistently if one was interested in physics at scales $\sim \Lambda$. However, when one is interested in scales $p \ll \Lambda$ one finds that the coupling gets large perturbative dressings, so one renormalizes to a point $\kappa \sim p$. In this case one has taken all the fluctuations between $\kappa$ and $\Lambda$, absorbed them into $\lambda_{B}$, and called it $\lambda_{\kappa}$, which is presumed to be a perfectly reasonable, finite, "observable" coupling. The best thing to do of course is consider an infinitessimal dressing — this yields the $\beta$ function, the fixed points of which correspond to those points where further dressing does not change the coupling. One of the main keys then to getting a reasonable perturbative parameter is having a good handle on the dressing of the coupling.

Let us now ask by what fluctuations the coupling is getting dressed? The fluctuations that represent the effective degrees of freedom at the scale of interest of course. Usually we do not have an exact representation of these fluctuations so we represent them perturbatively. Now, one might think it quite perverse to dress a renormalized parameter with fluctuations that were not a good representation of the effective degrees of freedom of the system, however, this is exactly what is commonly done, as if for example one uses a $g$ independent RG. Such a procedure is not terrible at all scales of course. When $\kappa \gg g$ the effective degrees of freedom are effectively $g$ independent and therefore for considering physics at these scales it would not be a bad approximation. The fluctuations are environment dependent and therefore the dressing of the coupling should be environment dependent. If one implements an environment dependent renormalization then one's effective expansion parameter is the running coupling which, for example, is a solution of (5.8) in the $g$ dependent case. The fixed points of (5.14) are (5.15), neither of these is a good 
expansion parameter throughout the crossover.

One is not guaranteed of course that the running coupling will be small throughout the crossover. This will in fact be the case in this paper where the coupling $\sim 1$ in certain asymptotic regimes. One could take the philosophy that only the lowest order terms should be believed in giving the solution, then the higher order terms are used merely to generate an iterated form of the lowest order solution. The preferable thing though is to use a resummation procedure. For our two loop results we use a [2,1] Padé approximant [40] to find

where

$$
\beta(h)=-\varepsilon(\bigcirc) h+\frac{h^{2}}{1+F h}
$$

$$
F=-\frac{4\left((5 N+22) A_{2(4-d)}\left(()-\frac{1}{2} \bigcirc^{2}\right)+(N+2) A_{2(4-d)} \phi\right)}{\left((N+8) A_{4-d} \bigcirc\right)^{2}}
$$

The explicit coupling obviously depends on the crossover in question. Naturally one would prefer to work to more than two loops, there is nothing in principle in our formalism besides tedious computation to prevent this from being done. So the idea then of implementing perturbation theory is the following: one first of all implements it in terms of the running coupling constant $h$ generated from a $g$ dependent RG. The actual $h$ used is then the resummed solution of the $\beta$ function equation. It is important to realize that in the case of the crossover this will be a function, not a pure number, though for a given $\rho$ and $g$, which can be arbitrary, it is just a particular number.

So, what other subtleties does the RG engender? Consider the solution of the RG equation (2.16) for $\Gamma^{(2)}$ in the disordered phase

$$
\Gamma^{(2)}(t, h, g, \kappa)=(\rho \kappa)^{2} e^{-\int_{1}^{\rho} \gamma_{\phi}(x) \frac{d x}{x}} \Gamma^{(2)}\left(\frac{t(\rho)}{\rho^{2} \kappa^{2}}, h(\rho), \frac{g}{\rho \kappa}, 1\right)
$$

Just because one can derive a coupling constant $h(\rho)$ that is not too badly behaved through the crossover does not imply that a perturbative expansion of $\Gamma^{(2)}$ will be sensible, the reason being that $h(\rho)$ is only one component in the expansion. $h(\rho)$ multiplies functions of $\frac{g}{\rho \kappa}$ and $\frac{t(\rho)}{\rho^{2} \kappa^{2}}$. Even if $h$ is not too large these functions may become large in certain physical regimes, e.g. $\frac{t}{g^{2}} \rightarrow 0$. If this is the case (and this will be the case in crossovers) then one must invoke the full power of the RG by using the arbitrariness in the renormalization scale $\rho$ to find a point $\rho_{c}$ where the functions are not large. If at this point one also finds that $h\left(\rho_{c}\right)$ is not too large then one can make real progress. Obviously $\rho_{c}$ will be explicitly $g$ dependent. The value of $\rho_{c}$ chosen will usually be determined by the fact that one is interested in a regime where the correlation length is large relative to $g^{-1}$ say. In such a regime perturbation theory breaks down in a fairly generic fashion. The thing to do, as is well known, is to map, using the freedom of choice in the renormalization scale, to a correlation length that is well out of the critical regime. What correlation length must one map? We discussed this matter somewhat in the last section. If one uses a $g$ independent RG then the natural correlation length is $\xi_{0}$, i.e. the correlation length in the isotropic system. Using such an $\mathrm{RG}$, however, will make certain regions of $\mathcal{M}$ inaccessible. The better correlation length to use is the physical one, naturally associated with the $g$ dependent $\mathrm{RG}$, which is global and can access all the fixed points of $\mathcal{M}$. A good mapping point, for instance, would be determined by $t\left(\rho_{c}\right)=\rho_{c}^{2} \kappa^{2}$, whereupon $\rho_{c}$ satisfies

$$
\rho_{c}^{2}=\frac{t}{\kappa^{2}} e^{\int_{1}^{\rho_{c}} \gamma_{\phi^{2}}(x) \frac{d x}{x}}
$$


where $t=\left(T-T_{c}(g)\right)$, i.e a $g$ dependent renormalization has been used. It is this key equation which must be solved perturbatively.

What do we mean by solving it perturbatively? Ultimately what one is expanding perturbatively are the characteristic functions of the RG, in particular the quantities $\gamma_{\phi^{2}}$, $\gamma_{\phi}$ and $\gamma_{\lambda}$. One determines them as series in the solution of the resummed $\beta$ function differential equation. One should in fact, based on a democratic treatment of all the characteristic functions, Padé resum the series for $\gamma_{\phi^{2}}$ and $\gamma_{\phi}$ as well. To two loops, obviously $\gamma_{\phi}$ cannot be resummed. The $[2,1]$ Padé resummed expression for $\gamma_{\phi^{2}}$ is

$$
\gamma_{\phi^{2}}=\left(\frac{N+2}{N+8}\right) \frac{h}{1-\frac{6}{(N+8)}\left(\frac{\left.A_{2(4-d)}(\bigvee)-\frac{1}{2} \bigcirc^{2}\right)}{\left(A_{4-d} \bigcirc\right)^{2}}+\frac{1}{3} \frac{A_{2(4-d)} \phi}{\left(A_{4-d} \bigcirc\right)^{2}}\right) h}
$$

For small values of $N$ one finds that the effective exponents derived from [2,1] Padé resummed Wilson functions differ little from those derived from a using resummed $\beta$-function for the floating coupling with the perturbative series for the effective exponents in this coupling. See Fig.10 for an illustration of this in the context of dimensional crossover. The former prescription caprures the exactly solvable $n=\infty$ limit and therefore seems prefered. Correlation functions are related to exponentials of the Wilson functions, it would be quite against the spirit of the RG to start perturbatively expanding the exponentials, otherwise there would be little point in using the RG in the first place. This is one of the problems with trying to use $\varepsilon$ expansion methods to calculate scaling functions. The question is always: if something is to a power of $\varepsilon$ should one $\varepsilon$ expand it? In (7.3) one should expand $\gamma_{\phi^{2}}$ in the integrand to a given order $h^{n}, h$ being the resummed solution of the $\beta$ function differential equation to order $h^{n+1}$. One should not expand the exponential. To lowest order for instance, one would solve

$$
\rho_{c}^{2}=\frac{t}{\kappa^{2}} \exp \left[\left(\frac{N+2}{N+8}\right) \int_{1}^{\rho_{c}} h\left(x, \frac{g}{x}\right) \frac{d x}{x}\right]
$$

where $h$ is a solution of

$$
\rho \frac{d h}{d \rho}=-\varepsilon(\bigcirc) h+h^{2}
$$

More often than not one will have to resort to numerical techniques to solve the equation that fixes $\rho$, however, we will see shortly that as long as one captures the essential physics of the crossover with an appropriate RG then one's final answers are rather robust under changes in solving the condition.

Besides the ambiguity in using the $\varepsilon$ expansion to determine scaling functions, i.e. should or shouldn't one expand something which is to a power of $\varepsilon$, there are two other defects. First of all, if one makes a definite choice in deciding to expand everything in $\varepsilon$, as is done in finding the equation of state [46], one will find that the resulting expression is only thermodynamically valid in a restricted region of the thermodynamic state space. Secondly, if one is considering a crossover wherein the upper critical dimension changes from one asymptotic region to another, then an $\varepsilon$ expansion will be inadequate, being able to capture the singularity at one end but totally failing to capture the singularity at the other. In principle, in such circumstances a $\frac{1}{N}$ expansion could work, if used in conjunction with an appropriate environmentally friendly renormalization. However, it is well known that the $\frac{1}{N}$ expansion gives poor results for small values of $N$, is difficult to implement at 
higher orders, and additionally such an expansion would be useless if there was a change in the number of symmetry components of the order parameter during the crossover, e.g. for the bicritical crossover. We believe our perturbation technique to be the only one on offer applicable to crossovers where both the dimensionality and/or the number of components of the order parameter can change.

In the above we are exploiting the arbitrariness in the RG scale to allow us to map to a point where perturbation theory can be trusted. We now discuss how one can exploit the various renormalization conditions in order to facilitate calculations. We have already seen that the type of condition used, in the sense of dependence or independence of the environment, is quite crucial to achieving a description of crossover systems. There are, as pointed out, different, inequivalent representations of the RG. Having determined the RG that is most suitable, and having determined the RG scale that allows perturbation theory to be implemented across the crossover, one can make further simplifications by trying to put as much of the physics as possible into the characteristic functions alone.

What this means is that for an object like the inverse susceptibility, choosing a renormalization condition like (5.2), implies

$$
\Gamma^{(2)}\left(1, h\left(\rho_{c}\right), \frac{g}{\rho_{c} \kappa}, 1\right)=1
$$

which gives

$$
\Gamma^{(2)}(t, h, g, \kappa)=\exp \int_{1}^{t}\left(\frac{2-\gamma_{\phi}}{2-\gamma_{\phi^{2}}}\right) \frac{d x}{x}
$$

Clearly through the choice of normalization condition all the physics has been put into the two functions $\gamma_{\phi}$ and $\gamma_{\phi^{2}}$; i.e. $\Gamma^{(2)}\left(1, h\left(\rho_{c}\right), \frac{g}{\rho_{c} \kappa}, 1\right)$ did not need to be perturbatively expanded. According to the framework outlined above, a perturbative treatment of (7.8) entails expanding $\beta, \gamma_{\phi}$ and $\gamma_{\phi^{2}}$ as a power series in $h$ to a certain order, Padé resumming the resulting series, and then putting in $h$ as the resummed solution of the $\beta$ function. We could have used a condition other than (7.7), such as

$$
\Gamma^{(2)}\left(0, h(\rho), \frac{g}{\rho \kappa}, 1\right)=0
$$

. In such circumstances $\Gamma^{(2)}\left(1, h\left(\rho_{c}\right), \frac{g}{\rho_{c} \kappa}, 1\right)$ would also be perturbatively expanded in $h$, hence not all the physics would be in the characteristic functions. One would obtain an expression of the form

$$
\Gamma^{(2)}(t, h, g, \kappa)=\exp \left(\int_{1}^{t}\left(\frac{2-\gamma_{\phi}}{2-\gamma_{\phi^{2}}}\right) \frac{d x}{x}\right)\left(1+h\left(\rho_{c}\right) B_{1}\left(\frac{g}{\kappa \rho_{c}}\right)+h^{2}\left(\rho_{c}\right) B_{2}\left(\frac{g}{\kappa \rho_{c}}\right)+\cdots\right)
$$

where $B_{1}$ and $B_{2}$ are dependent on the precise normalization condition one uses. For the massless normalization condition (7.9) $B_{1}$ contains a contribution from the difference between the massive and massless tadpole. At the one loop level we could have exploited the arbitrariness in the renormalization scale $\rho$ to set $\Gamma^{(2)}\left(1, h\left(\rho_{c}\right), \frac{g}{\rho_{c} \kappa}, 1\right)=1$. In this case $\gamma_{\phi^{2}}$ will not be the same as found using the condition (7.7). A quantity such as an effective exponent, however, will be invariant under such a change in normalization point. Unfortunately this would not work beyond the one loop level, for $d<4$, due to the presence of IR divergences in $B_{2}$, as was pointed out in section 4 . If one wanted to calculate a scaling 
function, such as the susceptibility above, the functions $B_{1}$ and $B_{2}$ would, for instance, carry information about the difference between the GMS method used in [15] and [19], and using a normalization condition, as we have used in our method. Although they might not be IR divergent, as emphasized above, this difference is not easily exponentiated, so that in the calculation of a scaling function GMS will yield less information than using normalization conditions. This is one disadvantage of GMS versus our method.

$\bar{\lambda}$

It is useful to give an analog expression diagramatically to lowest order in the coupling

$$
\Gamma^{(2)}(t, h, g, \kappa)=\exp \left(\int_{1}^{t}\left(1-\frac{(N+2)}{6}\left(A_{(4-d)} \bigcirc\right) \bar{\lambda}\right) \frac{d x}{x}\right)\left(1+\frac{\bar{\lambda}}{6}(N+2)\left(\bigcirc^{\prime}-\bigcirc\right)+\cdots\right)
$$

The quantity $\left(\bigcirc^{\prime}-\bigcirc\right.$ ) is a measure of what has been "left out" of the RG and would be the analog of $\ln \left(\frac{t}{\kappa^{2}}\right)$ in four dimensions, which is the difference to lowest order between the normalization condition $\Gamma^{(2)}(t)=t$ and minimal subtraction. The RG equation tells us how to perform an infinitessimal dressing of a quantity. By different choices of counterterm one can dress the quantity with different fluctuations, $g$ dependent, $g$ independent etc. Among the $g$ dependent schemes themselves though, as we are seeing here, one can dress things differently. Should one dress things using the whole of a Feynman diagram, or what we think to be the most important part of it, e.g. the mass independent part, or....? The RG only tells us about dressings, but because we can integrate an infinitessimal dressing, one can, in principle, derive a huge amount of information from it, as this integrated dressing gets exponentiated in the process of solving the RG equation. What is not included as dressing cannot be exponentiated, therefore one potentially loses a large amount of predictive power. Manifestly the best thing do do then is dress up as much as possible, and particularly in an environmentally friendly manner.

\section{$\S$ 8. THE FLOATING FIXED POINT}

In this section we will introduce the concept of the floating fixed point. We said in section 6 that to find the fixed points of the RG one had to find the fixed points of all the $\beta$ functions, including $\beta(\bar{g})$. We exhibited the two relevant fixed points. If we had just examined $\beta(\bar{\lambda})$ in isolation, and set $\beta(\bar{\lambda})=0$, to lowest order one finds $\bar{\lambda}=\frac{d-4}{\frac{(N+8)}{6}\left(A_{(4-d)} \bigcirc\right)}$. In the limit $\frac{g}{\rho \kappa} \rightarrow \infty$ one finds that $\bar{\lambda} \rightarrow 0$. This implies that it wasn't very useful to think of the algebraic zero of the $\beta$ function as a fixed point as it did not cross over between the right values (a more detailed discussion of this can be found in [27]). If we use the floating coupling, however, and consider $\beta(h)=0$, one finds that the solution of this equation interpolates between the correct fixed points of the crossover. The condition $\beta(h)=0$ defines the floating fixed point $h^{*}$. To lowest order $h^{*}=\varepsilon(\bigcirc)$. To higher order one could try to iterate a solution, i.e take this solution, put it into the $h^{3}$ term in $\beta(h)$, and resolve the algebraic equation $\beta(h)=0$. To the next order the iterative solution is

$$
h^{*}(z)=\varepsilon(z)+\frac{4}{(N+8)^{2}}\left((5 N+22) f_{1}(z)-(N+2) f_{2}\right) \varepsilon(z)^{2}+O\left(\varepsilon(z)^{3}\right)
$$

Finally one can Padé resum the floating fixed point to obtain

$$
h^{*}(z)=\frac{\varepsilon(z)}{1-\frac{4}{(N+8)^{2}}\left((5 N+22) f_{1}(z)-(N+2) f_{2}\right) \varepsilon(z)}
$$


The utility of using the floating fixed point is that to find it one need only solve an algebraic equation instead of a differential equation. The floating fixed point is guaranteed to give the correct asymptotic answers, i.e. the floating fixed point coincides at $\bar{g}=0$ and $\bar{g}=\infty$ with the isotropic and anisotropic fixed points which are the fixed points of the system of differential $\beta$ function equations. Naturally the solution of the differential equation and the algebraic equation will differ through the crossover. This difference is found (in all cases examined so far) to be relatively small, so the floating fixed point can be thought as giving a good approximation to the true crossover $h$.

One can go through the entire analysis of sections 6 and 7 expanding everything around the floating fixed point in analogy to expansion around a normal fixed point. For example, the non-linear scaling field $\xi_{g t}$ expanded around the floating fixed point becomes

$$
\xi_{g t}=A(t, g) \exp \left(-\int_{1}^{t} \nu_{e f f} * \frac{d t^{\prime}}{t^{\prime}}\right)
$$

where $A(t, g)=\exp \int_{1}^{t}\left(\nu_{e f f}{ }^{*}-\nu_{e f f}\right)$ is a correction to scaling factor around the floating fixed point and $\nu^{*}$ is the effective correlation length exponent evaluated at the floating fixed point. Remember that $\nu_{e f f}{ }^{*}$ is a function of $t$ and $g$, therefore one cannot pull it out of the integral as if it were a constant. This can only be done asymptotically where $\nu^{*}$ becomes the exponent at the true fixed points. If we had expanded around the isotropic fixed point we would find

$$
\xi_{g t}=B(t, g) \exp \left(-\int_{1}^{t} \nu(0) \frac{d t^{\prime}}{t^{\prime}}\right)
$$

where $B(t, g)=\exp \int_{1}^{t}\left(\nu(0)-\nu_{\text {eff }}\right)$ is a correction to scaling factor around the isotropic fixed point. The key difference is that $A$ is small throughout the crossover and approaches zero in the asymptotic regimes, whereas $B$ goes to zero near the isotropic fixed point but becomes singular near the anisotropic fixed point due to the fact that it must contain a singularity of the form $t^{-(\nu(\infty)-\nu(0))}$ in order that the isotropic singularity be replaced by the anisotropic one. Thus using the floating fixed point corrections to scaling can be made small throughout the crossover. Importantly, in our formalism the corrections to scaling around the floating fixed point can also be calculated explicitly. One can define effective exponents, $\alpha_{e f f}{ }^{*}, \beta_{e f f}{ }^{*}, \gamma_{e f f}{ }^{*}, \delta_{e f f}{ }^{*}, \nu_{e f f}{ }^{*}$ and an effective dimension $d_{\text {eff }}^{*}$ associated with the floating fixed point and find that they obey exact analogs of the equations (6.13-6.16). Similarly for all the non-linear scaling fields, there are floating fixed point analogs which differ from the one's already defined only by small corrections to scaling throughout the crossover.

\section{$\S$ 9. DIAGRAMMATIC REPRESENTATION OF EFFECTIVE EXPONENTS AND SCALING FIELDS}

In the last sections we tried to convey the utility of the field theoretic RG if implemented judiciously. In this section we wish to derive perturbative expressions for the effective exponents and some of the scaling fields mentioned in section 6 . Things will still be formal to the extent that we do not specialize yet to a particular crossover. The reason for doing this is that in our diagramatic notation it is clear that any crossover can be treated by putting into the diagrams the effects of the particular environmental parameters that are inducing that crossover, whatever they may be. We have already given the expression for 
the Wilson functions diagramatically so we just need to construct the effective exponents from them and $d_{e f f}$. Note that here we do not Padé resum $\gamma_{\phi^{2}}$ and we implement the scaling laws perturbatively in $h$. These expressions will be useful for comparative purposes and as we shall see are quite reliable for small values of $N$. Analogous expressions can be obtained when $\gamma_{\phi^{2}}$ is also resummed, in which case the effective exponents should be constructed from these resummed quantities without further expansion.

One finds to two loops

$$
\begin{gathered}
\eta_{\text {eff }}=2 \frac{(N+2)}{(N+8)^{2}} \frac{A_{2(4-d)} \phi}{\left(A_{4-d} \bigcirc\right)^{2}} h^{2} \\
\nu_{\text {eff }}=\frac{1}{2}+\frac{(N+2)}{4(N+8)} h+ \\
\quad \frac{(N+2)}{8(N+8)^{2}}\left(N+2+12 \frac{A_{2(4-d)}\left(()-\frac{1}{2} \bigcirc^{2}\right)}{\left(A_{4-d} \bigcirc\right)^{2}}+4 \frac{A_{2(4-d)} \phi}{\left(A_{4-d} \bigcirc\right)^{2}}\right) h^{2} \\
\left.\gamma_{\text {eff }}=1+\frac{(N+2)}{2(N+8)} h+\frac{(N+2)}{4(N+8)^{2}}\left(N+2+12 \frac{A_{2(4-d)}\left(()-\frac{1}{2} \bigcirc^{2}\right)}{\left(A_{4-d} \bigcirc\right)^{2}}\right)\right) h^{2}
\end{gathered}
$$

Using $d_{\text {eff }}=4-\varepsilon(\bigcirc)-\frac{\beta(h)}{h}$ one finds

$$
d_{e f f}=4-h-\frac{4}{(N+8)^{2}}\left((5 N+22) \frac{\left.A_{2(4-d)}(\downarrow)-\frac{1}{2} \bigcirc^{2}\right)}{\left(A_{4-d} \bigcirc\right)^{2}}+(N+2) \frac{A_{2(4-d)} \phi}{\left(A_{4-d} \bigcirc\right)^{2}}\right) h^{2}
$$

and hence

$$
\begin{gathered}
\alpha_{e f f}=\frac{(4-N)}{2(N+8)} h+\left(\frac{(4-N)(N+2)+16(N+8) \frac{A_{2(4-d)}\left(\mathbb{V}-\frac{1}{2} \bigcirc^{2}\right)}{\left(A_{4-d} \bigcirc\right)^{2}}}{4(N+8)^{2}}\right) h^{2} \\
\beta_{e f f}=\frac{1}{2}-\frac{3}{2(N+8)} h-\left(\frac{3(N+2)+2(7 N+38) \frac{\left.A_{2(4-d)}(\sqrt{ })-\frac{1}{2} \bigcirc^{2}\right)}{\left(A_{4-d} \bigcirc\right)^{2}}}{4(N+8)^{2}}\right) h^{2} \\
\delta_{\text {eff }}=3+h+\frac{1}{2}\left(1+8 \frac{(5 N+22)}{(N+8)^{2}} \frac{A_{2(4-d)}\left(\mathbb{V}-\frac{1}{2} \bigcirc^{2}\right)}{\left(A_{4-d} \bigcirc\right)^{2}}\right) h^{2}
\end{gathered}
$$

If one solves the Padé resummed differential equation (7.1) and inserts it in these expressions then one has the effective exponents for a general class of crossovers. For the Padé resummed floating fixed point

$$
h^{*}=\varepsilon(\bigcirc)\left(1+\frac{4}{(N+8)^{2}}\left((5 N+22) \frac{\left.A_{2(4-d)}(\bigcup)-\frac{1}{2} \bigcirc^{2}\right)}{\left(A_{4-d} \bigcirc\right)^{2}}+(N+2) \frac{A_{2(4-d)} \phi}{\left(A_{4-d} \bigcirc\right)^{2}}\right) \varepsilon(\bigcirc)\right)^{-1}
$$


Thus one finds

$$
\begin{aligned}
& \eta_{\text {eff }}{ }^{*}=2 \frac{(N+2)}{(N+8)^{2}} \frac{A_{2(4-d)} \phi}{\left(A_{4-d} \bigcirc\right)^{2}} \varepsilon(\bigcirc)^{2}\left(1+\frac{4}{(N+8)^{2}}\left({ }_{(5 N+22)} \frac{\left.A_{2(4-d)}(\downarrow)-\frac{1}{2} \bigcirc^{2}\right)}{\left(A_{4-d} \bigcirc\right)^{2}}+(N+2) \frac{A_{2(4-d)} \phi}{\left(A_{4-d} \bigcirc\right)^{2}}\right) \varepsilon(\bigcirc)\right)^{-2} \\
& \nu_{e f f}^{*}=\frac{1}{2}+\frac{(N+2)}{4(N+8)} \varepsilon(\bigcirc)\left(1+\frac{4}{(N+8)^{2}}\left({ }_{(5 N+22)} \frac{\left.A_{2(4-d)}(\bigcup)-\frac{1}{2} \bigcirc^{2}\right)}{\left(A_{4-d} \bigcirc\right)^{2}}+(N+2) \frac{A_{2(4-d)} \phi}{\left(A_{4-d} \bigcirc\right)^{2}}\right) \varepsilon(\bigcirc)\right)^{-1} \\
& +\frac{(N+2)}{8(N+8)^{2}}\left(N+2+12 \frac{\left.A_{2(4-d)}(\downarrow)-\frac{1}{2} \bigcirc^{2}\right)}{\left(A_{4-d} \bigcirc\right)^{2}}+4 \frac{A_{2(4-d)} \phi}{\left(A_{4-d} \bigcirc\right)^{2}}\right) \varepsilon(\bigcirc)^{2} \\
& \times\left(1+\frac{4}{(N+8)^{2}}\left({ }_{(5 N+22)} \frac{\left.A_{2(4-d)}(\backslash)-\frac{1}{2} \bigcirc^{2}\right)}{\left(A_{4-d} \bigcirc^{2}\right)^{2}}+(N+2) \frac{A_{2(4-d)} \phi}{\left(A_{4-d} \bigcirc^{2}\right)^{2}}\right) \varepsilon(\bigcirc)\right)^{-2}
\end{aligned}
$$

With the effective dimension at the floating fixed point, $d_{\text {eff }}^{*}=\varepsilon(\bigcirc)$, one can easily derive all the other effective exponents at the floating fixed point. We leave this as an exercise for the reader.

Consider now the non-linear scaling fields $\xi_{g t}=e^{-\int_{1}^{t} \nu_{e f f} \frac{d t^{\prime}}{t^{\prime}}}$ and $x=t e^{-\int_{1}^{\bar{\phi}} \frac{1}{\beta_{e f f}} \frac{d \bar{\phi}^{\prime}}{\phi^{\prime}}}$. Diagramatically

$$
\begin{aligned}
& \xi_{g t}= \\
& \exp \left(-\int_{1}^{t}\left(\frac{1}{2}+\frac{(N+2)}{4(N+8)} h+\frac{(N+2)}{8(N+8)^{2}}\left(N+2+12 \frac{\left.A_{2(4-d)}(\backslash)-\frac{1}{2} \bigcirc^{2}\right)}{\left(A_{4-d} \bigcirc\right)^{2}}+4 \frac{A_{2(4-d)} \emptyset}{\left(A_{4-d} \bigcirc\right)^{2}}\right) h^{2}\right) \frac{d t^{\prime}}{t^{\prime}}\right)
\end{aligned}
$$

and

$$
x=t \exp \left(-2 \int_{1}^{\bar{\phi}}\left(1+\frac{3 h}{(N+8)}+\left[3(N+12)+2(7 N+38) \frac{\left.A_{2(4-d)}(\bigcup)-\frac{1}{2} \bigcirc^{2}\right)}{\left(A_{4-d} \bigcirc^{2}\right)^{2}}\right] \frac{h^{2}}{2(N+8)^{2}}\right)\right)
$$

In terms of the Padé resummed floating fixed point

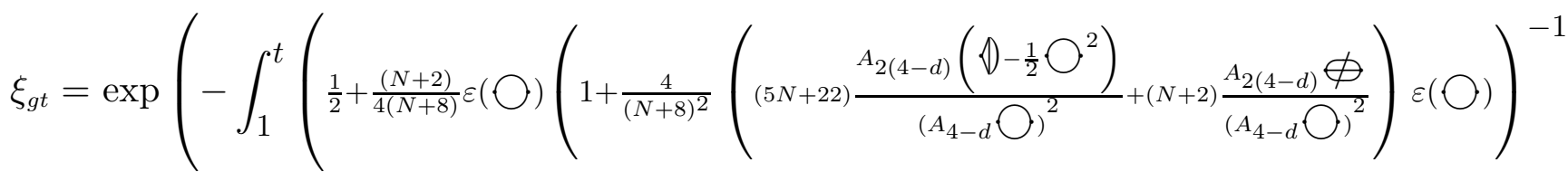

$$
\begin{aligned}
& +\frac{(N+2)}{8(N+8)^{2}}\left(N+2+12 \frac{A_{2(4-d)}\left(()_{-\frac{1}{2}} \bigcirc^{2}\right)}{\left(A_{4-d} \bigcirc\right)^{2}}+4 \frac{A_{2(4-d)} \phi}{\left(A_{4-d} \bigcirc\right)^{2}}\right) \varepsilon(\bigcirc)^{2} \times \\
& \left.\left.\left(1+\frac{4}{(N+8)^{2}}\left({ }_{(5 N+22)} \frac{\left.A_{2(4-d)}(\backslash)-\frac{1}{2} \bigcirc^{2}\right)}{\left(A_{4-d} \bigcirc\right)^{2}}+(N+2) \frac{A_{2(4-d)} \varnothing}{\left(A_{4-d} \bigcirc\right)^{2}}\right) \varepsilon(\bigcirc)\right)^{-2}\right) \frac{d t^{\prime}}{t^{\prime}}\right)
\end{aligned}
$$


and

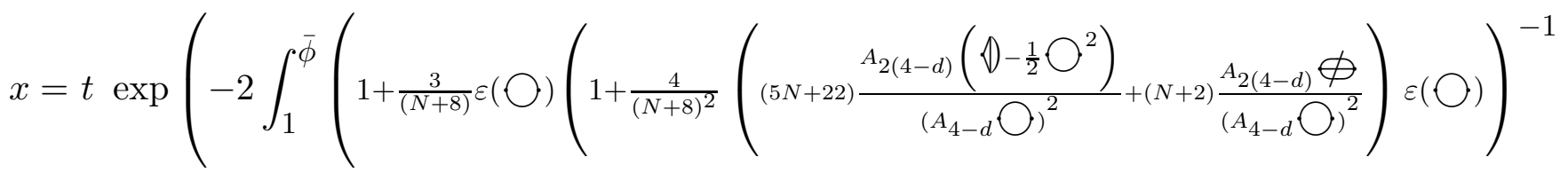

$$
\begin{aligned}
& +\frac{\varepsilon(\bigcirc)}{2(N+8)^{2}}\left(3(N+12)-2(7 N+38) \frac{\left.A_{2(4-d)}(\downarrow)-\frac{1}{2} \bigcirc^{2}\right)}{\left(A_{4-d} \bigcirc\right)^{2}}\right) \times \\
& \left.\left.\left(1+\frac{4}{(N+8)^{2}}\left({ }_{(5 N+22)} \frac{\left.A_{2(4-d)}(\downarrow)-\frac{1}{2} \bigcirc^{2}\right)}{\left(A_{4-d} \bigcirc\right)^{2}}-(N+2) \frac{A_{2(4-d)} \emptyset}{\left(A_{4-d} \bigcirc\right)^{2}}\right) \varepsilon(\bigcirc)\right)^{-2}\right)\right)
\end{aligned}
$$

It is impressive to see from these expressions just how much diagramatic information has been extracted using the RG. The reason we have exhibited these cumbersome looking expressions, is that they can serve as "black boxes" for evaluating crossover functions. For any crossover falling within the general class treated, in our two loop perturbative analysis, the reader may take the expressions given, insert the appropriate propagator into the diagrams, crank the handle, either numerically or analytically, and obtain the corresponding crossover scaling information. Now it is time to turn to a specific example of a crossover to show all the ideas and formalism we have presented at work.

\section{$\S$ 10. EXPLICIT DIMENSIONAL CROSSOVER RESULTS}

\section{$\S \S 10.1$ Periodic Boundary Conditions: One Loop Results}

The specific crossover we will consider here is that of a $d$ dimensional layered system satisfying periodic boundary conditions (or in particle physics language $\lambda \phi^{4}$ theory on $\left.R^{d-1} \times S^{1}\right)$. We consider, to begin with, an $O(N)$ vector model. We will be concerned here with $T>T_{c}(L)$, where $T_{c}(L)$ is the critical temperature of the finite size system, and $d \leq 4$. We will also assume here that the finite system does exhibit a critical point, though the formalism is equally applicable to the case where such does not exist. The environment here is the thickness of the system, which we denote by $L$, and the periodic boundary conditions. The results below are equally applicable to relativistic finite temperature after the trivial substitution $L=1 / T$ and $\xi_{L}=1 / m_{T}$.

Let us begin by examining the one loop expressions

$$
\begin{gathered}
\beta(h)=-\varepsilon(\rho \kappa L) h+h^{2}+O\left(h^{3}\right) \\
\gamma_{\phi^{2}}=\frac{(N+2)}{(N+8)} h+O\left(h^{2}\right)
\end{gathered}
$$

$\gamma_{\phi}=0$ to one loop. The solution of (10.1) is

$$
h(\kappa L \rho)=\frac{e^{-\int_{1}^{\rho} \varepsilon(\kappa L x) \frac{d x}{x}}}{h_{0}^{-1}-\int_{1}^{\rho} e^{-\int_{1}^{x} \varepsilon\left(\kappa L x^{\prime}\right) \frac{d x^{\prime}}{x^{\prime}}} \frac{d x}{x}}
$$


Choosing $\alpha_{1}=0, \alpha_{2}=\rho_{c}^{2}$, in our normalization schemes we have $\rho_{c} \kappa=\xi_{L}^{-1}$. Thus we have $h$ in terms of $z=L / \xi_{L}$, and an initial coupling at the scale $\kappa$, or equivalently $z_{0}=\kappa L$. Substituting this into the one loop contribution for $\gamma_{\phi^{2}}$ we have

$$
\gamma_{\phi^{2}}(z)=\frac{(N+2)}{(N+8)} \frac{e^{-\int_{z_{0}}^{z} \varepsilon(x) \frac{d x}{x}}}{h\left(z_{0}\right)^{-1}-\int_{z_{0}}^{z} e^{-\int_{z_{0}}^{x} \varepsilon(x) \frac{d x^{\prime}}{x^{\prime}}} \frac{d x}{x}}
$$

Now, in $d<4$ there is a non-trivial fixed point of the bulk system, and hence if we impose the initial condition on the RG flow at $z_{0}=\infty$, i.e. at zero correlation length, and choose a finite renormalized coupling $h(\infty)$, we will be probing the universal part of the crossover. This is not a necessity but merely allows us to isolate this universal part. Often, one is also interested in corrections to scaling, if $z_{0}$ is not chosen to be infinite then these are automatically included in the expressions, and as emphasized in section 7 the effective exponent scaling laws are still valid in this case. One finds

$$
h(z)=-\frac{A_{4-d} \bigcirc}{\bigcirc}
$$

which depends on only the variable $z$, and interpolates between the bulk and reduced fixed points. This is the equation of the separatrix between the two fixedpoints in the one loop approximation. It is not difficult to verify that (10.5) satisfies the one loop $\beta$ function equation. Note that the component diagrams are finite for $d<4$ but for $d=4 \bigcirc$ diverges, it is therefore impossible to eliminate the dependence on $z_{0}$ in this case.

With periodic boundary conditions we have

$$
\varepsilon(z)=5-d-(7-d) \frac{\sum_{n=-\infty}^{\infty} \frac{4 \pi^{2} n^{2}}{z^{2}}\left(1+\frac{4 \pi^{2} n^{2}}{z^{2}}\right)^{\frac{d-9}{2}}}{\sum_{n=-\infty}^{\infty}\left(1+\frac{4 \pi^{2} n^{2}}{z^{2}}\right)^{\frac{d-7}{2}}}
$$

and the universal one loop floating coupling

$$
h(z)=(5-d) \frac{\sum_{n=-\infty}^{\infty}\left(1+\left(\frac{2 \pi n}{z}\right)^{2}\right)^{\frac{(d-7)}{2}}}{\sum_{n=-\infty}^{\infty}\left(1+\left(\frac{2 \pi n}{z}\right)^{2}\right)^{\frac{(d-5)}{2}}}
$$

From these expressions the effective exponents can be evaluated. Rather than writing the expressions down explicitly, as we will be presenting shortly the full two loop expressions we will restrict ourselves here to making some specific comments about particular dimensions.

Conveniently for $d=3, \varepsilon(z)$ and $h(z)$ are expressible in terms of elementary functions. We have explicitly

$$
\varepsilon(z)=1+\frac{z^{2} \operatorname{coth}\left(\frac{z}{2}\right)}{\sinh z+z}
$$


Similarly an explicit form for $h(z)$ is

$$
h^{-1}(z)=\frac{z \sinh \left(\frac{z}{2}\right)^{2}}{\sinh z+z}\left(\frac{1}{h\left(z_{0}\right)} \frac{\sinh z_{0}+z_{0}}{z_{0} \sinh \left(\frac{z_{0}}{2}\right)^{2}}-2 \frac{\operatorname{coth}\left(\frac{z_{0}}{2}\right)}{z_{0}}\right)+\frac{\sinh z}{\sinh z+z}
$$

We present $h$ plotted against $\ln (1 / z)$ in Figure 22 , where we choose $h_{0}=.9$ for $\ln \left(1 / z_{0}\right)=$ -7.5 . The crossover to meanfield theory is evident as $z \rightarrow \infty$.

Focusing on the universal part of the crossover by choosing $z_{0} \rightarrow \infty$, with $h\left(z_{0}\right)$ finite we find

$$
h(z)=1+\frac{z}{\sinh z}
$$

Since in the case of the two dimensional end only the $N=1$ model exhibits a standard second order phase transition we restrict our considerations to this model. (The techniques used here though can also be used to treat other values of $N$, in particular dimensional crossover in a non-linear $\sigma$ model $[47])$. We thus find

$$
\gamma_{\phi^{2}}(z)=\frac{1}{3}\left(1+\frac{z}{\sinh z}\right)
$$

As $\gamma_{\phi}=0$ to one loop so is $\eta_{e f f}$. Similarly, substituting for $h$ in (9.2) for $\nu_{e f f}$ to one loop gives

$$
\nu_{e f f}=\frac{7}{12}+\frac{z}{12 \sinh z}
$$

As $z \rightarrow \infty, \nu_{\text {eff }} \rightarrow 0.58$ and as $z \rightarrow 0, \nu_{e f f} \rightarrow 0.67$. From (9.3) our expression for $\gamma_{\text {eff }}$ is

$$
\gamma_{e f f}=\frac{7}{6}+\frac{z}{6 \sinh z}
$$

which varies between 1.17 and 1.33. To one loop $d_{\text {eff }}=3-z / \sinh z$ and varies between 3 and 2 as $z$ varies between $\infty$ and 0 . Using the scaling law (6.14) one gets $\delta_{\text {eff }}=$ $4+\frac{z}{\sinh z}$ which varies between 4 and 5 ; and from (6.15) one finds $\beta_{\text {eff }}=\frac{1}{3}-\frac{z}{6 \sinh z}$ which varies between 0.33 and 0.17 ; and finally $\alpha_{\text {eff }}=\frac{1}{6}\left(1+\frac{z}{\sinh z}\right)$. One can also determine approximations to the effective exponents by calculating them with respect to the floating fixed point. We emphasize once again that one can independently verify the scaling laws by studying the system when $T<T_{c}(L)$ (see [29]) and calculating the effective exponents directly.

In the case of $d=4$ we are not as fortunate in finding expressions in terms of elementary functions for our one loop results, however, the expressions involving the sums themselves are not too bad and are rapidly convergent. Explicitly

$$
\varepsilon(z)=1-3 \frac{\sum_{n=-\infty}^{\infty} \frac{4 \pi^{2} n^{2}}{z^{2}}\left(1+\frac{4 \pi^{2} n^{2}}{z^{2}}\right)^{-5 / 2}}{\sum_{n=-\infty}^{\infty}\left(1+\frac{4 \pi^{2} n^{2}}{z^{2}}\right)^{-3 / 2}}
$$

The one loop solution to the $\beta$ function is given by (10.3). It is now not possible to set the initial value of the coupling to a finite value and take the limit of $z_{0} \rightarrow \infty$. The logarithmic 
corrections to scaling in $d=4$ mean that the running coupling will retain a dependence on the the initial condition, i.e. the scale $\kappa$ will remain even when $L$ drops out. Since the one loop results have been presented elsewhere [27] we will not repeat them here.

\section{$\S \S 10.2$ Periodic Boundary Conditions: Two Loop Results}

From the considerations of the previous sections we can conclude that the Wilson functions are given by

$$
\begin{gathered}
\beta(h, z)=-\varepsilon(z) h+h^{2}-\frac{4}{(N+8)^{2}}\left((5 N+22) f_{1}(z)-(N+2) f_{2}(z)\right) h^{3}+O\left(h^{4}\right) \\
\gamma_{\phi^{2}}=\frac{(N+2)}{(N+8)} h-6 \frac{(N+2)}{(N+8)^{2}}\left(f_{1}(z)-\frac{1}{3} f_{2}(z)\right) h^{2}+O\left(h^{3}\right) \\
\gamma_{\phi}=2 \frac{(N+2)}{(N+8)^{2}} f_{2}(z) h^{2}+O\left(h^{3}\right)
\end{gathered}
$$

where the functions $f_{1}$ and $f_{2}$ applicable for general $d$ for this particular crossover are given in Appendix A. In Appendix B the explicit, analytic expressions for the case $d=4$, $\alpha_{1}=0, \alpha_{2}=1$ are presented. Recalling that $\nu_{e f f}=1 /\left(2-\gamma_{\phi^{2}}\right)$ and $\eta_{e f f}=\gamma_{\phi}$ we have to two loops that

$$
\nu_{e f f}=\frac{1}{2}+\frac{(N+2)}{4(N+8)} h+\frac{(N+2)\left(N+2-12 f_{1}(z)+4 f_{2}(z)\right)}{8(N+8)^{2}} h^{2}
$$

Similarly, from the scaling relation for $\gamma_{e f f}\left(\gamma_{e f f}=\nu_{e f f}\left(2-\eta_{e f f}\right)\right)$, which we have established in section 7 , we have

$$
\gamma_{e f f}=1+\frac{(N+2)}{2(N+8)} h+\frac{(N+2)\left(N+2-12 f_{1}(z)\right)}{4(N+8)^{2}} h^{2}
$$

and from the defining relation $d_{\text {eff }}=4-\varepsilon(z)-\frac{\beta(h)}{h}$ we have

$$
d_{e f f}=4-h+\frac{4}{(N+8)^{2}}\left((5 N+22) f_{1}(z)-(N+2) f_{2}(z)\right) h^{2}
$$

Using the scaling law for $\alpha_{e f f}\left(\alpha_{e f f}=2-\nu_{e f f} d_{e f f}\right)$ yields

$$
\alpha_{e f f}=\frac{(4-N)}{2(N+8)} h+\frac{\left((4-N)(N+2)-16(N+8) f_{1}(z)\right)}{4(N+8)^{2}} h^{2}
$$

The scaling law for $\beta_{\text {eff }}\left(\beta_{\text {eff }}=\frac{\nu_{e f f}}{2}\left(d_{e f f}-2+\eta_{\text {eff }}\right)\right)$ gives us

$$
\beta_{\text {eff }}=\frac{1}{2}-\frac{3}{2(N+8)} h-\frac{\left(3(N+2)-2(7 N+38) f_{1}(z)\right)}{4(N+8)^{2}} h^{2}
$$

And finally using the scaling law for $\delta_{e f f}\left(\delta_{e f f}=\left(d_{\text {eff }}+2-\eta_{\text {eff }}\right) /\left(d_{\text {eff }}-2+\eta_{\text {eff }}\right)\right)$ gives

$$
\delta_{e f f}=3+h+\frac{\left((N+8)^{2}-8(5 N+22) f_{1}(z)\right)}{2(N+8)^{2}} h^{2}
$$


Note that the functions $\varepsilon(z), f_{1}(z)$ and $f_{2}(z)$ are independent of $N$. For a three dimensional layered geometry $\left(R^{2} \times S^{1}\right)$, we find $\varepsilon(z)$ interpolates between 2 and $1, f_{1}(z)$ interpolates between 0.28 and $\frac{1}{3}$ and $f_{2}(z)$ interpolates between 0.23 and $\frac{4}{27}$ as $z$ varies from 0 to $\infty$. For a four dimensional layered geometry $\left(R^{3} \times S^{1}\right), \varepsilon(z)$ ranges monotonically from 1 , for $z=0$, to 0 for $z=\infty$, and takes the value $\frac{1}{2}$ for $z \approx 3.3$. The function $f_{1}(z)$ ranges from $\frac{1}{3}$, for $z=0$ to $\frac{1}{2}$ for $z=\infty ; f_{2}(z)$ ranges from $\frac{4}{27}$ for $z=0$ to $\frac{1}{4}$ for $z=\infty$. Thus the equations $(10.15),(10.16)$, (10.17) interpolate between those for $R^{3}$ and $R^{4}$ as $z$ ranges from 0 to $\infty$. We provide plots of $\varepsilon(z), f_{1}(z)$ and $f_{2}(z)$ in Figure 1 for the layered four dimensional geometry with periodic boundary conditions $\left(R^{3} \times S^{1}\right)$.

To explicitly implement the two loop results we resort to a Padé resummation technique. As is well known it sometimes occurs that the zero of the $\beta$-function disappears at two loops only to return at three loops. This is a property of the perturbative series and is not specific to our problem. It is equally true in the fixed dimension non-crossover case treated by Parisi. We therefore adopt Parisi's approach and use a [2,1] Padé resummation. As this agrees well with high temperature series and $\varepsilon$ expansion results there is every reason to have confidence in it as a good method of capturing the true nature of the resummed expressions. For three dimensions the two loop Padé resummed results are in excellent agreement with six loop resummed results [48], where the Callan-Symanzik equation in distinction to our homogeneous RG was used, and the best high temperature series [41]. A Borel transformation is also an option, we have not as yet followed this route. Our series are still too short to make this worthwhile. Calculations to higher orders could be implemented, and it should not be too difficult in the case of a three dimensional layered geometry to adopt the numerical techniques of [48], in conjunction with our diagramatic approach, to getting numerical curves for the expressions to a similar accuracy.

We have also resummed the series for $\gamma_{\phi^{2}}$, in terms of the Padé resummed coupling and used the scaling laws for the effective exponents. The differences between these results and those obtained when $\gamma_{\phi^{2}}$ is not resummed, and the scaling laws not used, are small for small values of $N$. We plot in Figure 10 a comparison of the one loop, two loop Padé resummed $\gamma_{\phi^{2}}$ and non-resummed $\gamma_{\phi^{2}}$ results for $\nu_{\text {eff }}$ with $N=3$. By adopting the philosophy that all the characteristic functions should be treated on an equal footing, in other words they should all be Padé resummed one retains the spherical model limit as an exact limit of the Padé resummed expressions. After Padé resumming of the characteristic functions the scaling laws should be used directly without further perturbative expansion. Our procedure is therefore to solve the Padé resummed differential equation $z \frac{\mathrm{d} h}{\mathrm{~d} z}=\beta(h, z)$ for $h(z)$ and substitute it into the Padé resummed expressions for $\gamma_{\phi^{2}}, \gamma_{\phi}$ and use the scaling laws to obtain the effective exponents. We were forced to do this numerically as the $\beta$ function equation does not have an analytic solution known to us.

An alternative procedure would have been to solve the differential equation iteratively. The one loop equation is solved first, and the solution substituted into the two loop portion of the $\beta$ function equation. The resulting differential equation is then solved. The procedure is iterated as successive loops are calculated. We have not adopted this approach, however, it would be interesting to compare the accuracy of the two procedures, this could only be reliably done at higher order than two loops.

The condition $\beta(h, z)=0$ defines from section 7 the floating fixed point, valuable in describing a system where, in terms of the usual picture of flow lines associated with the $\beta$-function, the flow lines are explicitly "time" dependent. Again note that in the $\alpha_{1}=0, \alpha_{2}=\rho_{c}^{2} \kappa^{2}$ case $z=\rho_{c} \kappa L$. The floating fixed point, as a solution of an algebraic 
and not a differential equation, is manifestly independent of $z_{0}$. In a comparison of the running and floating effective exponents, as the values of $z$ may differ by a constant scale difference, one curve may be shifted along relative to another. There is also of course some mismatch between the solution of the differential equation and the floating fixed point in the crossover region even after this shift is accounted for. This is because solving the $\beta$ function differential equation is not the same as solving the associated algebraic equation. They do, however, track one another closely (when the solution of the differential equation is dominated by a non-trivial fixed point) but not exactly. The fact that the floating fixed point is independent of initial conditions means there is no dependence on transients, hence logarithmic corrections to scaling are suppressed, however, these can be recovered by examining corrections to scaling around the floating fixed point.

The zero of $\beta(h, z)$ from (10.15) is

$$
h^{*}(z)=\varepsilon(z)+\frac{4}{(N+8)^{2}}\left((5 N+22) f_{1}(z)-(N+2) f_{2}(z)\right) \varepsilon(z)^{2}+O(\varepsilon(z))^{2}
$$

Substituting this value of $h$ into (10.16) and (10.17) gives

$$
\gamma_{\phi^{2}}^{*}(z)=\frac{(N+2)}{(N+8)} \varepsilon(z)+2 \frac{(N+2)}{(N+8)^{3}}\left((7 N+20) f_{1}(z)-(N-4) f_{2}(z)\right) \varepsilon(z)^{2}
$$

and

$$
\gamma_{\phi}^{*}(z)=2 \frac{(N+2)}{(N+8)^{2}} f_{2}(z) \varepsilon(z)^{2}
$$

These functions interpolate nicely between the two asymptotic fixed points at $z=0$ and $z=\infty$, and define finite size scaling functions around which one can develop a correction to scaling expansion, in $h-h^{*}$. The analogous [2,1] Padé resummed expression for the floating fixed-point is

$$
h^{*}(z)=\frac{\varepsilon(z)}{1-\frac{4}{(N+8)^{2}}\left((5 N+22) f_{1}(z)-(N+2) f_{2}(z)\right) \varepsilon(z)}
$$

We leave it to the reader to substitute this into the corresponding expressions for the effective exponents. Figure 11 shows a comparison of the Padé resummed floating coupling and floating fixed point for $d=4$ and periodic boundary conditions.

\section{$\S \S 10.3$ Padé Resummed Two Loop Results for Some Specific Models}

We will now write down explicit results for some models of particular interest. The two loop results we consider explicitly here are for general $d$. The explicit expressions for $f_{1}$

and $f_{2}$ can be found in Appendix A by setting $\alpha_{1}=0$ and $\alpha_{2}=1$. Specific expressions for $d=4$ are contained in Appendix B. As the expressions are rather unwieldy we choose not to exhibit them here. We should also point out here that these two loop Padé resummed results hold identically for the corresponding finite temperature relativisitic field theories. We present the direct perturbative results for ease of comparison with other methods.

For the Ising model, $N=1$, we have

$$
\beta(h, z)=-\varepsilon(z) h+h^{2}-\frac{4}{27}\left(9 f_{1}(z)-f_{2}(z)\right) h^{3}+O\left(h^{4}\right)
$$




$$
\begin{gathered}
\gamma_{\phi^{2}}(h, z)=\frac{1}{3} h-\frac{2}{9}\left(f_{1}(z)-\frac{1}{3} f_{2}(z)\right) h^{2}+O\left(h^{3}\right) \\
\gamma_{\phi}(h, z)=\frac{2}{27} f_{2}(z) h^{2}+O\left(h^{3}\right)
\end{gathered}
$$

We find the resulting effective exponents to be

$$
\begin{gathered}
\nu_{e f f}=\frac{1}{2}+\frac{1}{12} h+\frac{\left(3-12 f_{1}(z)+4 f_{2}(z)\right)}{216} h^{2} \\
\gamma_{\text {eff }}=1+\frac{1}{6} h+\frac{\left(1-4 f_{1}(z)\right)}{36} h^{2} \\
d_{\text {eff }}=4-h+\frac{4\left(9 f_{1}(z)-f_{2}(z)\right)}{27} h^{2} \\
\alpha_{e f f}(h, z)=\frac{1}{6} h+\frac{\left(1-16 f_{1}(z)\right)}{36} h^{2} \\
\beta_{\text {eff }}=\frac{1}{2}-\frac{1}{6} h+\frac{\left(10 f_{1}(z)-1\right)}{36} h^{2} \\
\delta_{\text {eff }}=3+h+\frac{\left(3-8 f_{1}(z)\right)}{6} h^{2}
\end{gathered}
$$

For $N=0$, which is related to the self avoiding random walk and polymers, we have

$$
\begin{gathered}
\beta(h, z)=-\varepsilon(z) h+h^{2}-\frac{1}{8}\left(11 f_{1}(z)-f_{2}(z)\right) h^{3}+O\left(h^{4}\right) \\
\gamma_{\phi^{2}}(h, z)=\frac{1}{16} h-\frac{3}{16}\left(f_{1}(z)-\frac{1}{3} f_{2}(z)\right) h^{2}+O\left(h^{3}\right) \\
\gamma_{\phi}(h, z)=\frac{1}{4} f_{2}(z) h^{2}+O\left(h^{3}\right)
\end{gathered}
$$

The corresponding effective exponents are then

$$
\begin{gathered}
\nu_{e f f}=\frac{1}{2}+\frac{1}{16} h+\frac{\left(1-6 f_{1}(z)+2 f_{2}(z)\right)}{128} h^{2} \\
\gamma_{e f f}=1+\frac{1}{8} h+\frac{\left(1-6 f_{1}(z)\right)}{64} h^{2} \\
d_{e f f}=4-h+\frac{\left(11 f_{1}(z)-f_{2}(z)\right)}{8} h^{2} \\
\alpha_{e f f}=\frac{1}{4} h+\frac{\left(1-16\left(f_{1}(z)\right)\right.}{32} h^{2} \\
\beta_{e f f}=\frac{1}{2}-\frac{3}{16} h+\frac{\left(38 f_{1}(z)-3\right)}{128} h^{2} \\
\delta_{e f f}=3+h+\frac{\left(4-11 f_{1}(z)\right.}{8} h^{2}
\end{gathered}
$$


For $N=-2$ which is believed to have the same two point correlation function as the Gaussian model we have

$$
\begin{gathered}
\beta(h, z)=-\varepsilon(z) h+h^{2}-\frac{4}{3} f_{1}(z) h^{3}+O\left(h^{4}\right) \\
\gamma_{\phi^{2}}(h, z)=0 \\
\gamma_{\phi}(h, z)=0
\end{gathered}
$$

As we see there is a non-trivial crossover of $\gamma_{\lambda}$, this induces a crossover in $\alpha_{e f f}, \beta_{e f f}$ and $\delta_{\text {eff }}$. The other exponents retain their mean field values, $\nu_{\text {eff }}=\frac{1}{2}$ and $\gamma_{\text {eff }}=1$. We find

$$
\begin{gathered}
d_{\text {eff }}=4-h+\frac{4 f_{1}(z)}{3} h^{2} \\
\alpha_{\text {eff }}=\frac{h}{2}-\frac{2 f_{1}(z)}{3} h^{2} \\
\beta_{\text {eff }}=\frac{1}{2}-\frac{1}{4} h+\frac{f_{1}(z)}{3} h^{2} \\
\delta_{\text {eff }}=3+h+\frac{\left(3-8 f_{1}(z)\right.}{6} h^{2}
\end{gathered}
$$

The model with $N=\infty[49]$, which is related to the spherical model and the Bose gas, yields

$$
\begin{gathered}
\beta(h, z)=-\varepsilon(z) h+h^{2} \\
\gamma_{\phi^{2}}(h, z)=h \\
\gamma_{\phi}(h, z)=0
\end{gathered}
$$

In this case the one loop expressions are exact and the effective exponents interpolate between those of the exact model in $d$ and $d-1$. We find

$$
d_{e f f}=4-h
$$

the other exponents can then be written in terms of $d_{e f f}$. Specifically

$$
\begin{aligned}
& \nu_{e f f}=\frac{1}{d_{e f f}-2} \\
& \gamma_{e f f}=\frac{2}{d_{e f f}-2}
\end{aligned}
$$

The exponent $\beta_{\text {eff }}$ does not exhibit a crossover, retaining the value $\beta_{\text {eff }}=\frac{1}{2}$ throughout the crossover.

$$
\delta_{e f f}=\frac{d_{e f f}+2}{d_{e f f}-2}
$$


The specific heat effective exponent

$$
\alpha_{e f f}=\frac{d_{e f f}-4}{d_{e f f}-2}
$$

is no longer singular for $d_{\text {eff }}<4$ and the other effective exponents do not exhibit a crossover for $d>4$, as the only fixed point of the model is at $h=0$.

The results for other physically relevant systems such as the XY-model $(N=2)$ and Heisenberg model $(N=3)$ can be easily determined from the general results above. The latter two models are of particular interest for $d=3$, but exhibit some special features which go beyond the scope of the present article, such as the Kosterlitz-Thouless transition in the former and the absence of spontaneous symmetry breaking in the latter. Our techniques can be simply adapted to the latter, in the guise of the non-linear $\sigma$ model, leading to a $\beta$ function that crosses over between the zero temperature fixed point as $z \rightarrow 0$ and the $N=3$ three dimensional critical point as $z \rightarrow \infty$, further details will be presented elsewhere.

We present our two loop results using Padé resummed Wilson functions in Figures 2 through 9. In all graphs the horizontal axis is $\ln \left(\xi_{L} / L\right)$, the different curves correspond to $N=0$ (polymers), $N=1$ (Ising model), $N=2$ (XY-model), $N=3$ (Heisenberg model) and $N=\infty$ (spherical model). The logarithmic corrections to scaling at the bulk end are clearly visible, their magnitude is as expected from four dimensional calculations. All curves are with the boundary condition $h=1$ at $\ln \left(\xi_{L} / L\right)=-20$, the value of $h$ at the initial scale parameterizes different possible crossover curves but all curves asymptote to the same form. In Figure 4 we plot $\eta_{\text {eff }}$, the exponent which governs the fall off in critical correlations at $T=T_{c}(L)$. This exponent is not a monotonic function of $N$ but attains a maximum for some value of between $N=-2$ and $N=\infty$ where it is identically zero. This is the least accurate of our exponents and the peak appears to be at $N=1$, though more accurate values for this exponent suggest it occurs at higher values, probably $N=3$. Figure 7 shows $\delta_{\text {eff }}$, note that excepting the cases $N=-2$ and $N=\infty$ the curves are very robust to changes of $N$. The crossover in the cases $N=-2$ and $N=\infty$ arises purely due to the change in $d_{e f f}$. Once again the curves are not montonic functions of $N$ there being a minimum in the vicinity of $N=3$. Figure 8 shows a plot of the effective specific heat exponent $\alpha_{e f f}$ which measures how the singular part of the free energy changes as $\Gamma$ or $T$ varies. The extra case $N=-2$ is added here, since, in the case of dimensional crossover it is distinguishable from the Gaussian model due to the fact that $\gamma_{\lambda}$ for the latter is zero whereas for the former it is non-zero being a measure of the changing effect of the leading irrelevant operator. Not only does one see the change in sign of the specific heat exponent as a function of $N$ but one also sees that the effective specific heat exponent can change sign as a function of $\xi_{L} / L$. This is quite pronounced for the XY model which starts off positive, increases then turns negative at $\xi_{L} \sim 100 \mathrm{~L}$. It would be interesting, based on the Harris criterion for the relevance or irrelevance of weak disorder, to see whether disorder could change from being irrelevant to relevant as a function of size. In Figure 9 we plot $\gamma_{\lambda}=4-d_{\text {eff }}$ which also gives information about the effective dimensionality of the system. Notice that $\gamma_{\lambda}$ is very robust to changes in $N$, varying very little across the entire range of $N,[-2, \infty]$. The asymptotic values of the effective critical exponents and associated quantities in the three dimensional regime are tabulated below. 


\begin{tabular}{|c|l|l|l|l|l|l|}
\hline \multicolumn{7}{|c|}{ Asymptotic Critical Exponents } \\
\hline $\mathrm{N}$ & $\gamma_{\phi}$ & $\gamma_{\phi^{2}}$ & $h$ & $\gamma_{\text {eff }}$ & $\nu_{\text {eff }}$ & $\alpha_{\text {eff }}$ \\
\hline-2 & $0^{*}$ & $0^{*}$ & 1.800 & $1^{*}$ & $0.5^{*}$ & $0.5^{*}$ \\
\hline-1 & 0.0200 & 0.145 & 1.820 & 1.088 & 0.550 & 0.351 \\
\hline 0 & 0.0295 & 0.277 & 1.785 & 1.175 & 0.596 & 0.211 \\
\hline 1 & 0.0329 & 0.388 & 1.732 & 1.257 & 0.639 & 0.083 \\
\hline 2 & 0.0332 & 0.479 & 1.675 & 1.330 & 0.676 & -0.029 \\
\hline 3 & 0.0322 & 0.552 & 1.621 & 1.395 & 0.709 & -0.126 \\
\hline 4 & 0.0305 & 0.611 & 1.573 & 1.451 & 0.737 & -0.211 \\
\hline$\infty$ & $0^{*}$ & $1^{*}$ & $1^{*}$ & $2^{*}$ & $1^{*}$ & $-1^{*}$ \\
\hline
\end{tabular}

* These values are exact.

All these values are in very good agreement with corresponding high temperature series and experimental results. We believe the entire crossover curves are of similar accuracy.

\section{$\S \S 10.4$ The Effect of Boundary Conditions on the Crossover.}

Let us now consider the case of other boundary conditions, more detailed results will be presented in a separate paper. Different conditions constitute an environmental probe which can be used to differentiate between systems as they fall into different crossover universality classes. We begin with Dirichlet boundary conditions, but will only be interested in "bulk" not surface physics. We will confine our considerations to one loop for simplicity. In the three dimensional layered system we are again in the fortunate position of being able to present the one loop expressions in terms of elementary functions. The floating coupling is

$$
h(y)=\left(1+\frac{\pi}{y^{2}}\right) \frac{\left(1+\frac{2 y}{\sinh y}-\frac{2 \tanh y}{y}\right)}{\left(1-\frac{\tanh y}{y}\right)}
$$

where $y=\left(z^{2}-\pi^{2}\right)^{\frac{1}{2}}$. It is necessary to consider two branches of the function $y(z)$, however, even though $y(z)$ has a branch point $h(z)$ is analytic in $z$. The floating fixed point is simply $h^{*}=\varepsilon(y)$ where

$$
\varepsilon(y)=1+\frac{3 \pi^{2}}{y^{2}}+2\left(1+\frac{\pi^{2}}{y^{2}}\right) \frac{\left(\frac{y^{2}}{\sinh ^{2} y}-\frac{\tanh y}{y}\right)}{\left(1+\frac{2 y}{\sinh 2 y}-2 \frac{\tanh y}{y}\right)}
$$

We will leave the reader to derive the corresponding one loop expressions for the effective exponents in terms of the floating fixed point. Clearly they will interpolate between exactly the same asymptotic values as for the floating coupling derived from integrating the $\beta$ function above.

In the case of anti-periodic boundary conditions the extremal values of these functions are the same, they do however differ in the crossover region. Explicitly for $d=3$, to one loop, one finds $h(y)=\left(1+\frac{\pi^{2}}{y^{2}}\right)\left(1-\frac{y}{\sinh y}\right)$. The floating fixed point is once again $\varepsilon(\bigcirc)$ where this time

$$
\varepsilon(y)=1+\frac{3 \pi^{2}}{y^{2}}-\frac{\left(\left(y^{2}+\pi^{2}\right) \tanh (y / 2)\right.}{(\sinh y-y)}
$$

Note that even though $y$ again goes through a branch point, as $z$ ranges between 0 and $\infty$, both (10.39) and (10.40) remain single valued. As was the case with Dirichlet boundary 
conditions the effective exponents crossover between the same asymptotic values, however, the effective exponents between the asymptotic values are different.

At the one loop level the expressions for general $d$, for Dirichlet boundary conditions, are

$$
\varepsilon(z)=5-d-(7-d) \frac{\sum_{n=1}^{\infty} \frac{\pi^{2}\left(n^{2}-1\right)}{z^{2}}\left(1+\frac{\pi^{2}\left(n^{2}-1\right)}{z^{2}}\right)^{\frac{d-9}{2}}}{\sum_{n=1}^{\infty}\left(1+\frac{\pi^{2}\left(n^{2}-1\right)}{z^{2}}\right)^{\frac{d-7}{2}}}
$$

and for the universal one loop floating coupling

$$
h(z)=(5-d) \frac{\sum_{n=1}^{\infty}\left(1+\frac{\pi^{2}\left(n^{2}-1\right)}{z^{2}}\right)^{\frac{(d-7)}{2}}}{\sum_{n=1}^{\infty}\left(1+\frac{\pi^{2}\left(n^{2}-1\right)}{z^{2}}\right)^{\frac{(d-5)}{2}}}
$$

For antiperiodic boundary conditions one finds

$$
\varepsilon(z)=5-d-(7-d) \frac{\sum_{n=-\infty}^{\infty} \frac{\pi^{2} n(n+1)}{z^{2}}\left(1+\frac{\pi^{2} n(n+1)}{z^{2}}\right)^{\frac{d-9}{2}}}{\sum_{n=-\infty}^{\infty}\left(1+\frac{\pi^{2} n(n+1)}{z^{2}}\right)^{\frac{d-7}{2}}}
$$

and finally for the floating coupling

$$
h(z)=(5-d) \frac{\sum_{n=-\infty}^{\infty}\left(1+\frac{\pi^{2} n(n+1)}{z^{2}}\right)^{\frac{(d-7)}{2}}}{\sum_{n=-\infty}^{\infty}\left(1+\frac{\pi^{2} n(n+1)}{z^{2}}\right)^{\frac{(d-5)}{2}}}
$$

Plots comparing the effects of different boundary conditions for a three dimensional layered Ising system can be found in graphs 12-16. The main interesting feature is in the case of Dirichlet boundary conditions, where there is a "dip" in the curves as one approaches the three dimensional regime. Thus the curves are not monotonic, the effective exponents approaching their three dimensional asymptotic values from below. The origin of this effect lies in a similar characteristic dip present in the behaviour of $\varepsilon$, and is a manifestation of the power law decay of the effects of the boundary in the case of Dirichlet boundary conditions as compared to the exponential decay for periodic boundary conditions. In the case of $d=4$ the dip in $\varepsilon$ persists and indicates that for $\frac{L}{\xi_{L}} \gg 1$ the effective dimensionality is actually above the upper critical dimension! This has some very interesting ramifications which we will return to at a later date. In the case of antiperiodic boundary conditions 
the effect is in the opposite direction to that of Dirichlet. For $d=3$ the crossover to two dimensions is completed at larger values of $L / \xi_{L}$ when compared to either Dirichlet or periodic boundary conditions. At the three dimensional end there are persistent tails relative to periodic boundary conditions. Once again these are due to the power law as opposed to exponential decay of the boundary effect. For $d=4$, in distinction to the case of Dirichlet boundary conditions, $d_{\text {eff }}$ never exceeds the upper critical dimension.

\section{$\S \S 10.5$ The Uniaxial Dipolar Ferromagnet}

Another model that falls into the same class as the considerations of this section is that of a uniaxial dipolar ferromagnet [19][20]. In this case the propagator takes the form

$$
p^{2}+\alpha_{0} \frac{p_{z}^{2}}{p^{2}}
$$

Here the environmental factor that is followed in the crossover is $\alpha_{0}$, the coupling strength of the long range dipole-dipole interaction between the spins. Once again the formal structure of the diagramatic series is the same. The diagrams differ in their dependence

on $z^{-1}=\xi_{\alpha_{0}} \alpha_{0}^{\frac{1}{2}}$ now, hence $\varepsilon(z), f_{1}(z)$ and $f_{2}(z)$ are different functions, interpolating however, in the $\alpha_{1}=0, \alpha_{2}=\rho_{c}^{2} \kappa^{2}$ prescription, between the values $1, \frac{1}{3}$ and $\frac{4}{27}$ respectively for $z=0$, to finite values close to those of the four-dimensional problem treated above. The numbers are different however, reflecting the absence of some angular integration variables. We have not as yet examined the two loop case in detail and reserve further comment till a future date. Let us therefore restrict our considerations to one loop.

We find that

$$
\varepsilon(z)=\frac{z^{2}}{1+z^{2}}
$$

The solution of the running coupling equation is given by

$$
h=\frac{1}{\sqrt{1+z^{2}}\left(h_{0}^{-1} \frac{1}{\sqrt{1+z_{0}^{2}}}+\ln \left(\frac{z_{0}\left(1+\sqrt{1+z^{2}}\right)}{z\left(1+\sqrt{1+z_{0}^{2}}\right)}\right)\right.}
$$

There is an interesting distinction between this situation and that of the dimensional crossover, in that in the case of the uniaxial problem there is a universal form for the crossover from the three dimensional fixed point to the pseudo-four dimensional fixed point. The reason for this is that the true microscopic structure of the model is three dimensional, and therefore the logarithmic growth into the UV does not continue ad infinitum. The universal part of the crossover curve can be obtained as in the case of three dimensional problems by taking the limit $z_{0} \rightarrow \infty$. We find this universal coupling is given by

$$
h(z)=\frac{1}{\sqrt{1+z^{2}} \ln \left(\frac{1+\sqrt{1+z^{2}}}{z}\right)}
$$

We present a comparison of $\nu_{\text {eff }}$ for the four dimensional dimensional crossover in a layered geometry with periodic boundary conditions with that of the uniaxial crossover in figure 23. The initial coupling for the layered geometry is $h_{0}=1$ for $\ln \left(1 / z_{0}\right)=-20$ and the curve for the uniaxial problem is the universal crossover exponent derived from (10.48). 
In both cases we plot $\nu_{e f f}=\frac{1}{2-\gamma_{\phi^{2}}}$, where $\gamma_{\phi^{2}}$ is retained to order $h$ without further expansion of the denominator. We choose this form since it is closer to the two loop results as demonstrated in Figure 10. Figure 21 clearly shows that the uniaxial crossover and that of the layered geometry are in different crossover universality classes.

As shown by Kamien and Nelson [50] recently, critical phenomena of, single component and binary mixture in directed polymers in an external field, strands of dipoles in both ferro- and electrorhealogical fluids, and flux lines in high- $T_{c}$ superconductors, are all in the same universality class as three dimensional uniaxial dipolar ferromagnets and ferroelectrics. It would be interesting to compute the effective critical exponents for the crossover behaviour in these systems based on the methodology here.

\section{$\S$ 11. CROSSOVER IN THE QUANTAL ISING MODEL}

In this section we wish to consider yet another environmental factor - $\hbar$ ! The real world is quantum mechanical, although this is not always a dominant feature governing the behaviour of systems, the macroscopic world typically being governed by classical laws. However, at low temperatures quantum effects play an important role, and typically quantum fluctuations need to be taken into account. When one is dealing with finite temperature systems there are also thermal fluctuations. An interesting question therefore, especially in the theory of phase transitions, is how does one crossover between the two types of fluctuations in a realistic system. For very low temperatures one expects quantum fluctuations to dominate, and at higher temperatures classical thermal fluctuations.

The explicit model we consider is an Ising ferromagnet in a transverse magnetic field $\Gamma$, described by the lattice Hamiltonian

$$
\mathcal{H}=-\frac{1}{2} \sum_{i j} K_{i j} S_{i}^{z} S_{j}^{z}-\Gamma \sum_{i} S_{i}^{x}
$$

where $S_{i}^{a}$ are the components of spin in the $a$ direction at lattice site $i$. The spins $S_{i}^{z}$ and $S_{j}^{x}$ do not commute and it is this non-commutation that gives the additional complications of the model over that of the usual Ising model. The model is known to have a second order transition, at a temperature $T_{c}(\Gamma)$ for $\Gamma \leq \Gamma_{c}$, while for $\Gamma>\Gamma_{c}$ no ordered phase occurs at any temperature.

As we are only interested in the critical properties of this model here we will use the LGW Hamiltonian derived by Hertz [43] using the Stratonovich-Hubbard transformation, but now a time ordered version where time ordering is with respect to imaginary times between 0 and $\beta$. The explicit Hamiltonian is

$$
\begin{aligned}
H= & \int d^{d} x \sum_{n}\left(\nabla \phi_{n} \nabla \phi_{-n}+\frac{1}{2}\left(m_{B}^{2}+g_{B}+4 \pi^{2} n^{2} T^{2}\right) \phi_{n} \phi_{-n}\right) \\
& +\frac{\lambda T}{4 !} \sum_{n_{1}, n_{2}, n_{3}, n_{4}} \delta\left(n_{1}+n_{2}+n_{3}+n_{4}\right) \phi_{n_{1}} \phi_{n_{2}} \phi_{n_{3}} \phi_{n_{4}}
\end{aligned}
$$

where we have dropped operators irrelevant in the critical region. $m_{B}^{2}+g_{B}=\Gamma-\Gamma_{c}$, where $\Gamma_{c}$ is the critical transverse field in the mean field approximation. The sum is over the Matsubara frequencies and $T=\frac{\sqrt{\alpha}}{\beta}$, where $\alpha=\frac{1}{4 \mu a^{2} \Gamma \tanh (\beta \Gamma)}$ and $\beta$ is the inverse 
temperature. We have also used $K_{q}=K_{0}-\mu a^{2} q^{2}+O\left(q^{4}\right)$ for the Fourier transform of the Ising spin-spin coupling. Observe that $\alpha$ is dimensionless, once $\beta$ and $a$ are assigned the units of length, since from the original Hamiltonian we deduce that $J, \mu$ and $\Gamma$ have the dimensions of inverse length.

In this problem the anisotropy parameter, or environmental variable, is $T$, which is essentially just the thermodynamic temperature. Note that it is entering here in a very different fashion to the dimensional crossover considered in section 10. There the temperature entered in the standard way in the quadratic term of the LGW Hamiltonian, i.e. as $T-T_{c}(L)$. We now wish to renormalize in an environmentally friendly manner. We use the conditions $(5.2-5.5)$. The condition (5.2) implies, with the multiplicative renormalization of $g$, that $g$ is proportional to $\Gamma-\Gamma_{c}(T)$, the deviation from the critical line. Note that in the renormalization used here $T$ does not renormalize, i.e. it is a non-linear scaling field. Our task now is to calculate the solution of the $\beta$ function and to subsequently compute the effective exponents using the "black box" expressions of sections 5 to 9 . The only thing we need to remember is that the environmentally friendly propagator that enters these expressions is $G(\mathbf{k}, \omega)=\frac{1}{\left(\mathbf{k}^{2}+\omega^{2}+g\right)}$ where $\omega=2 \pi n T$.

Consider first a three dimensional model. From (7.1) with $N=1$, and using the above propagator in the diagrams, one finds a floating fixed point that interpolates between the classical and quantum fixed points. The quantum fixed point in this case is found to be zero, but there exist important logarithmic corrections to scaling which are captured by the floating coupling and are visible in Figures 16-20. An examination of $d_{\text {eff }}$ reveals that it interpolates between four and three. This implies that the effects of quantum fluctuations are such as to increase the effective dimensionality of the system in the low temperature regime. The classical fixed point is $h(g=0)=1.732$. The effective exponents can be found from the floating coupling expressions of section 9. Using the two loop Padé resummed coupling and Padé resummed $\gamma_{\phi^{2}}$ for this model, the exponents $\nu_{\text {eff }}, \eta_{\text {eff }}, \gamma_{\text {eff }}$ and $\alpha_{\text {eff }}$ are plotted in Figures 16-19. Once again they are in excellent agreement at the three dimensional end with the results of [48]. We find for instance $\nu(g=0)=0.639$. The other scaling exponents can be found from the scaling exponent laws. Needless to say the expressions found agree precisely with those obtained from a direct calculation. Using the expressions in the appendices the explicit two loop Padé resummed expressions for a two dimensional Ising model in a transverse field can be found. Note that here we are deriving the effective exponents at fixed temperature, i.e. we are approaching the critical line from the $g$ direction. We could equally well have looked at exponents defined with respect to the "environmental" $T$ direction as discussed in section 5 . We will return to this in a future paper.

As far as scaling is concerned the relevant correlation length here for scaling purposes is $\xi_{T}$ the physical correlation length of the system. If we had used a temperature independent renormalization the relevant correlation length would have been the zero temperature quantum correlation length. One can write the scaling functions in universal scaling form

$$
\Gamma^{(N)}=\xi_{T}^{\frac{N d}{2}-N-d} \mathcal{F}^{(N)}\left(T \xi_{T}\right)
$$

In particular for the susceptibility $\chi^{-1}=\xi_{T}^{-2} \mathcal{F}^{(2)}\left(T \xi_{T}\right)$. In terms of effective exponents

$$
\chi^{-1}=\exp \left(\int_{1}^{g} \nu_{e f f}\left(2-\eta_{e f f}\right) \frac{d g^{\prime}}{g^{\prime}}\right)
$$

Strictly speaking the above functions are only truly universal for $d<3$. For $d=3$ the logarithmic corrections to scaling evident in the effective exponents preclude a true scaling 
form. However, the scaling form in terms of the non-linear scaling fields of section 6 is "universal" as the corrections to scaling are captured by these fields in their dependence on initial conditions. For fixed initial conditions all scaling functions are universal even in the presence of corrections to scaling.

Turning now to the two dimensional model, at one loop we can give some nice simple analytic forms. The crossover coupling in terms of $z=T \xi_{T}$ is

$$
h(z)=1+\frac{z}{\sinh (z)}
$$

One finds that $\gamma_{\phi^{2}}(z)=\frac{1}{3} h(z)$, hence the crossover effective exponents are to one loop given by

$$
\begin{gathered}
\nu_{\text {eff }}(z)=\frac{7}{12}+\frac{1}{12 \sinh (z)} \\
\gamma_{e f f}(z)=\frac{7}{6}+\frac{z}{6 \sinh (z)}
\end{gathered}
$$

The effective dimensionality $d_{e f f}=\varepsilon(\bigcirc)$ is given by

$$
\varepsilon(z)=1+\frac{z^{2} \operatorname{coth}\left(\frac{z}{2}\right)}{\sinh z+z}
$$

which once again clearly indicates a change in effective dimensionality. In this case from two up to three in the deep quantum regime. The susceptibility scaling function at one loop is simply $\chi=z^{2}$. If one wants the results parametrized in terms of $g=\Gamma-\Gamma_{c}(T)$ then one must invert the equation

$$
g(z)=\frac{z^{2}}{(\tanh (z / 2))^{1 / 3}}
$$

For the two loop results we would have to invert the two loop version of this equation.

If one compares any of the two loop or one loop expressions for effective exponents here with the corresponding expressions for a layered Ising model with periodic boundary conditions, one will find that they have exactly the same functional form. In fact, after the replacements $g \rightarrow t, T \rightarrow L^{-1}$ they are identical. Hence we would conclude that the $d-1$ dimensional Ising model in a transverse field and the $d$ dimensional layered Ising model with periodic boundary conditions lie in the same crossover universality class.

Of course it is well known that there is a mapping between these models. This was shown by Suzuki [8] using a Trotter product formula, the extra dimension being of finite size $\beta \hbar$ with periodic boundary conditions, where $\beta$ is the inverse temperature. In the above we tried not to emphasize this point for two reasons. First of all, so that we could make the link at the level of the effective exponents in the context of which we have discussed and defined crossover universality classes; and secondly, so that we could bring to light an important point about our formalism. Suppose that at a theoretical level we knew nothing about the quantum fixed point, but that experiment had told us that the critical exponents at zero temperature were very different to those at the classical fixed point. How would one go about finding a theoretical description of the other fixed point, and indeed the entire crossover? Within our environmentally friendly formalism the answer is clear: one starts at some "microscopic" energy scale $E_{m}$, one writes down a Hamiltonian for the 
"microscopic degrees of freedom", including all relevant environmental parameters, one cranks the handle of the environmentally friendly RG and sees what comes out. If there exists another fixed point which is reached via the effects of a relevant environmental parameter, then it will be seen in the environmentally friendly RG flow. If it does not exist then it will not appear. The point is we do not need to know a priori about its properties, or even of its existence, these can all be deduced from the RG. What is equally important is that one can see when one is not implementing an environmentally friendly renormalization because perturbation theory within the context of the unfriendly group will break down. This is one's pointer as to the fact that one had missed some important environment dependence.

So, we have been able to deduce effective critical exponents to what we believe to be a very high degree of accuracy for the three dimensional quantum Ising model using two loop Padé resummed perturbation theory. We also gave explicit results at one loop level and formal results at the two loop level for the two dimensional model. The results we gave above are of course also valid for non-integer dimensions. One can even extend the results given to a four dimensional quantum Ising model. Comparing with some of the past literature we find, in contrast to the claims of Lawrie [22], that the Matsubara frequencies do in fact play an important role in determining the scaling functions for dimensions $3<d<4$, thereby allowing us to distinguish this crossover from that of a uniaxial dipolar ferromagnet or of the crossover between the Wilson-Fisher and Gaussian fixed points. Obviously for a four dimensional model there is no crossover as noted in [51] in the context of the "spherical" quantum Ising model. All effects then are due to corrections to scaling, though these can be of great importance for particle physics models in the context of Kaluza Klein theory [52]. In distinction to [43] aswell we derive flow equations that are explicitly dependent on the Matsubara frequencies.

There are other quantum statistical systems whose crossover behaviour we can compute simply by reading off the environment dependence from the LGW Hamiltonian and following our black box methodology. Naturally the results presented hold identically for quantum ferroelectrics as well as quantum ferromagnets. We could also, following the work of Hertz [43], consider (in his terminology) itinerant ferromagnets, dirty itinerant ferromagnets and itinerant antiferromagnets. These will fall into different crossover universality classes since for these models the propagators are

$$
G\left(\mathbf{k}, \omega_{n}\right)=\mathbf{k}^{2}+m_{0}^{2}+\frac{\left|\omega_{n}\right|}{|\mathbf{k}|}
$$

for the itinerant ferromagnet, while for the dirty itinerant ferromagnet

$$
G\left(\mathbf{k}, \omega_{n}\right)=\mathbf{k}^{2}+m_{0}^{2}+\frac{\left|\omega_{n}\right|}{D_{0} \mathbf{k}^{2}}
$$

and for the itinerant anti-ferromagnet

$$
G\left(\mathbf{k}, \omega_{n}\right)=\mathbf{k}^{2}+m_{0}^{2}+\tau\left|\omega_{n}\right|
$$

These will all give rise to different crossover scaling functions. We would therefore consider the environmental dependence to be relevant and classify the corresponding crossovers to be in different crossover universality classes. Clearly there is a rich vein to be explored in applying our methods to the quantum-classical crossover.

\section{$\S$ 12. CONCLUSIONS AND SPECULATIONS}

In this paper we have developed in some detail our approach to field theoretic renormalization of crossovers. As is well known the independence of the bare vertex functions of 
the normalization point gives an equation which allows reparametrizations of the vertex functions in terms of new renormalized parameters, via which one tries to implement perturbation theory. We have emphasized, however, that one should be very wary since many reparametrizations may not be very useful for a perturbative analysis of the problem of interest. Such an analysis is best implemented in terms of parameters which at a particular scale describe the relevant effective degrees of freedom at that scale, in preference to degrees of freedom which are associated with some other disparate one. We therefore emphasized that the optimum choice of parameters is very sensitive to the environment, the point being that as the environment effects the fluctuations, the effective degrees of freedom are consequently environment dependent.

Our goal was to be able to quantitatively describe scale changes in systems that exhibited crossover behaviour. Scale changes in general can be fruitfully examined by analyzing the RG equation in conjunction with the equation of dimensional analysis. Combining the latter with our notion of environmentally dependent renormalized parameters gave us a powerful RG equation using which we could follow with our reparametrizations how the couplings which were natural at a given scale changed with scale, and hence how the physical correlation functions themselves changed. One might roughly think of the philosophy implemented as: 1) check out the environment you are in at a certain scale; 2) choose the appropriate parameters for that environment at that scale; 3) change scale, but only by an infinitessimal amount; 4) reparameterize to variables which are appropriate for the environment at the new scale. By so doing one obtains an RG the fixed points of which, in the space of couplings $\mathcal{M}$, are diffeomorphic to the points of scale invariance of the system. If an RG is used which is independent of a relevant environmental parameter then this will not be true. Some of the points of scale invariance will be inaccessible to an environment independent RG used alone, i.e. these points could not be seen as fixed points generated by the group with environment independent Wilson functions. In order to access them one would have to supplement the RG by extra non-perturbative information.

Sometimes environmental factors can be safely neglected, but the art is to uncover which ones. If one is interested in a system with two crossovers induced by two parameters $g_{1}$ and $g_{2}$, respectively, and one is only interested in the crossover induced by $g_{1}$, then one could renormalize in a $g_{1}$ dependent and $g_{2}$ independent way, and still capture the desired physics. Only the full $\left(g_{1}, g_{2}\right)$ dependent $\mathrm{RG}$ will capture the full crossover structure however. When something has been neglected that ought not to have been, in all cases looked at so far, the theory has very civilly informed us, via the total breakdown in perturbation theory, that something was seriously amiss. We regard this as a very general property of crossover systems. We believe that the canonical incantations: "divergences", "strong coupling" and "non-perturbative" are, more often than not, all symptomatic of the failure to take into account the changing nature of the effective degrees of freedom of a crossover system.

Within the context of an environmentally friendly RG, dependent on a generic crossover parameter $g$, we went on to examine, in a formal way to begin with, how our program would be implemented. We derived expressions for the renormalization constants and the Wilson functions to two loops, which are valid for a rather wide class of crossover problems. A particular crossover problem could be simply addressed by writing down the diagramatic components of the expressions with propagators appropriate to the crossover in question.

Further consideration of the general properties of environmentally friendly RGs led us to discover that there were generalizations of the standard scaling laws relating the effective exponents of crossovers. These effective exponents also appeared naturally in the non-linear scaling fields which were the arguments of the scaling functions characteristic of the crossover. We indicated how one could generate scaling forms in terms of non-linear 
scaling fields for the entire crossover by taking a linear scaling field associated with one fixed point, exponentiating it, and replacing the exponent with the integral of the corresponding effective exponent. In the scaling limit when all relevant crossover length scales are much bigger than the lattice spacing the effective exponents and scaling functions are universal. For cases such as crossover in a four dimensional layered system, scaling functions retain a dependence on the initial coupling. This is due to the presence of universal logarithmic corrections to scaling. There is then no universal crossover scaling curve but rather a family of such curves which can be parameterized by the possible couplings at a fixed scale. Despite the fact that thermodynamic functions do not exhibit "true" scaling behaviour, we find that the effective exponent laws are still valid, including the logarithmic corrections to scaling. The effective exponents as functions define different crossover universality classes. As in the standard non-crossover case the existence of scaling laws implies that only two of the effective exponents are independent. However, in contradistinction to the standard case an essential ingredient in our analysis was the role played by the crossover of the Wilson function for the $\phi^{4}$ operator. The fact that the leading irrelevant operator can change its degree of irrelevance has made it impossible (to date at any rate) to follow a crossover with changing upper critical dimension using $\varepsilon$ expansion techniques. In our formalism this presented no difficulty.

The crossover was naturally captured in terms of our floating $h$ coupling which interpolated in a smooth way between the asymptotic fixed points. A natural substitute for $\varepsilon, \varepsilon\left(g \xi_{g}\right)$ appeared in $\beta\left(h, g \xi_{g}\right)$, the zero of which could be expressed in terms of a floating fixed point, $h^{*}$, which could be ordered in powers of this $\varepsilon\left(g \xi_{g}\right)$. In this case floating effective exponents appeared which also obeyed the effective exponent scaling laws. The floating fixed point had the advantage of being defined with respect to an algebraic equation rather than a differential equation, and was therefore easier to compute. The difference between crossover curves defined with respect to the floating fixed point and the running coupling as a solution of the $\beta$ function equation is due to a correction to scaling which is slowly varying and non-singular throughout the entire crossover. These correction to scaling factors are calculable in our formalism. One can think of the floating effective exponents as giving a reasonably good approximation to the true effective exponents, the former missing some of the fine detail of the crossover.

We summarized the crossover in the dimension of the $\phi^{4}$ operator in terms of $d_{\text {eff }}$, which can be thought of as being a measure of the "effective dimensionality" of the system, $\gamma_{\lambda}\left(g \xi_{g}\right)=4-d_{e f f}$ being then viewed as a measure of the the deviation of the system from its upper critical dimension, and a natural generalization of the correction to scaling exponent $\omega$. In the case of finite size crossover in dimensions $d<4$ (in the absence of transients) $d_{\text {eff }}$ can be regarded as a measure of the true change in dimensionality. This interpretation of effective dimensionality was also seen to be quite natural as $d_{\text {eff }}$ appeared in the scaling laws involving the effective exponents $\beta_{e f f}, \delta_{e f f}$ and $\alpha_{e f f}$ in exactly the same place as $d$ did in the non-crossover case. $d_{e f f}^{*}=\varepsilon(\bigcirc)$ to one loop can also be thought of as a measuring the change in dimensionality.

We discussed perturbation theory in some detail showing how and why $h$ or $h^{*}$ can be used to order perturbation theory. Expansions in either $\varepsilon$ or $1 / N$ without environmentally friendly renormalization are both perturbatively useless for crossovers. Even with environmentally friendly renormalization an $\varepsilon$ is unsuitable when the upper critical dimension can changes during the crossover. Similarly $1 / N$ is equally unsuitable in cases where the number of components of the order parameter can change. The only valid choices for all the crossovers considered were $h$ and $h^{*}$. We showed that in the crossovers where these couplings could become $\sim 1$ that our methodology lent itself readily to resummation methods, giving expressions for the $[2,1]$ Padé resummed floating fixed point and $\beta$ func- 
tion equation. We emphasized that in our RG methodology it was the Wilson functions that were perturbatively computed. Scaling functions were obtained by integration of the corresponding characteristic equations. With expressions such as exp $\int\left(h+h^{2} \ldots\right)$ which result from solving the characteristic equations, it was important not to try and expand the exponentials. The job of the RG is to replace perturbation theory directly at the level of the correlation functions with perturbation theory at the level of the Wilson functions the subsequent integration (exponentiation) of which ensures that the information in the exponentials is highly non-perturbative. Just how non-perturbative is seen readily in the explicit diagrammatic expressions for the non-linear scaling fields in section 10. Relative to standard perturbation theory there is clearly a huge amount of information in such expressions. The moral was that one should include as much information as possible in the Wilson functions as this information will be "exponentiated" by the RG. What is left out will not be exponentiated and will have to be treated in direct perturbation theory.

We then shifted attention away from a generic crossover to some specific examples. We examined in detail the case of dimensional crossover. The environmental variable in this case was the finite size of a layered geometry. We found that our environmentally sensitive RG Wilson functions depended on the thickness of the layers $L$. The immediate effect of the inclusion of $L$ was that the differential generator associated with the RG now interpolated between two extremal forms, that appropriate to a bulk system in the $L / \xi_{L} \rightarrow \infty$ limit, and that associated with a reduced system in the $L / \xi_{L} \rightarrow 0$ limit. This had the desirable effect of including both extremal exponents associated with the respective points of scale invariance, which were now fixed points of this RG, in a natural way into the Wilson functions. As a consequence of this, it was easy to see that the effective exponents interpolated between those of the extremal fixed points and furthermore to verify that the effective exponent laws were valid throughout the crossover region.

In the context of specific two loop results, we faced the issue that the non-trivial fixed point of the $\beta$ function disappears at this order. We circumvented the problem by following Parisi [40], and using a $[2,1]$ Padé approximant to re-sum the series. The resulting $\beta$ function equation was integrated numerically, and the solution substituted into the resummed perturbative series for the Wilson functions. In the four to three dimensional crossover the logarithmic corrections to scaling at the four dimensional end were seen to naturally crossover to power law scaling at the three dimensional end. Asymptotic effective exponents are in excellent agreement with the Callan-Symanzik/fixed dimension results of Baker et al. [48] and also with corresponding high temperature series and experimental results $[\mathbf{4 1}]$.

A complimentary expansion which can be included in this approach is the $\frac{1}{N}$ expansion. This is an appropriate one in the case of dimensional crossover since it does not rely on the uppercritical dimension remaining fixed. This should prove useful for examining dimensional crossovers as long as the layer dimension remains larger than two. We have, therefore, included the $N=\infty$ results, in which case the results obtained interpolate between the exact spherical model results for four and three dimensions, again exhibiting the crossover from logarithmic to power law behaviour, as found by Barber and Fisher [13]. For polymer type systems $(N=0)$ we found similar good agreement with accepted results. For completeness we presented the results for the $N=-2$ model the experimental significance of which we are, however, unaware. It had the curious property of having only a crossover in the anomalous dimension of the $\phi^{4}$ operator, due to the changing upper critical dimension of the model. Perhaps some experimental model can be found in accord with this. We found specifically that the exponents $\alpha_{\text {eff }}, \beta_{\text {eff }}$ and $\delta_{\text {eff }}$ exhibit a crossover even though $\nu_{e f f}, \gamma_{e f f}$ and $\eta_{\text {eff }}$ retain their mean field values.

Boundary conditions are another natural environmental factor which affect the critical 
fluctuations of the system. We briefly compared the differences between periodic, Dirichlet and antiperiodic boundary conditions on the dimensional crossover, above the transition temperature, and sufficiently far from any boundaries. In the three cases the formal structure of the RG is identical. We concluded that the effect of periodic and antiperiodic was similar, there is an additional shift, however, of the critical temperature, in the antiperiodic case and the functions $f_{1}\left(\frac{L}{\xi_{L}}\right)$ and $f_{2}\left(\frac{L}{\xi_{L}}\right)$ are slightly different, but have the same asymptotic, large argument values. In the case of Dirichlet boundary conditions, we found, interestingly, that there is a "dip" in the crossover curves, hence they are not monotonic. The origin of this effect lay in a similar characteristic dip present in the behaviour of $\varepsilon$, and was a manifestation of the power law decay of the effects of the boundary in the case of Dirichlet boundary conditions as compared to the exponential decay for periodic boundary conditions. In the case of $d=4$ the dip in $\varepsilon$ persisted and indicated that for $\frac{L}{\xi_{L}} \gg 1$ the effective dimensionality was actually above the upper critical dimension!

The problem of crossover in uniaxial dipolar ferromagnets was similarly briefly examined. Here the environmental factor is the dipole dipole interaction, which modifies the form of the propagator. The diagramatic series is the same, however the crossover functions have a qualitative difference which persists to the quasi-four dimensional end. A true universal crossover curve exists in which all dependence on the initial coupling to dissapears, in contrast to the dimensional crossover problem in a four dimensional layered system.

We considered also an Ising model in a transverse magnetic field. For the three dimensional model we derived two loop Padé resummed expressions for the effective exponents describing the quantum to classical crossover. For the two dimensional model we derived very simple expressions for the effective exponents. The fact that the effective exponents for the three dimensional model were identical with those for a four dimensional layered Ising mdel with periodic boundary conditions showed that these two models lay in the same crossover universality class. We compared and contrasted our results with others that exist in the literature and briefly discussed how our results could easily be adapted to other quantum/classical crossovers.

Because of the generality of our approach and the ubiquitousness of crossover behaviour this work has natural extensions to many other systems. To list but a few that readily come to mind: one would like to incorporate the effect of surface Hamiltonians. One would also like to be able to include the effect of more complicated backgrounds, such as the effect of the earth's gravitational field in the case of binary fluids. A background of instantons, or quasi-particles, or vortices for more general Hamiltonians are similarly natural directions in which to extend the current analysis. The effect of these we would expect to be most naturally encapsulated in a position dependent RG (the same would be true for the application of the methodology to a generic curved space necessary for doing early universe physics). The inclusion of dynamical effects would likewise be a natural thing to do. One would like to see the crossover of dynamical scaling effective exponents as the neighbourhood of different fixed points is reached, the RG trajectory could naturally be linked to time evolution, an idea which could be very useful in black hole formation or inflation where time gets linked to scale in a very natural way. In general one would expect to have a direction, position, and time dependent RG which responded to the anisotropic and time dependent nature of a generic environment. In the neighborhood of a given fixed point, however, the universality of that fixed point should become apparent as the associated degrees of freedom begin to fluctuate critically. There is also a natural rich mathematical structure apparent in our formulation, associated with the geometry of the space of coupling constants $\mathcal{M}$, which we plan to illucidate further in a future publication. Another interesting area which caught our attention recently is that of 
microphase separation [53], which describes the critical fluctuations in diblock copolymers [54] and chemically crosslinked two-component networks [55]. Here the propagator of interest is $1 /\left(k^{2}+m^{2}+\frac{C}{k^{2}}\right)$ where $m^{2}$ is the deviation from the critical value in the Flory interaction parameter, and $C=R_{G}^{4}, R_{G}$ being the radius of gyration, in the case of diblock copolymers, and is the elastic constant in the case of crosslinked two component networks. In our black box methodology all one has to do to determine the effective critical exponents is to substitute the above propagator into the diagramatic expressions we have given.

Our approach has natural applications in a broad range of areas, from cosmology to turbulence, where RG ideas have proved useful. The generic features we have outlined will be similar. The diagramatic structure is determined from the underlying Hamiltonian/action. The appropriate renomalization should then be addressed, taking due care to incorporate the environmental factors necessary for the most efficient treatment of the processes under study. One might be led by effective Hamiltonians which give good phenomenological descriptions of the physics in different regimes. If one is sufficently insightful the RG chosen would be the one which tracked the trajectory between the microscopic theory and the effective theory. Being so insightful is of course frequently impossible. The beauty of our approach is that one does not in principle need to know the other end. The response of the theory to its environment will tell you if you monitor it sufficently closely. This in principle could be of help in theories where one does not know the appropriate degrees of freedom at one end of the crossover such as quantum gravity.

Acknowledgements: CRS was supported by a FOM fellowship and is also grateful to D.I.A.S. for financial support. DOC is grateful to the University of Utrecht for travel support. We thank Brian Dolan for his careful reading of the manuscript and helpful conversations. We have benefited from conversations variously with the following: Pierre van Baal, Edouard Brézin, Michael Fisher, Filipe Freire, Gerard 't Hooft, Bei Lok Hu, Tom Kibble, Ian Lawrie, John Lewis, Charles Nash, Mark Novotny, Lochlainn O'Raifeartaigh, Anatoly Patrick, Joe Rudnick, Dimitri Shirkov and Rafael Sorkin.

\section{Appendix A. EVAlUATING TWO LOOP DIAGRAMS IN A $d$ DIMEN- SIONAL LAYERED GEOMETRY}

The task of this section is to evaluate the diagrams entering the RG equations in the dimensional crossover problem. We first list some basic formulae that prove useful. The introduction of Feynman parameters in combining two propagators amounts to

$$
\frac{1}{D_{1}^{a_{1}} D_{2}^{a_{2}}}=\frac{\Gamma\left(a_{1}+a_{2}\right)}{\Gamma\left(a_{1}\right) \Gamma\left(a_{2}\right)} \int_{0}^{1} d x \frac{x^{a_{1}-1}(1-x)^{a_{2}-1}}{\left(x D_{1}+(1-x) D_{2}\right)^{a_{1}+a_{2}}}
$$

A second useful identity is

$$
\int \frac{d^{n} q}{(2 \pi)^{n}} \frac{1}{\left[q^{2}+\mu^{2}\right]^{\nu}}=\frac{\Gamma\left(\nu-\frac{n}{2}\right)}{\Gamma(\nu)(4 \pi)^{n / 2}} \frac{1}{\mu^{\nu-\frac{n}{2}}}
$$

We begin by evaluating the diagram $\bigcirc$. Our diagramatic notation here is that the dots on a diagram represent the location of insertions in that diagram. The insertions could arise from different sources. $\bigcirc$ for instance arises at $O(\lambda)$ in $\Gamma^{(2,1)}$ and at $O\left(\lambda^{2}\right)$ in $\Gamma^{(4)}$.

$$
\bigcirc=\frac{1}{\kappa L} \sum_{n} \int_{q} \frac{1}{\left[q^{2}+\tau+\frac{\omega(n)}{\kappa^{2} L^{2}}\right]\left[(k-q)^{2}+\tau+\frac{\omega(n)}{\kappa^{2} L^{2}}\right]}
$$


where

$$
\int_{q} f(q)=\int_{R^{d-1}} \frac{d^{d-1} q}{(2 \pi)^{d-1}} f(q)
$$

and $k$ can be composed of several external momenta depending on the form of the insertion. For periodic boundary conditions $\omega(n)=(2 \pi n)^{2}, n=0, \pm 1, \pm 2 \ldots$ We consider only $n=0$ on the external lines. On introducing a Feynman parameter, performing the integration over $q$, and taking advantage of the translation invariance of the range of this integral, $\bigcirc$ becomes

$$
\bigcirc=\frac{\Gamma\left(\frac{5-d}{2}\right)}{\kappa L(4 \pi)^{(d-1) / 2}} \sum_{n} \int_{0}^{1} d x \frac{1}{\left[x(1-x) k^{2}+\tau+\frac{\omega(n)}{\kappa^{2} L^{2}}\right]^{(5-d) / 2}}
$$

For our generic normalization point with symmetric point momentum

$$
p_{i} \cdot p_{j}=\frac{1}{4}\left(4 \delta_{i j}-1\right) \alpha_{1}
$$

(the $p_{i}$ are the external momenta entering the vertices which combine to form $k$ ), and mass point

$$
\tau=\alpha_{2}
$$

we have

$$
\bigcirc=\frac{\Gamma\left(\frac{5-d}{2}\right)}{\kappa L(4 \pi)^{(d-1) / 2}} \sum_{n} \int_{0}^{1} d x\left[x(1-x) \alpha_{1}+\alpha_{2}+\frac{\omega(n)}{\kappa^{2} L^{2}}\right]^{(d-5) / 2}
$$

Evaluating the action of $A_{4-d}=\kappa \frac{d}{d \kappa}-(4-d)$ we get

$$
A_{4-d} \bigcirc(\kappa L)=-\frac{\Gamma\left(\frac{7-d}{2}\right)}{\kappa L(4 \pi)^{(d-1) / 2}} \sum_{n} \int_{0}^{1} d x \frac{x(1-x) \alpha_{1}+\alpha_{2}}{\left[x(1-x) \alpha_{1}+\alpha_{2}+\frac{\omega(n)}{\kappa^{2} L^{2}}\right]^{(7-d) / 2}}
$$

For the symmetric point normalization conditions, and zero mass, we have $\alpha_{1}=1$ and $\alpha_{2}=0$. Thus the standard symmetric point prescription yields

$$
\bigcirc^{s p}(\kappa L)=\frac{\Gamma\left(\frac{5-d}{2}\right)}{\kappa L(4 \pi)^{(d-1) / 2}} \sum_{n} \int_{0}^{1} d x\left[x(1-x)+\frac{\omega(n)}{\kappa^{2} L^{2}}\right]^{(d-5) / 2}
$$

and

$$
A_{(4-d)} \bigcirc^{s p}(\kappa L)=-2 \frac{\Gamma\left(\frac{7-d}{2}\right)}{\kappa L(4 \pi)^{(d-1) / 2}} \sum_{n} \int_{0}^{1} d x \frac{x(1-x)}{\left[x(1-x)+\frac{\omega(n)}{\kappa^{2} L^{2}}\right]^{(7-d) / 2}}
$$

For a mass normalization condition at zero symmetric point momentum, we are working at $\alpha_{1}=0$ and $a_{2}=1$, in which case $\bigcirc$ becomes

$$
\bigcirc^{t}(\kappa L)=\frac{\Gamma\left(\frac{5-d}{2}\right)}{\kappa L(4 \pi)^{(d-1) / 2}} \sum_{n}\left[1+\frac{\omega(n)}{\kappa^{2} L^{2}}\right]^{(d-5) / 2}
$$


and

$$
A_{(4-d)} \bigcirc^{t}(\kappa L)=-2 \frac{\Gamma\left(\frac{7-d}{2}\right)}{\kappa L(4 \pi)^{(d-1) / 2}} \sum_{n}\left[1+\frac{\omega(n)}{\kappa^{2} L^{2}}\right]^{(d-7) / 2}
$$

The advantage of the mass point prescription is that there is one less Feynman parameter integral to do.

We see that $A_{(4-d)} \bigcirc$ is in fact finite when $d<5$, and from the considerations of section 4 determines the 1-loop RG equations. A useful observation is that the asymptotic limits of all of these expressions for large and small $\kappa L$ can be obtained very simply from one another. Observe first, that in the limit $\kappa L \rightarrow 0$, only the $n=0$ term contributes, thus this limit is obtained by retaining only the $n=0$ term in the above expressions. In contrast in the limit $\kappa L \rightarrow \infty$ the sum over $n$ becomes an integral, which can be thought of as another momentum integral times a factor of $\kappa L$. Thus we can account for this limit by multiplying the expression in the limit $\kappa L=0$ (obtained by retaining only the $n=0$ term) by $\kappa L$ and replacing $d-1$ by $d$. This is equivalent to viewing the $\kappa L \rightarrow 0$ limit of the $d$ dimensional layered geometry as the limit $\kappa L \rightarrow \infty$ of a $d-1$ dimensional layered geometry. The limiting expressions don't know the difference. Of course, one can obtain the asymptotic expressions by direct methods if so desired, which allows the computation of leading asymptotic corrections. For our purposes the expressions themselves and their asymptotic limits are sufficient. Using the above reasoning we see that the asymptotic limits are

$$
A_{(4-d)} \bigcirc(\kappa L)=-2 \frac{\Gamma\left(\frac{7-d}{2}\right)}{\kappa L(4 \pi)^{(d-1) / 2}} \int_{0}^{1} d x\left[x(1-x) \alpha_{1}+\alpha_{2}\right]^{(d-5) / 2}
$$

for $\kappa L \rightarrow 0$ and

$$
A_{(4-d)} \bigcirc(\kappa L)=-2 \frac{\Gamma\left(\frac{6-d}{2}\right)}{(4 \pi)^{d / 2}} \int_{0}^{1} d x\left[x(1-x) \alpha_{1}+\alpha_{2}\right]^{(d-4) / 2}
$$

for $\kappa L \rightarrow \infty$.

In the RG equations the quantity

$$
\varepsilon(\kappa L)=4-d-\kappa \frac{d}{d \kappa} \ln \left[A_{4-d} \bigcirc\right]
$$

is of interest. This expression is clearly finite and, by our rules for relating the asymptotic values, interpolates between $4-d$ and $3-d$ as $\kappa L$ ranges from 0 to $\infty$.

Next we turn to the evaluation of the "cone" diagram $\sqrt{ }$, which appears in the two loop renormalization of $\Gamma^{(4)}$ and $\Gamma^{(2,1)}$, and is given by

$$
\begin{aligned}
\mathbb{V} & =\frac{1}{(\kappa L)^{2}} \sum_{n_{1}, n_{2}} \int_{q_{1}} \int_{q_{2}} \frac{1}{\left[q_{1}^{2}+\tau+\frac{\omega\left(n_{1}\right)}{\kappa^{2} L^{2}}\right]\left[q_{2}^{2}+\tau+\frac{\omega\left(n_{2}\right)}{\kappa^{2} L^{2}}\right]} \\
& \times \frac{1}{\left[\left(k_{1}-q_{1}\right)^{2}+\tau+\frac{\omega\left(n_{1}\right)}{\kappa^{2} L^{2}}\right]\left[\left(k_{2}+q_{1}-q_{2}\right)^{2}+\tau+\frac{\omega\left(n_{12}\right)}{\kappa^{2} L^{2}}\right]}
\end{aligned}
$$

where $n_{12}=n_{1}-n_{2}$, and the diagram is evaluated with zero external discrete momenta. Note that the diagram only depends on the two momentum variables $k_{1}$ and $k_{2}$ since we must have overall momentum conservation and there are only three vertices. 
We proceed to evaluate this diagram by first combining the second and last propagators, performing the change of variables $q_{2} \rightarrow q_{2}+x\left(k_{2}+q_{1}\right)$, to obtain

$$
\begin{aligned}
\int_{q_{2}} & \frac{1}{\left[q^{2}+\tau+\frac{\omega\left(n_{2}\right)}{\kappa^{2} L^{2}}\right]\left[\left(k_{2}+q_{1}-q_{2}\right)^{2}+\tau+\frac{\omega\left(n_{12}\right)}{\kappa^{2} L^{2}}\right]} \\
& =\frac{\Gamma\left(\frac{5-d}{2}\right)}{(4 \pi)^{(d-1) / 2}} \int_{0}^{1} d x \frac{1}{\left[x(1-x)\left(k_{2}+q_{1}\right)^{2}+\tau+\frac{\omega\left(n_{1}, n_{2}, x\right)}{\kappa^{2} L^{2}}\right]}
\end{aligned}
$$

where $\omega\left(n_{1}, n_{2}, x\right)=\omega\left(n_{12}\right) x+\omega\left(n_{1}\right)(1-x)$. Next, grouping the other two propagators we obtain

$$
\begin{aligned}
& \frac{1}{\left[q_{1}^{2}+\tau+\frac{\omega\left(n_{1}\right)}{\kappa^{2} L^{2}}\right]\left[\left(k_{1}-q_{1}\right)^{2}+\tau+\frac{\omega\left(n_{1}\right)}{\kappa^{2} L^{2}}\right]} \\
& \quad=\int_{0}^{1} d z \frac{1}{\left[\left(q-z k_{1}\right)^{2}+z(1-z) k_{1}^{2}+\tau+\frac{\omega\left(n_{1}\right)}{\kappa^{2} L^{2}}\right]^{2}}
\end{aligned}
$$

Combining (A.16) and (A.17), translating the $q_{1}$ integrand, and introducing a third Feynman parameter $y$ to combine the two remaining propagators, yields

$$
\begin{aligned}
\mathbb{V}= & \frac{\Gamma\left(\frac{9-d}{2}\right)}{(\kappa L)^{2}(4 \pi)^{(d-1) / 2}} \sum_{n_{1}, n_{2}} \int_{0}^{1} d x \int_{0}^{1} d y \int_{0}^{1} d z \int_{q_{1}} \\
& \frac{[x(1-x)]^{(d-5) / 2}(1-y) y^{(3-d) / 2}}{\left[(1-y) q_{1}^{2}+y\left(k_{3}+z k_{1}+q_{1}\right)^{2}+z(1-z) k_{1}^{2}+\tau r(x, y)+\frac{\omega\left(n_{1}, n_{2}, x, y\right)}{\kappa^{2} L^{2}}\right]^{(9-d) / 2}}
\end{aligned}
$$

where $r(x, y)=\frac{y}{x(1-x)}+(1-y)$ and $\omega\left(n_{1}, n_{2}, x, y\right)=\frac{\omega\left(n_{1}, n_{2}, x\right)}{x(1-x)} y+\omega\left(n_{1}\right)(1-y)$. Finally, performing the integration over $q_{1}$ we have

$$
\checkmark)=\frac{\Gamma(5-d)}{(\kappa L)^{2}(4 \pi)^{d-1}} \sum_{n_{1}, n_{2}} \int_{0}^{1} d x \int_{0}^{1} d y \int_{0}^{1} d z \Psi_{n_{1}, n_{2}}^{4}
$$

where

$$
\Psi_{n_{1}, n_{2}}^{4}=\frac{[x(1-x)]^{(d-5) / 2}(1-y) y^{(3-d) / 2}}{\left[(1-y)\left\{y\left(z k_{1}+k_{2}\right)^{2}+z(1-z) k_{1}^{2}\right\}+\tau r(x, y)+\frac{\omega\left(n_{1}, n_{2}, x, y\right)}{\kappa^{2} L^{2}}\right]^{(5-d)}}
$$

At our generic normalization point, $k_{1}^{2}=\alpha_{1}, k_{2}^{2}=\frac{3}{4} \alpha_{1}, k_{1} k_{2}=-\frac{1}{2} \alpha_{1}$ and $\tau=\alpha_{2}$; thus we find

$$
\begin{aligned}
& \qquad \mathcal{V}^{s p}= \\
& \frac{\Gamma(5-d)}{(\kappa L)^{2}(4 \pi)^{(d-1)}} \sum_{n_{1}, n_{2}} \int_{0}^{1} d x \int_{0}^{1} d y \int_{0}^{1} d z \frac{[x(1-x)]^{(d-5) / 2}(1-y) y^{(3-d) / 2}}{\left[s(y, z) \alpha_{1}+r(x, y) \alpha_{2}+\frac{\omega\left(n_{1}, n_{2}, x, y\right)}{\kappa^{2} L^{2}}\right]^{(5-d)}}
\end{aligned}
$$


where $s(y, z)=z(1-z)\left(1-y^{2}\right)+\frac{3}{4} y(1-y)$. This gives

$$
\left.A_{2(4-d)} \downarrow\right)=-\frac{\Gamma(6-d)}{(\kappa L)^{2}(4 \pi)^{(d-1)}} \sum_{n_{1}, n_{2}} \int_{0}^{1} d x \int_{0}^{1} d y \int_{0}^{1} d z \psi_{n_{1}, n_{2}}^{4}
$$

where

$$
\psi_{n_{1}, n_{2}}^{4}=\frac{[x(1-x)]^{(d-5) / 2}(1-y) y^{(3-d) / 2}\left\{s(y, z) \alpha_{1}+r(x, y) \alpha_{2}\right\}}{\left[s(y, z) \alpha_{1}+r(x, y) \alpha_{2}+\frac{\omega\left(n_{1}, n_{2}, x, y\right)}{\kappa^{2} L^{2}}\right]^{(6-d)}}
$$

For a mass independent symmetric point normalization one simply sets $\alpha_{1}=1$ and $\alpha_{2}=0$, and for standard mass normalization at zero symmetric point momentum one sets $\alpha_{1}=0$ and $\alpha_{2}=1$

The expressions for $A_{2(4-d)} \downarrow$ still have a simple pole at $d=4$ arising from the second order pole in the preceding expression. The pole is cancelled, however, by the diagram $\bigcirc^{2}$ which also appears in the two loop renormalization of $\Gamma^{(4)}$. The expression for the combination $A_{2(4-d)}(\sqrt{ })-\frac{1}{2} \bigcirc^{2}$ ) is non singular in the limit $d \rightarrow 4$. It is clearly desireable to combine our expressions so as to obtain manifestly finite quantities. To this end we note that $\frac{1}{2} A_{2(4-d)} \bigcirc^{2}=\bigcirc A_{(4-d)} \bigcirc$ has the expression

$$
\bigcirc A_{(4-d)} \bigcirc=-\frac{2 \Gamma(6-d)}{(\kappa L)^{2}(4 \pi)^{(d-1)}} \sum_{n_{1}, n_{2}} \int_{0}^{1} d x \int_{0}^{1} d y \int_{0}^{1} d z \psi_{n_{1}, n_{2}}^{2}
$$

where

$$
\psi_{n_{1}, n_{2}}^{2}=\frac{[x(1-x)]^{(d-5) / 2} y^{(3-d) / 2}(1-y)^{(5-d) / 2}\left\{z(1-z) \alpha_{1}+r(x, y) \alpha_{2}\right\}}{\left[\tilde{s}(x, y) \alpha_{1}+r(x, y) \alpha_{2}+\frac{\tilde{\omega}\left(n_{1}, n_{2}, x, y\right)}{\kappa^{2} L^{2}}\right]^{(6-d)}}
$$

where $\tilde{s}(x, y)=y+z(1-z)(1-y)$ and $\tilde{\omega}\left(n_{1}, n_{2}, x, y\right)=\frac{\omega\left(n_{2}\right) y}{x(1-x)}+\omega\left(n_{1}\right)(1-y)$.

The two expressions for $\left.A_{2(4-d)} \cup\right)$ and $\bigcirc A_{(4-d)} \bigcirc$ are now sufficently similar that we can combine them under the Feynman parameter integrals. The resulting expression is

$$
\left.A_{2(4-d)}(\Downarrow)-\frac{1}{2} \bigcirc^{2}\right)=-\frac{2 \Gamma(6-d)}{(\kappa L)^{2}(4 \pi)^{(d-1)}} \sum_{n_{1}, n_{2}} \int_{0}^{1} d x \int_{0}^{1} d y \int_{0}^{1} d z \Phi_{n_{1}, n_{2}}^{4}
$$

where

$$
\begin{aligned}
\Phi_{n_{1}, n_{2}}^{4}= & \psi_{n_{1}, n_{2}}^{4}-\psi_{n_{1}, n_{2}}^{2}=[x(1-x)]^{(d-5) / 2} y^{(3-d) / 2} \times \\
& {\left[\frac{(1-y)\left[s \alpha_{1}+r \alpha_{2}\right]}{\left[s \alpha_{1}+r \alpha_{2}+\frac{\omega\left(n_{1}, n_{2}, x, y\right)}{\kappa^{2} L^{2}}\right]^{(6-d)}}-\frac{(1-y)^{(5-d) / 2}\left[z(1-z) \alpha_{1}+\alpha_{2}\right]}{\left[\tilde{s} \alpha_{1}+r \alpha_{2}+\frac{\tilde{\omega}\left(n_{1}, n_{2}, x, y\right)}{\kappa^{2} L^{2}}\right]^{(6-d)}}\right] }
\end{aligned}
$$


The singularities of the integrands near $y=0$ each give rise to pole contributions in the individual diagrams, but these now cancel at the level of the combined integrand and the expression is regular at $d=4$.

Now, by our rules for extracting the asymptotic values of these expressions we obtain

$$
\left.A_{2(4-d)}(\downarrow)-\frac{1}{2} \bigcirc^{2}\right)\left.\right|_{\kappa L=0}=-\frac{2 \Gamma(6-d)}{(4 \pi)^{(d-1)}} \int_{0}^{1} d x \int_{0}^{1} d y \int_{0}^{1} d z \Phi_{0,0}
$$

where

$$
\begin{aligned}
\Phi_{0,0} & =[x(1-x)]^{(d-5) / 2} y^{(3-d) / 2} \times \\
& {\left[\frac{(1-y)\left[s \alpha_{1}+r \alpha_{2}\right]}{\left[s \alpha_{1}+r \alpha_{2}\right]^{(6-d)}}-\frac{(1-y)^{(5-d) / 2}\left[z(1-z) \alpha_{1}+a_{2}\right]}{\left[\tilde{s} \alpha_{1}+r \alpha_{2}\right]^{(6-d)}}\right] }
\end{aligned}
$$

and the $\kappa L \rightarrow \infty$ limit is obtained by replacing $d-1$ by $d$ and multiplying by $\kappa^{2} L^{2}$ (one power of $\kappa L$ for each sum). Note that

$$
f_{1}=-\frac{A_{2(4-d)}\left(\mathbb{V}-\frac{1}{2} \bigcirc^{2}\right)}{\left(A_{4-d} \bigcirc\right)^{2}}
$$

is a finite expression for all values of $\kappa L$ and interpolates between $\frac{1}{3}$ and $\frac{1}{2}$ as $\kappa L$ ranges from 0 to $\infty$ for $d=4, \alpha_{1}=0$ and $\alpha_{2}=1$, and more generally it interpolates between the values for $d-1$ and $d$ as $\kappa L$ ranges from 0 to $\infty$.

Let us now consider the diagram arising due to wavefunction renormalization.

$$
\ominus=\frac{1}{\kappa^{2} L^{2}} \sum_{n_{1}, n_{2}} \int_{q_{1}} \int_{q_{2}} \frac{1}{\left[q_{1}^{2}+\tau+\frac{\omega\left(n_{1}\right)}{\kappa^{2} L^{2}}\right]} \frac{1}{\left[q_{2}+\tau+\frac{\omega\left(n_{2}\right)}{\kappa^{2} L^{2}}\right]} \frac{1}{\left[\left(k+q_{1}-q_{2}\right)^{2}+\tau+\frac{\omega\left(n_{12}\right)}{\kappa^{2} L^{2}}\right]}
$$

and again $n_{12}=n_{1}-n_{2}$. Introducing a Feynman parameter to combine the last two propagators and performing the integral over $q_{2}$ we get

$$
\begin{aligned}
\ominus & =\frac{\Gamma\left(\frac{5-d}{2}\right)}{(\kappa L)^{2}(4 \pi)^{(d-1) / 2}} \times \\
& \sum_{n_{1}, n_{2}} \int_{q_{1}} \frac{1}{\left[q_{1}^{2}+\tau+\frac{\omega\left(n_{1}\right)}{\kappa^{2} L^{2}}\right]} \int_{0}^{1} d x\left[x(1-x)\left(k+q_{1}\right)^{2}+\tau+\frac{\omega\left(n_{1}, n_{2}, x\right)}{\kappa^{2} L^{2}}\right]^{(5-d) / 2}
\end{aligned}
$$

Introducing an additional Feynman parameter and performing the integration over $q_{1}$ yields

$$
\ominus=\frac{\Gamma(4-d)}{(\kappa L)^{2}(4 \pi)^{(d-1)}} \sum_{n_{1}, n_{2}} \int_{0}^{1} d x \int_{0}^{1} d y \frac{[x(1-x)]^{(d-5) / 2} y^{(5-d) / 2}}{\left[y(1-y) k^{2}+r(x, y) \tau+\frac{\omega\left(n_{1}, n_{2}, x, y\right)}{\kappa^{2} L^{2}}\right]^{(4-d)}}
$$


We next differentiate with respect to $k^{2}$ and evaluate at our generic normalization point obtaining

$$
\phi=\frac{-\Gamma(5-d)}{(\kappa L)^{2}(4 \pi)^{(d-1)}} \sum_{n_{1}, n_{2}} \int_{0}^{1} d x \int_{0}^{1} d y \frac{[x(1-x)]^{(d-5) / 2} y^{(5-d) / 2}(1-y)}{\left[y(1-y) \alpha_{1}+r(x, y) \alpha_{2}+\frac{\omega\left(n_{1}, n_{2}, x, y\right)}{\kappa^{2} L^{2}}\right]^{(5-d)}}
$$

Finally we have

$$
\begin{aligned}
& A_{2(4-d)} \phi= \\
& \frac{2 \Gamma(6-d)}{(\kappa L)^{2}(4 \pi)^{(d-1)}} \sum_{n_{1}, n_{2}} \int_{0}^{1} d x \int_{0}^{1} d y \frac{[x(1-x)]^{(d-5) / 2} y^{(5-d) / 2}(1-y)\left\{y(1-y) \alpha_{1}+r(x, y) \alpha_{2}\right\}}{\left[y(1-y) \alpha_{1}+r(x, y) \alpha_{2}+\frac{\omega\left(n_{1}, n_{2}, x, y\right)}{\kappa^{2} L^{2}}\right]^{(6-d)}}
\end{aligned}
$$

The expression for

$$
f_{2}=\frac{A_{2(4-d)} \phi}{\left(A_{4-d} \bigcirc\right)^{2}}
$$

is therefore finite for all values of $\kappa L$. In particular in the prescription $\alpha_{1}=0, \alpha_{2}=1$ with $d=4$ we have $f_{2}$ ranging from $\frac{4}{27}$ to $\frac{1}{4}$ as $\kappa L$ goes from 0 to $\infty$.

\section{Appendix B. EVALUATION OF THE DIAGRAMS ON $R^{3} \times S^{1}$ WITH MASS RENORMALIZATION POINT}

In this appendix, working strictly on $R^{3} \times S^{1}$ we give an alternative calculation of the diagrams $\bigcirc, \mathbb{V}, \ominus$, and the resulting expressions derived from them required for the RG equations. We calculate our expressions taking advantage of the fact that the propagator in three dimensions has the simple form

$$
G(x, y)=\frac{1}{4 \pi|x-y|} e^{-m|x-y|}
$$

In our four dimensional problem, we Fourier transform on the periodic coordinate (the $S^{1}$ of $R^{3} \times S^{1}$ ) to obtain the propagator

$$
G(x, y ; n)=\frac{1}{4 \pi|x-y|} e^{-\sqrt{\left[\tau+\frac{\omega(n)}{\kappa^{2} L^{2}}\right]}|x-y|}
$$

We will evaluate the diagrams at zero external momentum and also extract a scale $\kappa$ from each of our coordinates to work with dimensionless diagrams. Finally we observe that the corresponding three dimensional diagrams are UV finite, hence the diagrams are convergent for fixed values of $n_{i}$ labeling the discrete momenta propagating in the loops. The four dimensional UV divergences arise in this case from the sum over $n_{i}$. We will therefore regulate the diagrams by treating the sums not as being sums over all integers but as sums ranging from $-N$ to $N$ where $N$ is some very large but finite cut off value. The combinations of these diagrams that enter the RG equations are strictly finite and so no restriction on the sums in this case is necessary. 
We begin with the one loop contribution

$$
\begin{aligned}
\bigcirc & =\frac{1}{\kappa L} \sum_{n} \frac{1}{V} \int d^{3} x_{1} \int d^{3} x_{2} G^{2}\left(x_{1}, x_{2} ; n\right) \\
& =\sum_{n} \int d^{3} x \frac{1}{(4 \pi)^{2}} e^{-2 \sqrt{\left[\tau+\frac{\omega(n)}{\kappa^{2} L^{2}}\right]}|x-y|}
\end{aligned}
$$

where the translation invariance of the propagator is used to cancel the volume of $R^{3}$, which we formally took to be $V$. Transforming to spherical coordinates this integral can be evaluated. We find

$$
\bigcirc=\frac{1}{8 \pi \kappa L} \sum_{n} \frac{1}{\left[\tau+\frac{\omega(n)}{\kappa^{2} L^{2}}\right]^{\frac{1}{2}}}
$$

Therefore, setting $\tau=1$, i.e. $\alpha_{2}=1$, and acting with $A_{0}$ we get

$$
A_{0} \bigcirc=-\frac{1}{8 \pi \kappa L} \sum_{n} \frac{1}{\left[1+\frac{\omega(n)}{\kappa^{2} L^{2}}\right]^{3 / 2}}
$$

The restriction on the sum over $n$ can be removed for this contribution since it does not lead to any divergence.

We next need to evaluate the diagram $\mathbb{V}$, which is given in position space by

$$
\begin{aligned}
\mathbb{V} & =\frac{1}{V} \sum_{n_{1}, n_{2}} \int d x_{1} \int d x_{2} G\left(x_{1}, x_{2} ; m_{2}+m_{12}\right)^{2} G\left(x_{2}, x_{3} ; m_{1}\right) G\left(x_{1}, x_{3} ; m_{1}\right) \\
& =\frac{1}{V(4 \pi)^{4}} \sum_{n_{1}, n_{2}} \int d^{3} x_{1} \int d^{3} x_{2} \int d^{3} x_{3} \frac{e^{-\left(m_{2}+m_{12}\right)\left|x_{1}-x_{2}\right|}}{\left|x_{1}-x_{2}\right|^{2}} \frac{e^{-m_{1}\left|x_{2}-x_{3}\right|}}{\left|x_{2}-x_{3}\right|} \frac{e^{-m_{1}\left|x_{1}-x_{3}\right|}}{\left|x_{1}-x_{3}\right|}
\end{aligned}
$$

where we have introduced the notation

$$
m_{i}=\tau+\frac{\omega(n)}{\kappa^{2} L^{2}}
$$

with $n_{12}=n_{1}+n_{2}$. Again the sums are treated as being cut off at $n=N$.

This diagram, which has the geometrical shape of a cone, can be evaluated most simply by making a judicious choice of coordinates, taking full advantage of the translational invariance of the propagators. The most convenient is to choose the origin to be at one of the vertices, not at the apex of the cone. We choose the origin at $x_{2}=0$. With this choice the integration over the location of this vertex cancels the volume factor arising in the definition of the vertex. The cone as a geometrical object in this setting is in fact a triangle since two of the propagators coincide and as such is specified by an angle and the length of the two sides. Thus if the angle between the points $x_{1}$ and $x_{3}$ is $\theta$ the graph becomes

$$
\mathcal{V}=\sum_{n_{1}, n_{2}} \frac{1}{32 \pi^{2}} \int_{0}^{\infty} d x_{1} \int_{0}^{\infty} d x_{3} x_{3} e^{-\left(m_{2}+m_{12}\right) x_{1}} e^{-m_{1} x_{3}} \int_{-1}^{1} d \mu \frac{e^{-m_{1}\left|x_{1}-x_{3}\right|}}{\left|x_{1}-x_{3}\right|}
$$


where now $\left|x_{1}-x_{3}\right|=\sqrt{x_{1}^{2}+x_{3}^{2}-2 x_{1} x_{3} \mu}$ The integral over $\mu=\cos \theta$ can be done using

$$
\int_{-1}^{1} d \mu \frac{e^{-m \sqrt{(a-b \mu)}}}{\sqrt{a-b \mu}}=\frac{2}{b m}\left(e^{-m \sqrt{a-b}}-e^{-m \sqrt{a+b}}\right)
$$

Going to the coordinates $x=\frac{1}{2}\left(x_{1}+x_{3}\right)$ and $z=\frac{x_{1}-x_{3}}{x_{1}+x_{3}}$ our expression for the diagram becomes

$$
\mathbb{V}=\sum_{n_{1}, n_{2}} \frac{1}{(4 \pi)^{2} m_{1}} \int_{0}^{\infty} d x \int_{-1}^{1} d z \frac{e^{-\left[\left(m_{2}+m_{12}\right)(1+z)+m_{1}(1-z)\right] x}}{1+z}\left[e^{-2 m_{1}|z| x}-e^{-2 m_{1} x}\right]
$$

The integral over $x$ can now be performed to get

$$
\checkmark=\sum_{n_{1}, n_{2}} \frac{1}{(4 \pi)^{2} m_{1}} \int_{-1}^{1} \frac{d z}{(1+z)}\left\{\frac{1}{M+\left(M-2 m_{1}\right) z+2 m_{1}|z|}-\frac{1}{M+2 m_{1}+\left(M-2 m_{1}\right) z}\right\}
$$

where $M=m_{1}+m_{2}+m_{12}$. This integral can be performed by dividing the range of integration up and noting that

$$
\begin{gathered}
\int_{0}^{1} \frac{d z}{(1+z)^{2}}=\frac{1}{2} \\
\int \frac{d z}{(1-z)(a+b z)}=\frac{1}{a+b} \ln \left[\frac{a+b z}{1-z}\right] \\
\int \frac{1}{(1+z)(c+d z)}=\frac{1}{c-d} \ln \left[\frac{1+z}{c+d z}\right]
\end{gathered}
$$

Care is needed with the limits as there are apparent logarithmic singularities. These however cancel. Completing the evaluation of the diagram one obtains

$$
\mathbb{V}=\frac{1}{2(4 \pi)^{2}} \sum_{n_{1}, n_{2}} \frac{1}{m_{1} M}
$$

Note that if we keep our cutoff $N$ fixed, then in the limit $\kappa L \rightarrow 0$ the expression reduces reduces for $\tau=1$ to $\mathbb{V}=1 /\left(96 \pi^{2}\right)$. Evaluating $\left.A_{0} \downarrow\right)$ as with $A_{0} \bigcirc$ by setting $\tau=1$ and then acting with $A_{0}$ we obtain

$$
A_{0}()=-\sum_{n_{1}, n_{2}} \frac{1}{32 \pi^{2} \kappa^{2} L^{2} m_{1} M}\left(\frac{1}{m_{1}^{2}}+\frac{1}{M} \frac{1}{\tilde{M}}\right)
$$

where

$$
\frac{1}{\tilde{M}}=\frac{1}{m_{1}}+\frac{1}{m_{2}}+\frac{1}{m_{12}}
$$

Similarly

$$
\frac{1}{2} A_{0}\left(\bigcirc^{2}\right)=\bigcirc A_{0} \bigcirc=-\frac{1}{(8 \pi)^{2} \kappa^{2} L^{2}} \sum_{n_{1}, n_{2}} \frac{1}{m_{1}^{3} m_{2}}
$$


We are now in a position to put the two diagrams together to evaluate $\left.A_{0}(\downarrow)-\frac{1}{2} \bigcirc^{2}\right)$. We first evaluate

$$
\left.A_{0}(\Downarrow)-\frac{1}{2} \bigcirc^{2}\right)=-\frac{1}{32 \pi^{2} \kappa^{2} L^{2}} \sum_{n_{1}, n_{2}}\left[\frac{1}{m_{1}^{3}}\left(\frac{1}{M}-\frac{1}{2 m_{2}}\right)+\frac{1}{m_{1} M^{2} \tilde{M}}\right]
$$

where there is no need to truncate the sum since this combination of diagrams is no longer UV divergent in four dimensions.

Putting the expressions together, we obtain

$$
f_{1}=\frac{2 \sum_{n_{1}, n_{2}}\left[\frac{1}{m_{1}^{3}}\left(\frac{1}{M}-\frac{1}{2 m_{2}}\right)+\frac{1}{m_{1} M^{2} \tilde{M}}\right]}{\left(\sum_{n} \frac{1}{m^{3}}\right)^{2}}
$$

The final diagram to be computed is the diagram contributing to wavefunction renormalization. We evaluate it in position space but with a momentum $k$, in the layers $R^{3}$, flowing through the diagram. Again we Fourier transform in the layers, i.e. the $S^{1}$. The diagram is therefore given by

$$
\ominus=\sum_{n_{1}, n_{2}} \frac{1}{(\kappa L)^{2}} \int d^{3} z e^{i k z} G\left(z ; m_{1}\right) G\left(z ; m_{2}\right) G\left(z ; m_{12}\right)
$$

where translation invariance of the propagator has been used to cancel the volume factor, and the sums are again regulated by a large $n$ cutoff at $N$. This still has a logarithmic divergence due to the corresponding three dimensional divergence. The quantity of interest, to us at any rate, is the contribution to wave function renormalization, which is obtained from the derivative of this with respect to $k^{2}$. It is therefore this quantity we will focus on. Using the explicit representation for the propagator and choosing the angle between the in coming momentum $k$ and the direction $z$ so that $k z=k r \mu$, where $\mu=\cos \theta$, we have

$$
\phi=\frac{1}{32 \pi^{2} \kappa^{2} L^{2}} \sum_{n_{1}, n_{2}} \int_{0}^{\infty} \frac{d r}{r} e^{-M r} \frac{d}{d k^{2}} \int_{-1}^{1} d \mu e^{i k r \mu}
$$

Noting that

$$
\int_{-1}^{1} d \mu e^{i k r \mu}=\frac{2 \sin (k r)}{k r}
$$

we have

$$
\phi=\frac{1}{32 \pi^{2} \kappa^{2} L^{2} k^{2}} \sum_{n_{1}, n_{2}} \int_{0}^{\infty} d y e^{-\frac{M}{k} y} \frac{d}{d y}\left(\frac{\sin y}{y}\right)
$$

This can easily be evaluated using integration by parts yielding

$$
\phi=\frac{1}{32 \pi^{2} \kappa^{2} L^{2} k^{2}} \sum_{n_{1} n_{2}}\left[-1+\frac{M}{k} \tan ^{-1}\left(\frac{k}{M}\right)\right]
$$


Noting that as $k \rightarrow 0$

$$
-1+\frac{M}{k} \tan ^{-1}\left(\frac{k}{M}\right)=-\frac{1}{3}\left(\frac{k}{M}\right)^{2}+\ldots
$$

Evaluating this in the limit $k \rightarrow 0$ yields

$$
\phi=-\sum_{n_{1} n_{2}} \frac{1}{96 \pi^{2} \kappa^{2} L^{2} M^{2}}
$$

Finally the contribution needed for the RG equations is

$$
A_{0} \phi=\sum_{n_{1}, n_{2}} \frac{1}{48 \pi^{2} \kappa^{2} L^{2} M^{3} \tilde{M}}
$$

The interpolating function $f_{2}=\frac{A_{2(4-d)} \phi}{\left(A_{4-d} \bigcirc\right)^{2}}$, remembering that here $d=4$ is then given by

$$
f_{2}=\frac{4}{3} \frac{\sum_{n_{1}, n_{2}} \frac{1}{M^{3} \tilde{M}}}{\left(\sum_{n} \frac{1}{m^{3}}\right)^{2}}
$$

With $\tau=1$, in the limit $\kappa L \rightarrow 0$, only the $n_{i}=0$ modes contribute, therefore $m \rightarrow 1$ $M \rightarrow 3$ and $\tilde{M} \rightarrow \frac{1}{3}$. Thus we obtain $f_{2}(0)=\frac{4}{27}$. In the other limit, $\kappa L \rightarrow \infty$, the summations become integrals and we find $f_{2}(\infty)=\frac{1}{4}$. 


\section{REFERENCES}

[1] M.E. Fisher, Rev. Mod. Phys. 46 (1974) 597.

[2] R. Frowein, J. Kötzler, B. Schaub and H.G. Schuster, Phys. Rev. B25, (1982) 4905.

[3] M.E. Fisher and D.R. Nelson, Phys. Rev. Lett. 32, (1974) 1350.

[4] I. Rhee, F.M. Gasparini and D.J. Bishop, Phys. Rev. Lett. 63, (1989) 410.

[5] R. B. Stinchcombe J. Phys. C6, (1973), 2459.

[6] K. Binder, Phase Transitions and Critical Phenomena Vol. 5B, ed.s Domb and Green (1976).

[7] M. N. Barber, in Phase Transitions and Critical Phenomena, vol.8, eds. C. Domb and J. L. Lebowitz (Academic Press, London 1983); CPSC vol.2, ed. J. L. Cardy (North Holland, 1988).

[8] M. Suzuki, Prog. Theor. Phys. 56, (1976), 1454; Stat. Phys. 18 ed S. Hess, North Holland (1993), 432.

[9] E.K. Riedel and F. Wegner, Z. Phys. 225, (1969) 195.

[10] P. Pfeuty, D. Jasnow and M.E. Fisher, Phys. Rev. B10 (1974) 2088; S. Singh and D. Jasnow, Phys. Rev. B11 (1975) 3445.

[11] T.W. Capehart and M.E. Fisher, Phys. Rev. B13, (1976) 5021.

[12] A.E. Ferdinand and M.E. Fisher, Phys. Rev. 185 (1969) 832.

[13] M. Barber and M.E. Fisher, Ann. Phys., 77 (1973) 1.

[14] E. K. Riedel and F. Wegner, Phys. Rev. B9, (1974), 294.

[15] D.J. Amit and Y.Y. Goldschmidt, Ann. Phys., 114 (1978) 356.

[16] D.R. Nelson and E. Domany, Phys. Rev. B13 (1976) 236.

[17] P. Seglar and M.E. Fisher, J. Phys. C13 (1980) 6613.

[18] Denjoe O'Connor and C.R. Stephens, Proc. Roy. Soc. A (1993) to be published.

[19] E. Frey and F. Schwabl, Phys. Rev. B, 42 (1990) 8261.

[20] C.R. Stephens, J. of Magnetism and Magnetic Materials, 104-107 (1992) 297.

[21] D. Schmeltzer, Phys. Rev. B32, 7512 (1985).

[22] I. D. Lawrie, J. Phys. C 11, (1978), 3857.

[23] A.M. Nemirovsky and K.F. Freed, Nucl. Phys. B270[FS16] (1986) 423.

[24] E. Brézin and J. Zinn-Justin, Nucl. Phys. B257 [FS14](1985) 867; J. Rudnick, H. Guo and D. Jasnow, Jour. Stat. Phys. 41 (1985) 353.

[25] H. Matsumoto, Y. Nakano and H. Umezawa, Phys. Rev. D29 (1984) 1116. 
[26] Denjoe O'Connor, C.R. Stephens and F. Freire, Class. Quan. Grav. 23 (1993) S243; F. Freire and C.R. Stephens, Zeit. Phys. C (1993) to be published; Denjoe O'Connor, C.R. Stephens and F. Freire, Mod. Phys. Lett. A25 (1993); M. van Eijck, C.R. Stephens and C.W. van Weert, "Temperature Dependence of the QCD Coupling" Utrecht/Amsterdam preprint ITFA-93-11, THU-93/08.

[27] Denjoe O'Connor and C. R. Stephens, Nucl. Phys. B360 (1991) 297; J. Phys. A25 (1992) 101; J. of Magnetism and Magnetic Materials, 104-107 (1992) 300.

[28] Denjoe O'Connor and C.R. Stephens, "Finite Size Scaling and the Renormalization Group", preprint DIAS-STP-90-26, Imperial TP/89/90/36 (1990).

[29] F. Freire, Denjoe O'Connor and C.R. Stephens, Dimensional Crossover and Finite Size Scaling Below $T_{c}$, Univ. Utrecht preprint THU 92/36, to be published in J. Stat. Phys..

[30] F. Wegner, Phase Transitions and Critical Phenomena Vol. 6, ed.s Domb and Green (1976).

[31] E. Stueckelberg and A. Peterman, Helv. Phys. Acta. 26 (1953) 499; M. Gell-Mann and F. Low, Phys. Rev. 95 (1954) 1300; N.N. Bogoliubov and D.V. Shirkov, Doklady AN SSSR 103 (1955) 203.

[32] D.V. Shirkov, RG'91, (World Scientific 1992).

[33] L. Kadanoff, Physica 2 (1966) 263; K. Wilson, Phys. Rev. 179 (1969) 1499.

[34] G. Jona-Lasinio, Phase Transitions and Critical Phenomena Vol. 6, ed.s Domb and Green (1976).

[35] K. Wilson and J. Kogut Phys. Rep. C12 (1974) 75.

[36] A.C.D. van Enter, R. Fernandez and A. Sokal, preprint.

[37] A. Polyakov, Phys. Lett. B59 (1975) 79.

[38] J.F. Nicoll, T.S. Chang and H.E. Stanley, Phys. Rev. B12 (1975) 458.

[39] Denjoe O'Connor, C.R. Stephens and B.L. Hu, Annals of Physics 190 (1989); Denjoe O'Connor and C.R. Stephens, Phys. Rev. B43 (1991) 3652.

[40] G. Parisi, J. Stat. Phys. 23 (1980) 49.

[41] J. Zinn-Justin, Quantum Field Theory and Critical Phenomena, Clarendon Press, (Oxford 1989).

[42] A. Aharony and M. E. Fisher, Phys. Rev. Lett. 45 (1980) 679; A. Aharony and G. Ahlers, Phys. Rev. Lett. 44 (1980) 782.

[43] J. A. Hertz, Phys. Rev. B14 (1976) 1165.

[44] P. Pfeuty, J. Phys. C.: Solid St. Phys. 9 (1976) 3993.

[45] L. Dolan and R. Jackiw. Phys. Rev. D9 (1974) 3320.

[46] E. Brézin, D.J. Wallace and K.G. Wilson, Phys. Rev. Lett. 29 (1972) 591.

[47] Denjoe O'Connor and C.R. Stephens, "Dimensional Crossover in the Non-linear $\sigma$ Model", Utrecht/DIAS preprint. 
[48] G.A. Baker, B.G. Nickel and D.I. Meiron, Phys. Rev. B17 (1978) 1365.

[49] Denjoe O'Connor, C.R. Stephens and A. Bray, "Dimensional Crossover in the $N \rightarrow \infty$ limit", Utrecht/DIAS preprint.

[50] R.D. Kamien and D.R. Nelson, Jou. Stat. Phys. 71 (1993) 23.

[51] I.D. Lawrie and M.E. Fisher, J. Appl. Phys. 49 (1978) 1353.

[52] Y. Kubyshin, Denjoe O'Connor and C.R. Stephens, Class. Quan. Grav. (1993) to be published.

[53] M. Benhamou, Int. Jou. Mod. Phys. A8 (1993) 2581.

[54] L. Leibler, Macromolecules 13 (1980) 1602.

[55] P.G. de Gennes, J. Phys. Lett. 40 (1979) 69. 


\section{FIGURE CAPTIONS}

Figure 1: Graph of $\varepsilon, f_{1}$ and $f_{2}$ for $d=4$ and periodic boundary conditions.

Figure 2: Two loop [2,1] Padé resummed floating coupling, h, for $d=4$ and periodic boundary conditions. $N=0$ (polymers), $N=1$ (Ising model), $N=2$ (XY-model), $N=3$ (Heisenberg model), $N=\infty$ (Spherical model) and $N=-2$ are shown.

Figure 3: Graph of two loop [2,1] Padé resummed $\nu_{\text {eff }}$ for $d=4$ and periodic boundary conditions, $N=0,1,2,3, \infty$. For $N=-2$ we have $\nu(z)=1 / 2$ identically.

Figure 4: Graph of two loop [2,1] Padé resummed $\eta_{\text {eff }}$ for $d=4$ and periodic boundary conditions, $N=0,1,2,3$. For $N=-2$ and $N=\infty, \eta(z)=0$ identically.

Figure 5: Graph of two loop [2,1] Padé resummed $\gamma_{\text {eff }}$ for $d=4$ and periodic boundary conditions, $N=0,1,2,3, \infty$. For $N=-2, \gamma(z)=1$ identically.

Figure 6: Graph of two loop [2,1] Padé resummed $\beta_{\text {eff }}$ for $d=4$ and periodic boundary conditions, $N=-2,0,1,2,3, \infty$.

Figure 7: Graph of two loop [2,1] Padé resummed $\delta_{\text {eff }}$ for $d=4$ and periodic boundary conditions, $N=-2,0,1,2,3, \infty$.

Figure 8: Graph of two loop [2,1] Padé resummed $\alpha_{\text {eff }}$ for $d=4$ and periodic boundary conditions, $N=-2,0,1,2,3, \infty$.

Figure 9: Graph of two loop [2,1] Padé resummed $\gamma_{\lambda}\left(=4-d_{\text {eff }}\right)$ for $d=4$ and periodic boundary conditions, $N=-2,0,1,2,3, \infty$.

Figure 10: Graph comparing different approximations for $\nu_{\text {eff }}$ for a four dimensional layered Heisenberg model and periodic boundary conditions. The upper graphs correspond to two loop [2,1] Padé resummed results. In the topmost curve $\gamma_{\phi^{2}}$ was resummed as well as $\beta$ and gives $\nu(\infty)=0.709$, whereas in the lower only $\beta$ was resummed and $\nu$ expanded to second order in $h$ yielding $\nu(\infty)=0.706$. The bottom two graphs are the corresponding one loop results.

Figure 11: Graph comparing two loop [2,1] Padé resummed values for the floating coupling and the floating fixed point.

Figure 12: Graph of $\nu_{\text {eff }}$ at one loop for periodic, Dirichlet and antiperiodic boundary conditions. $d=3$ and $N=1$.

Figure 13: Graph of $\alpha_{\text {eff }}$ at one loop for periodic, Dirichlet and antiperiodic boundary conditions. $d=3$ and $N=1$.

Figure 14: Graph of $\beta_{\text {eff }}$ at one loop for periodic, Dirichlet and antiperiodic boundary conditions. $d=3$ and $N=1$.

Figure 15: Graph of $d_{\text {eff }}$ at one loop for periodic, Dirichlet and antiperiodic boundary conditions. $d=3$ and $N=1$.

Figure 16: Graph of two loop [2,1] Padé resummed $\gamma_{\phi^{2}}$ and $\nu_{\text {eff }}$ for three dimensional quantal Ising model.

Figure 17: Graph of two loop [2,1] Padé resummed $\alpha_{\text {eff }}$ and $\eta_{\text {eff }}$ for three dimensional quantal Ising model. 
Figure 18: Graph of two loop [2,1] Padé resummed $\gamma_{\text {eff }}$ and $\gamma_{\lambda}$ for three dimensional quantal Ising model.

Figure 19: Graph of $\nu_{e f f}, \gamma_{\lambda}$ and $\gamma_{\phi^{2}}$ at one loop for two dimensional quantum Ising model.

Figure 20: Graph of two loop [2,1] Padé resummed $\gamma_{\text {eff }}$ for $d=4$ and periodic boundary conditions showing the effect of different initial conditions.

Figure 21: Comparison of $\nu_{\text {eff }}$ at one loop for uniaxial dipolar ferromagnet, three dimensional quantum Ising model and four dimensional layered Ising model with periodic boundary conditions.

Figure 22: The floating coupling $h$ for three dimensional layered Ising model including crossover to mean field theory. 
This figure "fig1-1.png" is available in "png" format from: http://arxiv.org/ps/hep-th/9310198v1 
This figure "fig2-1.png" is available in "png" format from: http://arxiv.org/ps/hep-th/9310198v1 
This figure "fig1-2.png" is available in "png" format from: http://arxiv.org/ps/hep-th/9310198v1 
This figure "fig2-2.png" is available in "png" format from: http://arxiv.org/ps/hep-th/9310198v1 
This figure "fig1-3.png" is available in "png" format from: http://arxiv.org/ps/hep-th/9310198v1 
This figure "fig2-3.png" is available in "png" format from: http://arxiv.org/ps/hep-th/9310198v1 
This figure "fig1-4.png" is available in "png" format from: http://arxiv.org/ps/hep-th/9310198v1 
This figure "fig2-4.png" is available in "png" format from: http://arxiv.org/ps/hep-th/9310198v1 
This figure "fig1-5.png" is available in "png" format from: http://arxiv.org/ps/hep-th/9310198v1 
This figure "fig2-5.png" is available in "png" format from: http://arxiv.org/ps/hep-th/9310198v1 
This figure "fig1-6.png" is available in "png" format from: http://arxiv.org/ps/hep-th/9310198v1 
This figure "fig2-6.png" is available in "png" format from: http://arxiv.org/ps/hep-th/9310198v1 
This figure "fig1-7.png" is available in "png" format from: http://arxiv.org/ps/hep-th/9310198v1 
This figure "fig2-7.png" is available in "png" format from: http://arxiv.org/ps/hep-th/9310198v1 
This figure "fig1-8.png" is available in "png" format from: http://arxiv.org/ps/hep-th/9310198v1 
This figure "fig2-8.png" is available in "png" format from: http://arxiv.org/ps/hep-th/9310198v1 
This figure "fig1-9.png" is available in "png" format from: http://arxiv.org/ps/hep-th/9310198v1 
This figure "fig2-9.png" is available in "png" format from: http://arxiv.org/ps/hep-th/9310198v1 
This figure "fig1-10.png" is available in "png" format from: http://arxiv.org/ps/hep-th/9310198v1 
This figure "fig2-10.png" is available in "png" format from: http://arxiv.org/ps/hep-th/9310198v1 
This figure "fig1-11.png" is available in "png" format from: http://arxiv.org/ps/hep-th/9310198v1 
This figure "fig2-11.png" is available in "png" format from: http://arxiv.org/ps/hep-th/9310198v1 